\title{
APPLYING OPTIMIZATION TECHNIQUES TO IDENTIFY MULTISTATE COLLABORATIONS FOR IMPROVING BIOPOWER ENERGY POLICY
}

\author{
A Dissertation presented to \\ the Faculty of the Graduate School \\ at the University of Missouri
}

\author{
In Partial Fulfillment \\ of the Requirements for \\ Doctoral Degree, Industrial Engineering
}

by

BAYRAM DUNDAR

Dr. Ronald G. McGarvey, Dissertation Supervisor

DECEMBER 2017 
(c) Copyright by Bayram Dundar 2017

All Rights Reserved 
The undersigned, appointed by the Dean of the Graduate School, have examined the dissertation entitled:

\title{
APPLYING OPTIMIZATION TECHNIQUES TO IDENTIFY MULTISTATE COLLABORATIONS FOR IMPROVING BIOPOWER ENERGY POLICY
}

\author{
Presented by Bayram Dundar, \\ A candidate for Doctoral Degree, \\ And hereby certify that, in their opinion, it is worthy of acceptance.
}

Dr. Ronald G. McGarvey

Dr. James Noble

Dr. Cerry Klein

Dr. Francisco X. Aguilar 


\section{DEDICATION}

This dissertation is dedicated to my wife, Ruveyda, for all her support, and love. 


\section{ACKNOWLEDGMENTS}

I would like to express my sincere gratitude all those who provided me the possibility to complete this dissertation. First, I would like to express my deepest gratitude to my advisor Dr. Ronald McGarvey. Without his guidance and continued help, this dissertation would not have been possible. Dr. McGarvey, thank you for your exceptional insight and help that has been an outstanding source of my knowledge and strength. I would like to thank my dissertation committee members: Dr.Francisco Aguilar, Dr. James Noble and Dr. Cerry Klein. I would like to thank you, Dr. Aguilar for your wise suggestions and providing support and guidance. Thank you Dr. Noble for your valuable comments to my dissertation. I want to extend my thanks to Dr. Klein for his guidance and contributions.

In addition, I would like to thank my wife, Ruveyda, who has supported me throughout entire process, both by giving me motivation and helping me persist. I will be grateful forever for your love. My thanks and appreciations also go to my family and my wife's family who have emotionally supported me and have always given me motivation and strength.

I would like to extend my gratitude to Turkish Ministry of National Education (MEB) for providing a long term financial support during my doctoral degree in the United States. Thank you for supporting Turkish students abroad to make their dreams come true. 


\section{Contents}

ACKNOWLEDGMENTS ................. ii LIST OF FIGURES .................... vii LIST OF TABLES ...................

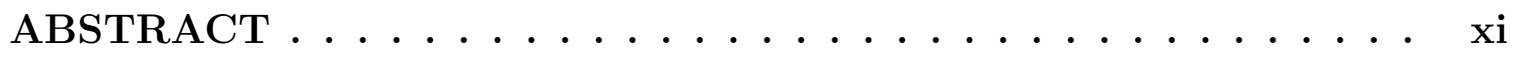
CHAPTER

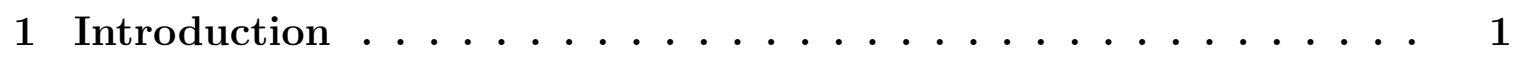

1.1 Identifying Optimal Multi-state Collaborations . . . . . . . . . . . 4

1.2 Addressing Uncertainity in Parameters . . . . . . . . . . . . . 7

1.3 Demand Response Model . . . . . . . . . . . . . . . . . . . 9

1.4 Problem Space and Contribution . . . . . . . . . . . . 10

1.5 Organization of the Dissertation . . . . . . . . . . . . . 11

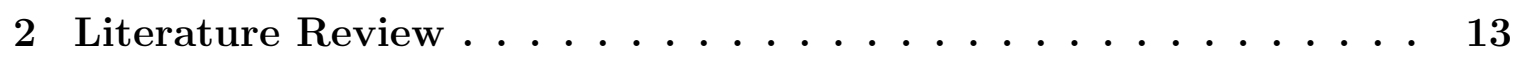

2.1 Optimization models in renewable energy generation . . . . . . . 13

2.2 Robust optimization applications in renewable energy generation . . . 16

2.3 Demand response models to account for price-demand relationship 19

3 Identifying Optimal Multi-state collaborations . . . . . . . . 22

3.1 Model Formulation . . . . . . . . . . . . . . . . 22

3.2 Data Integration . . . . . . . . . . . . . . . . 31

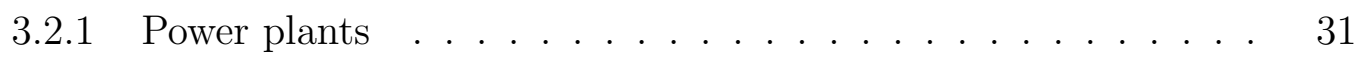


3.2 .2 Investment costs $\ldots \ldots \ldots \ldots \ldots$

3.2 .3 Biomass conversion rate . . . . . . . . . . . . . . . 34

3.2.4 Coal procurement cost and conversion rate . . . . . . . 34

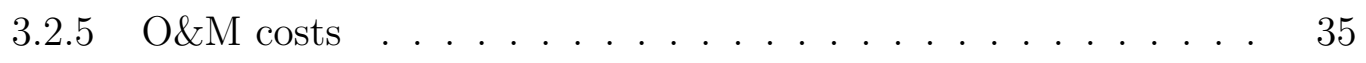

3.2 .6 Woody biomass availability $\ldots \ldots \ldots \ldots \ldots$

3.2 .7 Woody biomass procurement cost . . . . . . . . 36

3.2 .8 Electricity production requirements . . . . . . . . . 36

3.2 .9 Power plant capacity . . . . . . . . . . . . . . . . 37

3.3 Marginal Cost Integrated Approach (MCIA) $\ldots \ldots \ldots \ldots$

3.3.1 Solving the MILP to generate input data for the MCIA . . . . 41

3.4 Results and Discussion . . . . . . . . . . . . . . 42

4 Accounting for Uncertainty in Identifying Optimal Multi-state col-

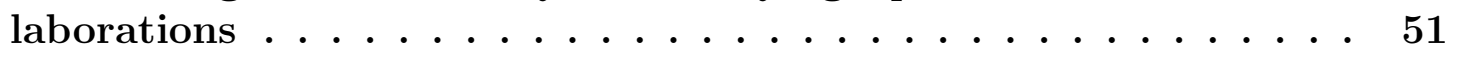

4.1 Robust Counterpart Formulations . . . . . . . . . . . . . 51

4.1.1 Robust counterpart of the objective function . . . . . . . 56

4.1.2 The uncertainty in the constraint data parameters . . . . . 59

4.1 .3 Robust counterpart of MILP . . . . . . . . . . . . . 60

4.2 Data Integration for Uncertain Data Parameters . . . . . . . . . . 64

4.2 .1 Coal electricity generation cost $\ldots \ldots \ldots \ldots \ldots$

$4.2 .2 \mathrm{CO}_{2}$ emission rate $\ldots \ldots \ldots \ldots \ldots$

4.2 .3 Boiler upgrading cost . . . . . . . . . . . . . . . . 69

4.2.4 The demand for RE generation . . . . . . . . . . . . . 69

4.3 RO - Marginal Cost Integrated Approach . . . . . . . . . . 70 
4.3.1 Generating robust marginal cost tables . . . . . . . . . . . 70

4.3.2 ROMCIA optimization model . . . . . . . . . . . . 74

4.3.3 Linearization of terms in ROMCIA model . . . . . . . . . . . 77

4.4 Results and Discussion . . . . . . . . . . . . . . . . . 79

5 A Demand Response Model for Biopower Generation to Identify Optimal Multi-State Collaborations . . . . . . . . . 85

5.1 Demand Response Model Formulation . . . . . . . . . . . . . 86

5.1.1 Estimation of the DRM parameters . . . . . . . . . 86

5.1.2 The mixed integer nonlinear objective function . . . . . . . . 90

5.1.3 Demand response model constraints . . . . . . . . . . . . . 92

5.2 A two-stage solution approach . . . . . . . . . . . . 95

5.2.1 Grid Computing . . . . . . . . . . . . . 97

5.2.2 Generation of the efficient frontiers for Multi-state plan . . . . 100

5.3 Results and Discussions . . . . . . . . . . . . . . 102

5.3.1 Demand-price relationships at a single power plant . . . . . . 102

5.3.2 Multi-state efficient frontiers . . . . . . . . . . . . . . 104

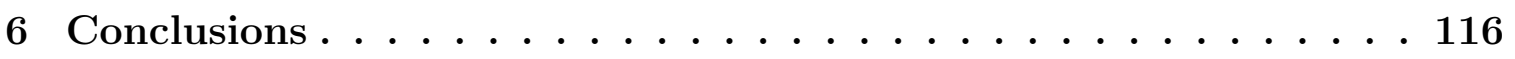

6.1 Identifying Optimal Multi-state collaborations . . . . . . . . . 116

6.2 Accounting for Uncertainty in Identifying Optimal Multi-state collaborations ........................... 118

6.3 A Demand Response Model for Biopower Generation to Identify Optimal Multi-State Collaborations . . . . . . . . . . . . . . 119

6.4 Future works . . . . . . . . . . . . . . . . . . 120

\section{APPENDIX}




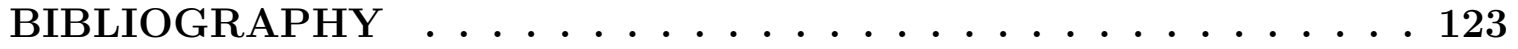

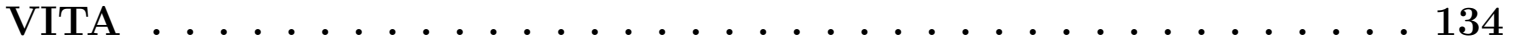




\section{List of Figures}

Figure

Page

1.1 Average percentage of state-wide electricity generated from renewable resources, and of which, from woody biomass for selected states (1990-

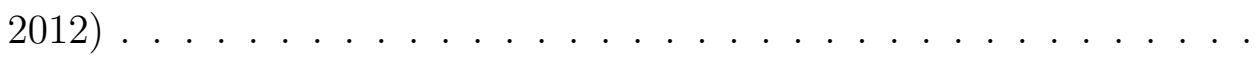

3.1 Locations of candidate power plants . . . . . . . . . . . . . 32

3.2 MCIA modeling approach . . . . . . . . . . . . . . . . . . . 39

3.3 Cost versus emission rate tradeoff, all potential partnerships including

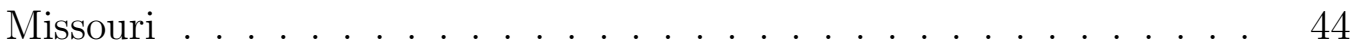

3.4 Efficient frontier of cost versus emission rate tradeoff, all potential partnerships including Missouri . . . . . . . . . . . .

3.5 Renewable energy percentage versus emission rate tradeoff, for points on efficient frontier of cost versus emission rate for all potential partnerships including Missouri . . . . . . . . . . . . . . . 50

4.1 Locations of candidate power plants . . . . . . . . . . . 65

4.2 The price of coal per ton during 2004-2015 . . . . . . . . . 67

4.3 ROMCIA modeling approach. . . . . . . . . . . . . . 71

4.4 Efficient frontier of multi-state collaboration for $0 \%$ feasibility $\ldots .80$ 
4.5 Efficient frontier of multi-state collaboration for $50 \%$ feasibility . . . . 81

4.6 Efficient frontier of multi-state collaboration for $90 \%$ feasibility . . . 82

4.7 Efficient frontiers across different feasibility probabilities . . . . . . 83

5.1 Log-transformed woody biomass consumption and its price . . . . 88

5.2 Demand response function $\mathrm{P}(\mathrm{v})$ for different radius . . . . . . . . . 89

5.3 A two-stage approach to solve single state MINLP model . . . . . . . 97

5.4 The impact of increased biomass demand on woody biomass price . . 104

5.5 The efficient frontier of multi-state collaborations for nominal model,feasibility $\Psi=0 \% \ldots \ldots \ldots \ldots \ldots$

5.6 The impact of increased biomass demand on woody biomass price for nominal model,feasibility $\Psi=0 \%$. . . . . . . . . . . . . . . 106

5.7 Detailed price of woody biomass for each state in the multi-state partnership based on nominal model, feasibility $\Psi=0 \%$. . . . . . . . 107

5.8 The efficient frontier of multi-state collaborations for feasibility $\Psi=$ $50 \% \ldots \ldots \ldots \ldots \ldots$

5.9 The impact of increased biomass demand on woody biomass price based on feasibility $\Psi=50 \%$. . . . . . . . . . . . . . . . 110

5.10 Detailed price of woody biomass for each state enter to a multi-state plan based on feasibility $\Psi=50 \%$. . . . . . . . . . . . . . . 111

5.11 The efficient frontier of multi-state collaborations for feasibility $\Psi=90 \% 112$

5.12 The impact of increased biomass demand on woody biomass price for feasibility $\Psi=90 \% \ldots \ldots . \ldots \ldots$

5.13 Detailed price of woody biomass for each state enter to a multi-state plan based on feasibility $\Psi=90 \%$. . . . . . . . . . . . . . . . . 114 
5.14 The impact of robustness on the emission rate and unit electricity generation cost . . . . . . . . . . . . . . . . . . 115 


\section{List of Tables}

Table Page

3.1 Computational run time to generate MCTs . . . . . . . . . . . . . . 42

3.2 Alternative Partnership Arrangements Generating Equal Emissions . 47

3.3 Emissions and renewable energy percentage, target and current values,

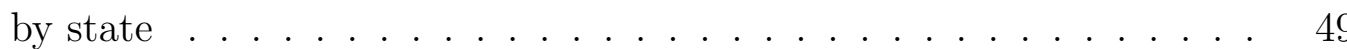

4.1 The nominal value and maximum allowable deviation (MAD) for coal price per ton and for electricity generation cost . . . . . . . . . 66

4.2 Weighted mean emission rate, by state, across power plants included in this analysis . . . . . . . . . . . . . . . . . . . . 68

4.3 Computational run time to generate each ROMCT . . . . . . . . . 72

4.4 Percentage of electricity generated from woody biomass in Missouri . 74

4.5 Contributions among partners in one multi-state collaboration . . . . 84

5.1 Demand levels, Emission Rates, and Gamma levels considered to create MCT for each state . . . . . . . . . . . . . . . . . 98

5.2 The comparison of computation time of certain number of scenarios for PA with grid computing and MILP model . . . . . . . . . . . . 100

5.3 Running times to generate single state' MCT . . . . . . . . . . . . . 101 


\title{
APPLYING OPTIMIZATION TECHNIQUES \\ TO IDENTIFY MULTISTATE COLLABORATIONS \\ FOR IMPROVING BIOPOWER ENERGY POLICY \\ BAYRAM DUNDAR
}

Dr. Ronald G. McGarvey, Dissertation Supervisor

\begin{abstract}
The US Environmental Protection Agency (EPA) proposed a rule that aims to reduce carbon emissions from US coal-fired power plants. The proposed "Clean Power Plan" specifies state-specific rate-based goals to achieve a total US carbon emission reduction of $32 \%$ below 2005 levels by 2030 . An increase in the co-firing of woody biomass with coal to generate biopower is one of the potential approaches that electricity providers could take to comply with EPA's proposed rules. We develop a mixed integer linear programming (MILP) model to identify minimum-cost approaches for reducing $\mathrm{CO}_{2}$ emissions via co-firing biomass subject to spatially-explicit biomass availability constraints. An important feature of the EPA recommendations is an allowance for states to participate in multi-state compliance strategies. We extend the MILP model to optimize within a larger geographical framework that allows states to identify minimum-cost partnerships that meet aggregated emission reduction goals. We apply the MILP model to data for five Midwestern US states to determine the role that co-firing biomass could play in achieving their EPA-proposed emission reduction targets, and find that some states can meet their renewable energy generation targets through co-firing, although co-firing alone is not sufficient to achieve any state's emission reduction targets. This MILP is extended to robust MILP model, address-
\end{abstract}


ing the uncertainties in power plant boiler installation cost, coal electricity generation cost, as well as the emission rate. We apply this robust model to a set of 18 states in the northern US to identify optimal sets of multi-state collaborations. Finally, we investigate the impact of energy policy-related regulations on biomass demand and procurement cost using econometric models. We develop a demand response model and then incorporated this into a robust mixed-integer nonlinear programming (MINLP) model. We utilize a two-stage approach to solve the resultant robust mixed integer nonlinear programming model. This model is then applied the same set of 18 states in the northern US to identify optimal sets of multi-state collaborations for different feasibility rates and emission levels. 


\section{Chapter 1}

\section{Introduction}

Carbon pollution continues to receive growing attention due to its recognition as the primary factor influencing global climate change (Kintisch 2016, Driscoll et al. 2015). The increase in worldwide $\mathrm{CO}_{2}$ levels is principally attributable to human-induced emissions, with approximately $80 \%$ of carbon emissions generated from the burning of fossil fuels (Karl 2009). Recent studies (Solomon et al. 2009, Douglas et al. 2008, Urwin \& Jordan 2008) also claim that, if the world does not reduce carbon pollution, catastrophic environmental problems will ensue. Accordingly, environmental policy decision makers across the globe have been taking actions to reduce the carbon pollution that is caused by electricity generation, transportation and other sectors that use fossil fuels. Many countries have already taken actions adopting environmental policies to reduce carbon pollution. The European Union (EU), for example, has set a target of reducing greenhouse gas emissions by $40 \%$ in 2030 compared to emissions in 1990 and increasing the share of renewable energy generation by at least $27 \%$ for 2030 (EU 2014). In 2011, the USA accounted for $16 \%$ of global $\mathrm{CO}_{2}$ emissions 
from fossil fuel combustion (Boden et al. 2015), with approximately $30 \%$ percent of US-generated carbon pollution coming from electricity generation, which was its largest source of carbon pollution (EPA 2016). To that end, governments and energy policy makers are attempting to take actions to avoid the direst effects of climate change. In 2015, the United States Environmental Protection Agency (EPA) released a proposal that aims to cut carbon emissions from existing US power plants. This proposal (EPA 2015), commonly referred to as the "Clean Power Plan", identified an overall nation-wide target of carbon emission reductions of 32\% below 2005 levels by 2030 . Moreover, each US state was provided with state-specific goals to mitigate carbon pollution from existing fossil fuel-fired electricity generation units. Instead of prescribing goals for individual power plants, the EPA proposal allowed states some flexibility to meet their specific targets, including the option to enter into multi-state partnerships to promote efficiency by reducing overall compliance costs. The new rules' ambitious goal is not only to reduce carbon pollution, but to also maintain diversity of electricity generation across renewable resources, and to increase electric system reliability, all while generating the demanded electricity at a reasonable cost. Although EPA later proposed repealing the Clean Power Plan in 2017, this decision faces an uncertain future, with years of litigation, from both proponents and opponents of the plan, the only certainty going forward (Nytimes 2017a). However, even in the absence of federal action, many U.S. states are aggressively pursuing their own plans to reduce carbon emissions from electricity, generation most notably the United States climate Alliance, including such states as California, New York, and Colorado (Nytimes 2017b).

A separate EPA report (EPA 2016) stated that 30.3\% of greenhouse gas (GHG) 
emissions in the USA were emitted by the electric power industry, making electric power the largest source of GHG emissions in the USA (followed by the transportation sector in second place). Out of the total $\mathrm{CO}_{2}$ emissions from the USA electric power industry, the majority ( $77 \%)$ are attributed to coal-fired electricity generation. Renewable resources accounted for $13 \%$ of US electricity generation in 2013, while renewable's share of electricity generation capacity was $15 \%$, according to the US Department of Energy (DOE) (DOE 2013). Over the period of 2008 to 2013, total renewable electricity generation increased by 40\% (DOE 2013). EPA (EPA 2014a) has suggested that states further expand their renewable generating capacity and, hence, reduce their carbon pollution. Although acting independently, more than half of US states have already adopted a Renewable Portfolio Standard (RPS) aiming to increase the share of electricity generated from renewable resources (DSIRE 2016).

EPA (EPA 2015) suggested four "building blocks" for strategies to reduce carbon pollution from electricity generation. These blocks were: improving heat rate at individual affected electricity generation units (EGUs); substituting more carbonintensive EGUs with less carbon-intensive EGUs; substituting generation at EGUs with expanded low- or zero-carbon emission generation; and using demand-side energy efficiency. According to EPA's proposal, woody biomass is considered to be a zeroemitting energy source for power plants. Biomass is a renewable source of energy that is derived from organic materials to generate electricity or other types of energy (Aguilar \& Garrett 2009). Woody biomass is fuel derived from wood and paper products. Woody biomass that is obtained from wood and paper by-products, as well as from logging residues and small diameter trees, can be argued to be zero- $\mathrm{CO}_{2}$ emission fuels since these are products that would otherwise be left to decompose, 
naturally releasing $\mathrm{CO}_{2}$ through decomposition (Miner et al. 2014). Other potential benefits associated with using these sources of woody biomass for energy generation include removing wildfire fuels from forests, and improving local economic conditions in areas reliant on the wood and forest industries (Dale et al. 2015, Aguilar \& Garrett 2009).

Woody biomass is both considered to be a renewable energy resource and is supported by numerous financial incentives available for energy production under RPS (Aguilar \& Saunders 2010, Ebers et al. 2016). In 2013, biomass accounted for $11 \%$ of the total US renewable electricity generation, making it the third-most utilized renewable source after hydropower and wind (DOE 2013). 67\% of this biomass generation came from wood and woody-derived fuels. One aspect of woody biomass that makes it particularly attractive for electricity generation is its ability to be co-fired with coal in many existing coal-fired power plants without prohibitively expensive renovations to the plant infrastructure.

\section{$1.1 \quad$ Identifying Optimal Multi-state Collaborations}

In this study, we consider the potential for increased use of woody biomass in cofiring to achieve both $\mathrm{CO}_{2}$ emission-reduction targets and RPS goals. Given concerns that satisfying these goals will lead to large increases in the cost of electricity (EPA 2014a), we use optimization models to identify minimum-cost approaches that generate a specified amount of electricity from power plants that can either burn coal only or co-fire woody biomass with coal, subject to constraints on allowable statewide emissions levels. Note that the EPA proposal allows states to either develop a single- 
state plan or to participate in multi-state compliance strategies. Consider Figure 1.1, which presents the percentage of electricity generated from renewable resources, and of that, the percentage generated from woody biomass, across a set of 18 states in the Northern US, over the time period 1990 - 2012 (EIA 2014a). The bars in this figure present the percentage of the state's total electricity generation produced from renewable resources (excluding hydropower). The circle markers denote the percentage contribution of woody biomass generated electricity to the state's total electricity generated from renewable energy resources (excluding hydropower).

Observe that there is significant variation in both measures across states. In Maine, for example, a significant amount of electricity was generated from renewable resources (24\%), of which the overwhelming majority (86.3\%) came from woody biomass. Iowa also generated a relatively large amount of electricity from renewable resources (11\%), but essentially none of this from woody biomass. Ohio generated relatively little energy from renewable resources (less than 1\%), but more than onehalf of this came from woody biomass. Missouri, meanwhile, generated less than $1 \%$ of its electricity from renewable resources, of which only $0.1 \%$ came from woody biomass. Consider, for example, five states in the US midwest region: Illinois (IL), Iowa (IA), Minnesota (MN), Missouri (MO), and Wisconsin (WI). We consider this set of states, in Chapter 3 in order to investigate the potential benefits that states can identify through cooperation. Despite the fact that most of these states have significant amounts of woody biomass resources, the proportion of renewable electricity generated by each state is relatively small. These five states have a relatively low current installed capacity for biomass electricity (DOE 2013), and thus are potentially good candidates for expanded production of electricity from co-firing. 


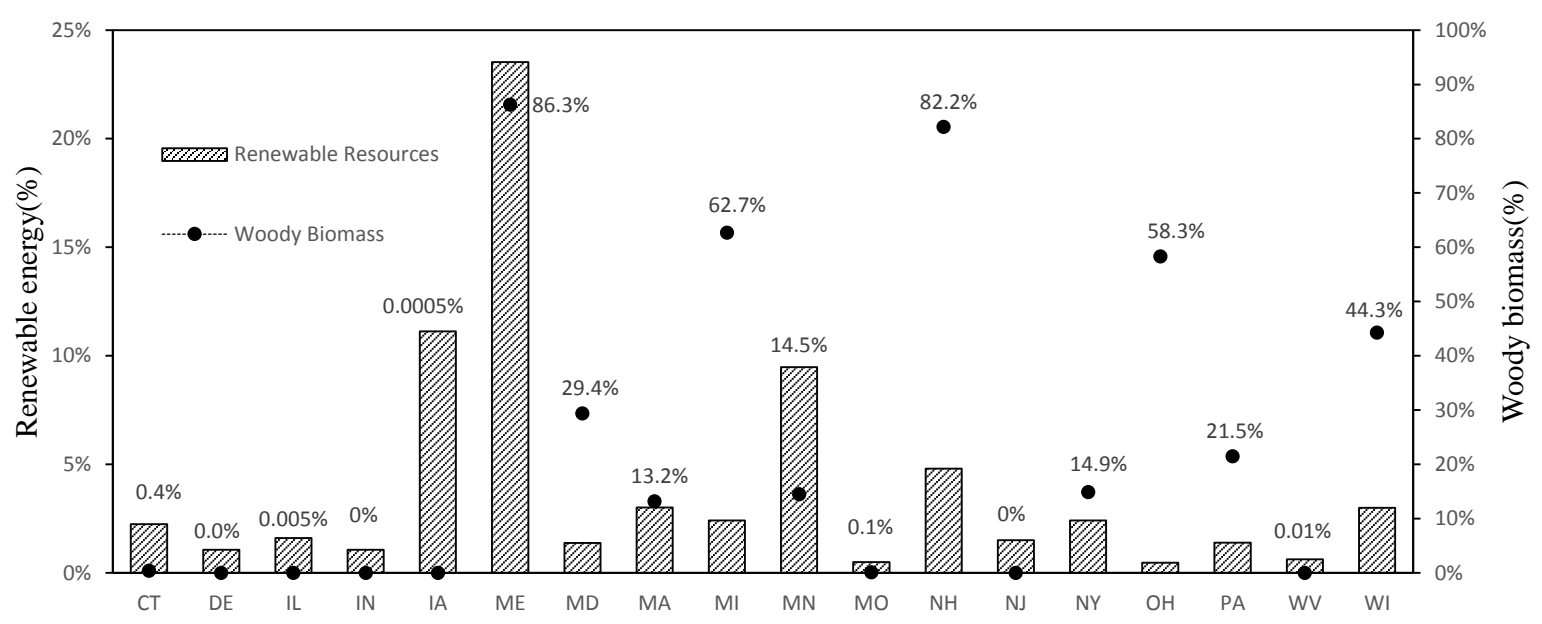

Figure 1.1: Average percentage of state-wide electricity generated from renewable resources, and of which, from woody biomass for selected states (1990-2012)

Even in states that currently generate little electricity from woody biomass, there may be the potential for increased generation from this renewable resource. Goerndt et al. (2012) utilized the US Department of Agriculture (USDA) Forest Service Forest Inventory database to determine the total woody biomass available from logging residues (slash, by-products) and small diameter trees within a $30 \mathrm{~km}, 60 \mathrm{~km}$, and $90 \mathrm{~km}$ radius of power plants within this 18-state region. This available biomass was computed such that any biomass used for energy would not be detrimental to existing standing biomass levels, and was net of woody biomass growth, removals and mortality, and set not to increase annual biomass growth. The primary reason that co-firing is not utilized to a greater extent is the relative cost-efficiency of coal, due to its high energy density and low cost, relative to woody biomass. However, should emissions reductions or minimum RPS standards be required in the future, biomass could become an attractive option for reducing emissions at existing power plants.

The primary objective of this study is to identify electricity generation approaches that reduce $\mathrm{CO}_{2}$ emission levels through co-firing woody biomass at minimum total 
cost. In order to solve this multi-state model, we initially solve a series of individualstate models for each state, and then propose a marginal cost integrated approach (MCIA) model to identify minimum-cost multi-state compliance strategies. The MCIA model is applied over the time period 2022-2030. We use the MCIA model to address the following questions:

- To what extent can the use of woody biomass in electricity generation support RPS targets? Is there sufficient wood energy feedstock located sufficiently close to existing power plants?

- Are existing power plants good candidates for co-firing woody biomass? If not, what facility upgrades would be required?

- What are the most cost-efficient approaches to attain increases in renewable energy and reductions in $\mathrm{CO}_{2}$ emissions?

- How should states approach these goals? Individually or in collaborative multistate plans?

\subsection{Addressing Uncertainity in Parameters}

An important characteristic of the 2015 EPA proposal is its allowance for states to comply with emission reduction targets either cooperatively, in multi-state partnerships, or individually. In Chapter 3, we present an optimization model for identifying multi-state partnerships that achieve aggregate emission reduction levels for electricity generation at minimum total cost. However, in this study, we utilized deterministic optimization models, and did not account for uncertainty in data parameters. This is 
a potentially-limiting feature, since many of the data that are relevant to identifying and evaluating such multi-state partnerships are subject to considerable variation. For example, historical data show considerable variation across time in the coal procurement cost (EIA 2013) and in individual power plants' emission rates (EPA 2014b). Other important parameters, such as the cost of upgrading power plant facilities to accommodate increased use of woody biomass, are uncertain due to differences across power plants and the limited set of plants that have performed such upgrades.

Therefore, in Chapter 4, we will expand this research to identify the impact of uncertainty on multi-state partnerships designed to reduce aggregate emission levels through the increased co-firing of woody biomass. A set of models, based on robust optimization (RO) concepts, will be developed and tested, to identify an efficient frontier of strategies that achieve desired emission reduction levels at a user-specified level of confidence, while reducing the potential exposure to cost increases due to data uncertainty.

The major research questions, in addition to questions in Chapter 3, to be addressed in Chapter 4 are:

- Based on the potentially-available woody biomass resource, is it possible, via cofiring, to reduce carbon pollution from EGUs and achieve RPS targets under different uncertainty levels?

- To what extent can multi-state partnerships increase these environmental benefits, at a given level of generation cost, for individual states for different uncertainty levels?

- How would uncertainty in facility upgrading cost, coal electricity generation cost, 
and plant emission rates impact the decision making?

\subsection{Demand Response Model}

Energy policy-related regulations, the cost of other types of energy resources and the increasing demand of biomass could impact the price of biomass. As mentioned earlier, based on the Clean Power plan proposal, EPA could mandate the states to generate more energy from renewable energy resources and to mitigate carbon pollution. Galik et al. (2009) investigated the availability of woody biomass and potential supply cost of woody biomass across three southern states. They found that the increasing supply of biomass could dramatically impact the resource pricing. Therefore,

in Chapter 5, we will extend our robust optimization model in order to investigate the relationship between the biomass price and demand. The main contribution of this chapter is the development of an econometric model to capture the relationship between the price of woody biomass and demand for woody biomass, and the incorporation of this model into the optimization models of Chapter 4. Thus, the following research questions will be addressed in Chapter 5 addition to the questions addressed in the previous chapters:

- How would increased demand for wood energy feedstocks impact procurement costs at a localized plant level?

- How would the efficient frontiers for multi-state partnerships be formed for the different feasibility rates?

- How would emission reduction affect the woody biomass aggregated price based on 
the efficient frontiers under different feasibility rates?

\subsection{Problem Space and Contribution}

Woody biomass has been an attractive fuel for renewable energy providing a substitute for fossil fuels. Woody biomass is used in the generation of biofuels, such as ethanol, bio-oil, and biodiesel, as well as in the generation of biopower, in the form of heat, steam, and electricity from power plants. Optimization tools have been extensively used in the harvesting, transportation, and processing activities for woody biomass, to make it an economically feasible energy source. Moreover, optimization tools have also been used in the production of biomass-fueled biofuels and biopower generation. Cambero \& Sowlati (2014) broadly reviewed the literature in the area of optimization of forest woody biomass supply chains from the economic, social and environmental perspectives. As Cambero \& Sowlati (2014) discussed, limited studies have been done in woody biomass supply chains examining both economic and environmental considerations. The authors also indicated that there is insufficient study that considers uncertainty in the woody biomass supply chain. To the best of our knowledge, there is no study that takes into account uncertainty in cofiring of woody biomass and coal. Therefore, in this study, we approach biopower generation from state partnership perspective by considering economic and environmental concerns. We employ optimization models focused on electricity generation from woody biomass via cofiring coal in existing power plants. We also developed efficient frontiers that show the tradeoff between carbon reduction and unit electricity generation cost for

multistate partnerships while considering uncertainty. One of the novel contribution 
of this study is providing the efficient frontiers as decision support systems so that energy policymakers can make the best decision based on their individual valuations of cost versus emission reductions. This study is also novel in terms of incorporating a demand response model into an optimization model with respect to energy policy issues, which investigates woody biomass price and demand relationship in cofiring from both a plant-based and broader states-wide perspective.

\subsection{Organization of the Dissertation}

The remainder of this dissertation is structured as follows. Chapter 2 presents a review of the application of operations research models to renewable energy production. Our initial models appear in Chapter 5. Section 3.1 presents detailed information about the initial, non-robust single-state model and its components. Section 3.2 briefly explains how we integrated the model's data parameters. Section 3.3 is devoted to the development of an MCIA solution approach for reducing the computational run time of our model. Section 3.4 provides detailed results of the application of this model to a five-state example, demonstrating how this modeling approach can be utilized to determine optimal partnering strategies for one state, namely, Missouri.

Chapter 4 presents an extension of our model that utilizes robust optimization to identify multi-state collaborations for reducing $\mathrm{CO}_{2}$ emissions by co-firing biomass in EGUs while accounting for uncertainties. Section 4.1 provides a detailed explanation of our single-state robust model. Section 4.2 briefly explains how we determine the nominal and range values of uncertain parameters. Section 4.3 introduces a Robust

Optimization MCIA(ROMCIA) solution approach to reduce computational run time 
for the multi-state robust model. Section 4.4 provides computational results of an application of the ROMCIA model to an 18-state example

In Chapter 5, we employ a demand response model, in which elasticity effects are added to capture the relationship between demand for biomass and biomass procurement costs. Section 5.1 presents the process of developing the demand response model and how to incorporate it into a robust mixed integer nonlinear program$\operatorname{ming}($ MINLP) model. Section 5.2 provides the procedures applied to solve this robust MINLP model, and computational experience. Section 5.3 discusses the results of applying this robust MINLP containing a demand response model to the same 18 state example.

Finally, we conclude in this dissertation by summarizing our key findings and discussing possible extensions to this work in Chapter 6 . 


\section{Chapter 2}

\section{Literature Review}

\subsection{Optimization models in renewable energy gen- eration}

Numerous optimization and simulation models have been developed to examine carbon pollution mitigation strategies and to analyze environmentally related energy policy issues. Some studies have not been linked to specific locations, but instead examine more-general questions. Hashim et al. (2005) provided such a study at the macro level, formulating an MILP model to reduce carbon pollution from a variety of EGUs, and constructing a simulation model to observe how the cost of a participant changes based on gross domestic product and $\mathrm{CO}_{2}$ emissions. Omu et al. (2013) presented such a study at a more micro level, developing an MILP model for the design of a distributed energy system that satisfies the electricity and heating demands of particular buildings to analyze the economic and environmental impact of the designed 
system. Numerous other studies have examined these issues in the context of specific locations, with many authors considering application in the EU. Georgiou (2016) examined the Greek power sector in terms of planning for long term electricity demand and achieving the country's EU emission reduction targets. An MILP model was developed to analyze the long term electricity source portfolio based on the country's minimum total electricity generation cost. Bigerna et al. (2016) investigated the cost of increasing renewable energy sources in EU power production under the target of the 2030 Framework for Climate and Energy Policies (European Commission 2016). Gómez-Calvet et al. (2016) proposed a two-stage analysis to assess the evaluation of environmental performance in the framework of EU countries. Another study that considers EU renewable energy targets was conducted by Zografidou et al. (2016). These authors applied a binary goal programming model to design the Greek renewable power generation network, accounting for environmental, social and economic perspectives. An example of a location-specific study positioned outside of the EU is provided by Chen et al. (2013), who presented an optimization model to reduce GHG emissions by optimizing the microgrid system of Taiwan considering the efficiency of electricity and heat generation.

A large number of studies examining environmental issues related to energy production have focused specifically on biomass and biofuels. Kim et al. (2011) presented an MILP model to maximize overall profit from biomass in biofuel production. They considered optimizing the number and locations of processing facilities, as well as the amount of biomass and derived products transported between locations. Ekşioğlu et al. (2009) developed a mathematical model that accounts for the design of the biomass supply chain. These authors considered the decisions associated with de- 
termining the location, inventory, and transportation required for biorefineries. An optimization model was proposed by Bentsen et al. (2014) to conceptualize energy supply for reducing GHG emissions via biomass resources under European Union (EU) energy policies. Song et al. (2012) proposed an MILP model that incorporates the use of sustainable waste in a regional energy system to mitigate GHG emissions and reduce dependence on fossil fuels. Many additional optimization models (Rentizelas et al. 2009, Van Dyken et al. 2010, Zhu \& Yao 2011) have been developed to design and analyze the supply chain of biomass for biofuel or biopower generation.

Woody biomass, in particular, has been the focus of many studies applying operations research models to biofuel and biopower generation. In Mobini et al. (2011), the authors investigated the supply of woody biomass from forests to a potential power plant with a simulation model to evaluate carbon emission from logistics operations. Shabani \& Sowlati (2013) studied a forest biomass supply chain management problem that includes procurement, storage, production, and ash management. They developed a nonlinear mixed integer programming model and applied it to an actual biomass power plant in Canada in order to determine the amount of forest biomass to be purchased, stored, and consumed for monthly planning over a one-year time horizon. Their objective function was to maximize profit, which is obtained by extracting biomass procurement cost, ash handling cost, storage cost, and production cost from the revenue of selling electricity. Alam, Pulkki, Shahi \& Upadhyay (2012) proposed an optimization model to estimate the procurement cost of woody biomass from forest management units to a biofuel generating facility in Canada. Other authors utilizing optimization models to examine woody biomass include Frombo et al. (2009), Alam, Pulkki, Shahi et al. (2012) and Zhang et al. (2014). 
Finally, a more limited set of studies have focused their attention on co-firing woody biomass with coal. Liu et al. (2014) examined woody biomass in co-firing in Missouri power plants. These authors developed a linear model to investigate the economic feasibility and the environmental effects of co-firing for existing power plants in Missouri. Hu et al. (2011) studied different types of biomass feedstocks, switchgrass and Miscanthhus, in co-firing for electricity generation to investigate the impact of co-firing on the $\mathrm{CO}_{2}$ and sulfur dioxide emissions in Taiwan.

\subsection{Robust optimization applications in renewable energy generation}

Robust optimization and stochastic optimization modeling take data uncertanity into account in order to reduce the risk of making an infeasible decision. In stochastic optimization, the probability distribution of the data parameters must be known. However, in robust optimization it is not necessary to determine the exact probabilty distribution for the data, instead the historical data could be sufficient to determine the range of potantial deviation for data parameters. Robust optimization was first introduced by Soyster (1973). His robust modeling approach did not consider the level of robustness in the uncertain parameter. Should the inexact parameters all deviate to their worst-case value, the optimal solution is still feasible for all possible scenarios, which could be considered as over-robust. In many applications, it is unlikely that

every potential worst-case variability would occur simultaneously. Decision makers may not prefer to make decisions by considering the worst case scenario due to its cost or "the price of robustness". 
Ben-Tal \& Nemirovski (1999) proposed a robust counterpart of a linear model in which an ellipsoidal uncertain data set was identified. With this ellipsoidal set, it could be possible make a decision that is less conservative compared to Soyster's modeling. In this way, the price of robustness could be reduced. Ben-Tal \& Nemirovski's robust counterpart model is conic quadratic which is computationally tractable with current commercial solvers. However, it could face computational difficulties for largescale problems.

Bertsimas \& Sim (2004) formulated the robust counterpart of a linear model using only linear functions. They used a $\Gamma$ input parameter that manages a budget of robustness for uncertain coefficients in both the objective function and constraints. In other words, this value allows decision makers to adjust the budget of robustness. Their model has received significant attention due to its computational tractability. Bertsimas \& Sim (2003) extended their robust modeling approach to discrete optimization and network flow problems. In this thesis, we will utilize this modeling approach to deal with data uncertainty in the boiler installation cost, coal electricity generation cost, and power plant emission rate.

Over the last two decades, robust optimization has been applied to different research fields such as economics, decision making, and energy generation planning. There are an increasing number of studies that have used robust optimization techniques to address the inexact data parameters in energy generation systems. Those techniques have often been used in renewable energy generation to reduce risks in the decision making. Gong et al. (2016) utilized a robust optimization technique in chemical optimization of the biocoversion and process network in order to deal with uncertainty in biomass feedstock price and biofuel product demand. They developed 
a two stage robust optimization model, in which the budget of robustness was used to control the level of uncertainty. Wang et al. (2015) proposed a robust optimization model for an energy generation scheduling problem in a microgrid systems that encompasses renewable energy generation, combined and heat power (CHP) generation and local heating systems. They considered the net electricity demand, the heat demand and electricity price as uncertain parameters. Parisio et al. (2012) applied a robust optimization model to an energy hub scheduling problem that consists of energy carriers, converting, storing and distributing electricity in order to satisfy electricity and heating demand. Their robust mixed integer model took into account the production efficiency of the energy converters in the hub as uncertain parameters. Koo et al. (2011) presented a robust optimization model for sustainable energy planning. They aimed to reduce the risk of infeasible decision making with respect to uncertain fuel costs and emission requirement levels. Tay et al. (2013) employed a scenario-based robust optimization model that determined the optimal integrated biorefinery configuration by considering biomass supply and product demand as uncertain parameters. Jiang et al. (2012) used a two-stage robust optimization-based unit commitment model to hedge wind output uncertainty on system operations. In order to solve the large-scale robust optimization problem,a Bender's decomposition algorithm was used to reduce the computation time. A technical report released by Moret et al. (2014) investigated the impact of uncertainty on long-term energy planning decisions. They proposed a robust MILP model that optimizes the technology choice with respect to minimizing energy cost to supply heat and electricity to a single family household. Foo et al. (2013) developed a scenario based robust LP and MILP model to mitigate the carbon footprint of transportation from the 
palm oil biomass residues sources to CHP power plants. Shabani \& Sowlati (2016) presented a hybrid stochastic-robust optimization model of a forest biomass supply chain. They used robust optimization to address uncertainty in biomass quality and biomass availability. To the best our knowledge, there is no study in the literature that addressees the uncertain parameters in co-firing woody biomass in coal-burning power plants to generate electricity while satisfying emission reduction targets by using robust optimization techniques.

\subsection{Demand response models to account for price- demand relationship}

In the literature regarding electricity generation, demand response models have been typcially utilized to optimize electricity consumption by adjusting electricity price at peak times of electricity consumption. However in this study we will utilize a demand response model to capture changes in the woody biomass price in the case of increased demand. The demand-price relationship has been investigated in various application in different ways. Kanudia \& Shukla (1998) presented a stochastic optimization approach for energy-environment planning. They incorporated the price sensitive demand in a linear formulation. Calfa \& Grossmann (2015) proposed a mixed integer nonlinear two-stage stochastic programming model that considers uncertainty in the demand and supply of a chemical process network. They also incorporated both a price-response model and a demand-response model in price optimization for a company. In the price response model, the demand $(d)$ is a function of the $\operatorname{price}(p)$, denoted by $d(p)$ whereas in the demand response model, the price is a function of the 
demand, presented as $p(d)$.

There have been some works that investigated the woody biomass demand and price relation from different aspects. From a local perspective, Du \& Runge (2014) investigated demand and woody biomass price relationship by determining a demand and supply function in the state of WI. They claimed that if the demand of the biofuel sector exceed a certain level per year, the woody biomass price would be significantly impacted by that. From a global perspective, Lauri et al. (2014) estimated global woody biomass energy potential utilizing the Global Biosphere Management Model (GLOBIOM) under different scenarios of hypothetical energy wood prices. In other words, they aimed to determine to the potential woody biomass supply when energy woody prices vary within certain ranges. The authors claimed that potential woody biomass could meet up to $16 \%$ of global energy consumption, even as household woody consumption might reach $18 \%$ of global energy consumption. Based on their estimates, from a regional perspective, woody biomass could satisfy $15 \%$ of North American energy consumption. Our study will take the complementary approach, considering the impact of increased woody biomass consumption on woody biomass price.

More generally, price elasticity of demand has been widely taken into account in decision modeling . The majority of these studies have focused on demand functions. Huang et al. (2013) presented a comprehensive survey of demand functions regarding decision modeling. The demand models they investigated rely on price, rebate, leadtime, space, quality, and advertising. They observed that the majority of the demand function were modeled in the functional forms of linear, power/iso-elastic, multinominal logit, and multiplicative competitive interaction. Kaplan et al. (2011) developed 
a mixed integer nonlinear programming (MINLP) model including the price elasticity of demand in optimization of supply chain systems. The authors specifically formulated the relationship between product selling price and demand. They included this price response model to estimate the revenue in the objective function. They used the GAMS platform to solve the convex MINLP model for a small instance, uncovering the relationship between the price and demand for a product in a supply chain network. In this study we utilized an iso-elastic, constant elasticity demand function. Thus, in this study we will assume that woody biomass price changes in response to increased woody biomass demand utilized to satisfy state-level and EPA emission reduction targets. Therefore, according to the notation of Calfa \& Grossmann (2015), we use a demand response model, in which the price is a function of the demand, represented by $p(d)$. To the best of our knowledge, there is no study in the literature that considered a demand response model in the optimization of bipower generation. 


\section{Chapter 3}

\section{Identifying Optimal Multi-state collaborations}

\subsection{Model Formulation}

We developed an MILP model that minimizes the total cost of generating the required electricity in a state, subject to environmental constraints, over a multi-year time horizon. The model identifies the optimal amount of electricity to generate from woody biomass and coal at each power plant identified as a candidate for co-firing. Moreover, the model also identifies any facility improvements to a power plant's material handling system (MHS) and boiler system that are necessary to meet environmental targets through increased use of woody biomass in co-firing.

The sets and indices, data parameters, and decision variables used in the MILP model are described below. 


\section{Sets and indices}

- $\mathcal{P}$ : set of all existing power plants that are candidates for co-firing biomass, indexed by $p$

- $\mathcal{R}$ : we identify the potential supply of woody biomass from logging residues and wood byproducts within a set of radii extending from each power plant, in particular, [0 km, $30 \mathrm{~km}$ ], (30 km, $60 \mathrm{~km}$ ], (60km, $90 \mathrm{~km}$ ], under the assumption that the cost of procuring woody biomass for each power plant will be different for material sourced within each radius ring; $\mathcal{R}$ then is the set of all such radii considered, indexed by $r$

- $\mathcal{S}$ : set of all states considered, indexed by $s$

- $\mathcal{T}$ : set of all time periods considered, in units of one year, indexed by $t$

\section{Data parameters}

- $\alpha_{p s}= \begin{cases}1 \quad \text { if plant } p \text { is in state } s \\ 0 \quad \text { otherwise }\end{cases}$

- $\sigma_{p}^{t}$ : cost to upgrade MHS at power plant $p$ during time period $t$ in order to use biomass in co-firing (assumes the upgrade will be completed at the beginning of time period $t$ )

- $\gamma_{p}^{t}$ : cost to upgrade the boiler at power plant $p$ during time period $t$ in order to increase the use of biomass in co-firing (assumes the upgrade will be completed at the beginning of time period $t$ )

- $\rho_{p}^{t}$ : cost to generate one megawatt-hour (MWh) from coal at power plant $p$ during time period $t$, including coal procurement cost 
- $\lambda_{p}^{t}$ : cost to generate one MWh from woody biomass at power plant $p$ during time period $t$, excluding fuel procurement cost

- $\xi$ : conversion rate from tons of woody biomass into MWh

- $\chi_{s}^{t}$ : annual renewable energy (RE) target, stated as MWh generated from wood and wood derived fuel, to be met by state $s$ during time period $t$

- $\beta_{s}^{t}$ : projected production requirements, in MWh, for electricity generated from coal to be met by state $s$ during time period $t$

- $\theta_{p r}^{t}$ : maximum potential supply of woody biomass from logging residues and wood byproducts that can be procured within radius $r$ of power plant $p$ during time period $t$

- $\pi_{p r}^{t}$ : total procurement cost to obtain all $\theta_{p r}^{t}$ tons of woody biomass available within radius $r$ of power plant $p$ during time period $t$

- $\bar{\pi}_{p}^{t}$ : fixed procurement cost necessary to obtain the initial woody biomass for power plant $p$ during time period $t$

- $\delta_{p s}^{t}$ : $\mathrm{CO}_{2}$ emission rate, in pounds per MWh, generated from coal at power plant $p$ during time period $t$

- $\phi_{s}^{t}$ : maximum $\mathrm{CO}_{2}$ emission rate allowed in state $s$ during time period $t$

- $\psi_{p}$ : maximum capacity for MWh generated from power plant $p$ during time period $t$

- $\epsilon$ : lower bound on the percentage of energy generated from woody biomass when a power plant has upgraded its MHS but not its boiler 
- $\zeta$ : upper bound on the percentage of energy generated from woody biomass when a power plant has upgraded its MHS but not its boiler

- $\mu_{p}$ : lower bound on the percentage of energy generated from woody biomass when power plant $p$ has upgraded its boiler

- $\eta_{p}$ : upper bound on the percentage of energy generated from woody biomass when power plant $p$ has upgraded its boiler

\section{Decision variables}

- $x_{p}^{t}= \begin{cases}1 & \text { if power plant } p \text { upgrades its MHS at at time } t \\ 0 & \text { otherwise }\end{cases}$

- $w_{p}^{t}= \begin{cases}1 & \text { if power plant } p \text { upgrades its boiler at time } t \\ 0 & \text { otherwise }\end{cases}$

- $v_{p}^{t}$ : total amount of woody biomass utilized at power plant $p$ during time period $t$

- $y_{p}^{t}$ : MWh generated from woody biomass at power plant $p$ during time period $t$

- $z_{p}^{t}:$ MWh generated from coal at power plant $p$ during time period $t$

- $a_{p}^{t}$ : MWh generated from woody biomass at power plant $p$ during time period $t$ if MHS upgrades, but not boiler upgrades, have been performed no later than time $t$

- $b_{p}^{t}$ : MWh generated from woody biomass at power plant $p$ during time period $t$ if boiler upgrades have been performed no later than time $t$ 
- $c_{p}^{t}$ : slack variable used in the fuel blending constraint for MHS upgrades when MHS upgrades have not yet been performed at power plant $p$ during time period $t$

- $d_{p}^{t}$ : slack variable used in the fuel blending constraint for MHS upgrades when boiler upgrades have already been performed at power plant $p$ during time period $t$

- $e_{p}^{t}$ : slack variable used in the fuel blending constraint for boiler upgrades when MHS upgrades, but not boiler upgrades, have already been performed at power plant $p$ during time period $t$

- $f_{p}^{t}$ : slack variable used in the fuel blending constraint for boiler upgrades when MHS upgrades have not yet been performed at power plant $p$ during time period $t$

- $g_{p r}^{t}$ : continuous variable used in piecewise linear function to determine amount of woody biomass utilized at power plant $p$ from sources within radius $r$ during time period $t$

- $\bar{g}_{p}^{t}$ : continuous variable used in piecewise linear function to determine amount of woody biomass utilized at power plant $p$ during time period $t$

- $h_{p r}^{t}$ : binary variable used in piecewise linear function to determine amount of woody biomass utilized at power plant $p$ from sources within radius $r$ during time period $t$

The MILP objective function and constraints are as follows. 


\section{Objective function}

Term (3.1) is the objective function, which aims to minimize total cost. This total cost includes power plants' operating expenses associated with each fuel type, potential capital investment expenses associated with facility upgrades, and fuel procurement costs.

$$
\begin{aligned}
\min \sum_{t \in \mathcal{T}} \sum_{p \in \mathcal{P}} \sigma_{p}^{t} x_{p}^{t}+\sum_{t \in \mathcal{T}} \sum_{p \in \mathcal{P}} \gamma_{p}^{t} w_{p}^{t}+\bar{\pi}_{p}^{t} \bar{g}_{p}^{t}+ & \sum_{t \in \mathcal{T}} \sum_{p \in \mathcal{P}} \sum_{r \in \mathcal{R}} \pi_{p r}^{t} g_{p r}^{t}+ \\
& \sum_{t \in \mathcal{T}} \sum_{p \in \mathcal{P}} \lambda_{p}^{t} y_{p}^{t}+\sum_{t \in \mathcal{T}} \sum_{p \in \mathcal{P}} \rho_{p}^{t} z_{p}^{t}
\end{aligned}
$$

\section{Constraints}

Constraint (3.2) converts the total amount of biomass utilized at power plant $p$ during time period $t$ into the generated MWh

$$
v_{p}^{t}=\frac{y_{p}^{t}}{\xi}, \quad \forall t \in \mathcal{T}, \quad \forall p \in \mathcal{P}
$$

Constraint (3.3) ensures that the total projected production requirement for electricity generation from coal and woody biomass during time period $t$ in state $s$ is satisfied by the total amount of electricity generated from all power plants in that state.

$$
\sum_{p \in \mathcal{P}} \alpha_{p s}\left(y_{p}^{t}+z_{p}^{t}\right) \geq \beta_{s}^{t}+\chi_{s}^{t}, \quad \forall t \in \mathcal{T}, \quad \forall s \in \mathcal{S}
$$

Constraint (3.4) limits the amount of electricity that can be generated from coal and woody biomass at power plant $p$ to be less than that plant's maximum capacity during 
time period $t$.

$$
y_{p}^{t}+z_{p}^{t} \leq \psi_{p}, \quad \forall t \in \mathcal{T}, \quad \forall p \in \mathcal{P}
$$

Constraint (3.5) ensures that the MWh generated from renewable resources in state $s$ during time period $t$ meets or exceeds regulatory lower bounds.

$$
\sum_{p \in \mathcal{P}} \alpha_{p s} y_{p}^{t} \geq \chi_{s}^{t}, \quad \forall t \in \mathcal{T}, \quad \forall s \in \mathcal{S}
$$

The $\mathrm{CO}_{2}$ emission rate is calculated based on the amount of $\mathrm{CO}_{2}$ released from coal EGUs divided by the amount of electricity generated from coal and woody biomass (EPA 2014a). Constraint (3.6) ensure that $\mathrm{CO}_{2}$ emissions per MWh from state $s$ during time period $t$ do not exceed the upper regulatory bound.

$$
\sum_{p \in \mathcal{P}} \alpha_{p s}\left(\frac{\delta_{p}^{t} z_{p}^{t}}{z_{p}^{t}+y_{p}^{t}}\right) \leq \phi_{s}^{t}, \quad \forall t \in \mathcal{T}, \quad \forall s \in \mathcal{S}
$$

Constraints (3.7) and (3.8) allow a power plant to upgrade each of its MHS and boiler, respectively, at most once during the model's time horizon. Constraint(3.9) is a precedence constraint, enforcing that a power plant cannot upgrade its boiler unless 
it has first upgraded its MHS.

$$
\begin{aligned}
\sum_{t \in \mathcal{T}} x_{p}^{t} & \leq 1, \quad \forall p \in \mathcal{P} \\
\sum_{t \in \mathcal{T}} w_{p}^{t} & \leq 1, \quad \forall p \in \mathcal{P} \\
w_{p}^{t} & \leq \sum_{t^{\prime} \leq t} x_{p}^{t^{\prime}}, \quad \forall t \in \mathcal{T}, \quad \forall p \in \mathcal{P}
\end{aligned}
$$

Constraints (3.10) through (3.20) are used to ensure that the amount of woody biomass-generated electricity at power plant $p$ during time period $t$ satisfies fuel blending constraints imposed by the facility upgrades that have been performed at the power plant by time period $t$. Constraints (3.10) through (3.13) define this amount of woody biomass-generated electricity as either $a_{p}^{t}$ (if only the MHS upgrade has been performed) or $b_{p}^{t}$ (if both upgrades have been performed).

$$
\begin{aligned}
& y_{p}^{t}=a_{p}^{t}+b_{p}^{t}, \quad \forall t \in \mathcal{T}, \quad \forall p \in \mathcal{P} \\
& a_{p}^{t} \leq M\left(\sum_{t^{\prime} \leq t} x_{p}^{t^{t^{\prime}}}\right), \quad \forall t \in \mathcal{T}, \quad \forall p \in \mathcal{P} \\
& a_{p}^{t} \leq M\left(1-\sum_{t^{\prime} \leq t} w_{p}^{t^{\prime}}\right), \quad \forall t \in \mathcal{T}, \quad \forall p \in \mathcal{P} \\
& b_{p}^{t} \leq M\left(\sum_{t^{\prime} \leq t} w_{p}^{t^{\prime}}\right), \quad \forall t \in \mathcal{T}, \quad \forall p \in \mathcal{P}
\end{aligned}
$$

Constraints (3.14) through (3.18) compute the values of slack variables needed for 
the fuel blending constraints.

$$
\begin{aligned}
& c_{p}^{t} \leq M\left(1-\sum_{t^{\prime} \leq t} x_{p}^{t^{\prime}}\right), \quad \forall t \in \mathcal{T}, \quad \forall p \in \mathcal{P} \\
& d_{p}^{t} \leq M\left(\sum_{t^{\prime} \leq t} w_{p}^{t^{\prime}}\right), \quad \forall t \in \mathcal{T}, \quad \forall p \in \mathcal{P} \\
& e_{p}^{t} \leq M\left(\sum_{t^{\prime} \leq t} x_{p}^{t^{\prime}}\right), \quad \forall t \in \mathcal{T}, \quad \forall p \in \mathcal{P} \\
& e_{p}^{t} \leq M\left(1-\sum_{t^{\prime} \leq t} w_{p}^{t^{\prime}}\right), \quad \forall t \in \mathcal{T}, \quad \forall p \in \mathcal{P} \\
& f_{p}^{t} \leq M\left(1-\sum_{t^{\prime} \leq t} x_{p}^{t^{\prime}}\right), \quad \forall t \in \mathcal{T}, \quad \forall p \in \mathcal{P}
\end{aligned}
$$

Constraint (3.19) ensures that if the MHS upgrade, but not the boiler upgrade, has been performed at power plant $p$ no later than time period $t$, then the percent of biomass generated-electricity at power plant $p$ during time period $t$ lies between the appropriate lower and upper bounds. Constraint (3.20) performs a similar role in the event that both the MHS and boiler upgrades have been performed at power plant $p$ no later than time period $t$.

$$
\begin{gathered}
\epsilon \leq \frac{a_{p}^{t}+c_{p}^{t}+d_{p}^{t}}{a_{p}^{t}+c_{p}^{t}+d_{p}^{t}+z_{p}^{t}} \leq \zeta, \quad \forall t \in \mathcal{T}, \quad \forall p \in \mathcal{P} \\
\mu_{s} \leq \frac{b_{p}^{t}+e_{p}^{t}+f_{p}^{t}}{b_{p}^{t}+e_{p}^{t}+f_{p}^{t}+z_{p}^{t}} \leq \eta_{s}, \quad \forall t \in \mathcal{T}, \quad \forall p \in \mathcal{P}
\end{gathered}
$$

We assume that the procurement cost per ton of woody biomass increases as the transport distance between the power plant and biomass source increases. Our model 
utilizes a piecewise-linear function to relate $v_{p}^{t}$, the amount of woody biomass utilized, to the cost of biomass procurement through the use of constraints (3.21) through $(3.26)$.

$$
\begin{gathered}
v_{p}^{t}=\sum_{r \in \mathcal{R}} \theta_{p r}^{t} g_{p r}^{t}, \quad \forall t \in \mathcal{T}, \quad \forall p \in \mathcal{P} \\
\bar{g}_{p}^{t}+\sum_{r \in \mathcal{R}} g_{p r}^{t}=1, \quad \forall t \in \mathcal{T}, \quad \forall p \in \mathcal{P} \\
\sum_{r \in \mathcal{R}} h_{p r}^{t}=1, \quad \forall t \in \mathcal{T}, \quad \forall p \in \mathcal{P} \\
\bar{g}_{p}^{t} \leq h_{p 1}^{t}, \quad \forall t \in \mathcal{T}, \quad \forall p \in \mathcal{P} \\
g_{p r}^{t} \leq h_{p r}^{t}+h_{p(r+1)}^{t}, \quad r=1 \ldots(|\mathcal{R}|-1), \quad \forall t \in \mathcal{T}, \quad \forall p \in \mathcal{P} \\
g_{p|\mathcal{R}|}^{t} \leq h_{p|\mathcal{R}|}^{t}, \quad \forall t \in \mathcal{T}, \quad \forall p \in \mathcal{P}
\end{gathered}
$$

The final constraints (3.27) and (3.28) impose binary and non-negativity constraints, respectively.

$$
\begin{array}{r}
x_{p}^{t}, w_{p}^{t}, h_{p r}^{t} \in\{0,1\}, \quad \forall t \in \mathcal{T}, \quad \forall t \in \mathcal{P} \\
v_{p}^{t}, y_{p}^{t}, z_{p}^{t}, a_{p}^{t}, b_{p}^{t}, c_{p}^{t}, d_{p}^{t}, e_{p}^{t}, f_{p}^{t}, g_{p r}^{t}, \bar{g}_{p}^{t} \geq 0, \quad \forall t \in \mathcal{T}, \quad \forall t \in \mathcal{P}
\end{array}
$$

\subsection{Data Integration}

\subsubsection{Power plants}

Information regarding candidate power plants for co-firing was obtained from eGrid data (EPA 2014b). Power plants were included for consideration in our analysis based 
on plant characteristics such as the use of stoker, cyclone and fluidized bed boilers on their premises which can feasibly incorporate the use of woody biomass without major facility upgrades (Aguilar et al. 2012). The set of candidate power plants for co-firing considering in this analysis included 14 plants in Missouri, 13 plants in Minnesota, 23 plants in Iowa, 20 plants in Wisconsin, and 21 plants in Illinois. Figure 3.1 presents a map showing these power plants' locations.

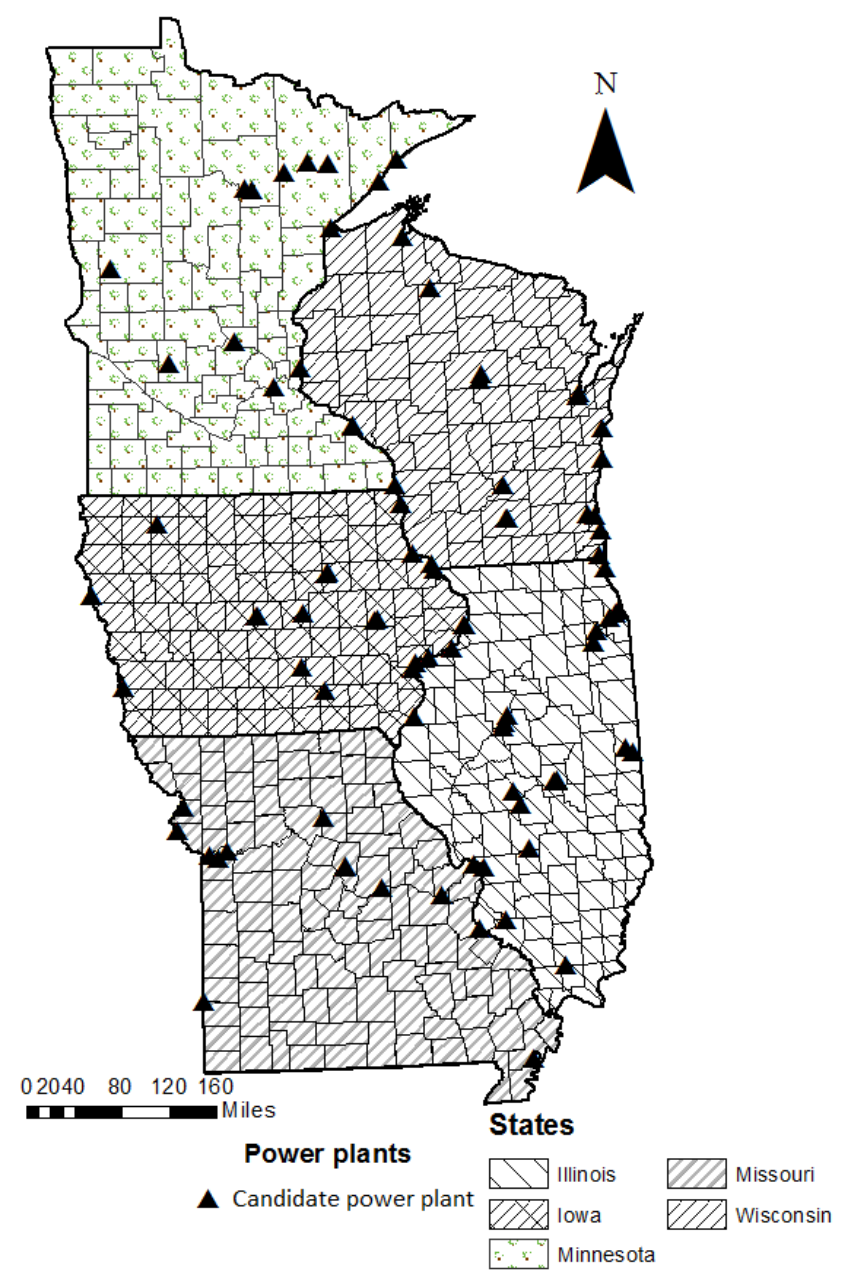

Figure 3.1: Locations of candidate power plants 


\subsubsection{Investment costs}

Power plant upgrades to the MHS are necessary to use woody biomass in co-firing. Based on information provided by the University of Missouri (MU) power plant, which recently upgraded its coal-fired cogeneration plant to co-fire woody biomass, we assumed, with only an MHS upgrade, a power plant will generate between 5\% and $10 \%$ of its total annual MWh from woody biomass. Based on the MU power plant's experience, we estimated a baseline MHS upgrading cost of $\$ 15$ million for a plant with a woody biomass nameplate generation capacity of $45 \mathrm{MWh}$. We define a plant's biomass nameplate generation capacity to be equal to the smaller of $200 \mathrm{MWh}$ or $50 \%$ of the plant's current nameplate capacity. MHS upgrade costs for other power plants were assumed to vary linearly from this baseline MHS upgrading cost with respect to each plant's biomass nameplate generation capacity. Power plants with historical biomass generation greater than $5 \%$ (as presented in the eGrid data (EPA 2014b)) were assumed to have the MHS upgrade already in place, with no upgrade cost required.

A power plant that has performed the MHS upgrade could elect to upgrade its boiler, allowing for increased use of biomass. We assume that, with a boiler upgrade, a power plant could generate between $5 \%$ of its coal-fired nameplate capacity plus $50 \%$ of its biomass nameplate capacity (as a lower bound) and $10 \%$ of its coal-fired nameplate capacity plus 100\% of its biomass nameplate capacity (as an upper bound). Based on communications with the engineering firm who assisted the MU power plant during its upgrade, we estimated a baseline boiler upgrading cost of $\$ 50$ million for a boiler with a nameplate capacity of 40MWh. Boiler upgrade costs for other power plants were assumed to vary linearly from this baseline boiler upgrading cost with 
respect to each plant's biomass nameplate generation capacity. Power plants with historical biomass generation greater than this lower bound (as presented in the eGrid data (EPA 2014b)) were assumed to have both the MHS and boiler upgrades already in place, with no upgrade cost required.

For either facility upgrade, we assume that the upgrade costs would be linearly depreciated over a 20-year lifetime. The costs included in this model would be the annual depreciation value over the interval from the time at which the upgrade occurs until 2030 (the end of our model's time horizon).

\subsubsection{Biomass conversion rate}

The amount of electricity that could be generated from one ton of woody biomass for each power plant is calculated based on the conversion rate published by Aguilar (2014) of $12,793 \mathrm{KJ} / \mathrm{Kg}$, which is equivalent to $3.55 \mathrm{MWh} /$ ton. However, some of this potential heat is lost in the co-firing process, depending on the moisture rate and size of the wood chips. Daigneault et al. (2012) estimated that $30 \%$ of this potential energy is typically captured in co-firing. Thus, this analysis assumes that the amount of electricity generated from woody biomass in co-firing is $1.07 \mathrm{MWh} /$ ton.

\subsubsection{Coal procurement cost and conversion rate}

The coal electricity generation cost per MWh encompasses a power plant's coal procurement cost per ton and its operation and maintenance $(O \& M)$ cost per MWh. The average price of coal per ton delivered to power plants was determined by considering the Annual Coal Report (EIA 2013), which provides a state-level coal cost per short 
ton delivered to the electric power sector. The US Energy Information Administration (EIA) (EIA 2016a) provides a conversion rate for coal of $1.904 \mathrm{MWh} /$ ton.

\subsubsection{O\&M costs}

The data associated with coal $O \& M$ and biomass $O \& M$ costs was obtained from a National Renewable Energy Laboratory (NREL) report (Tidball et al. 2010). Coal $O \& M$ is estimated as $\$ 27.52 / \mathrm{kW} / \mathrm{yr}$, which is equivalent $\$ 3.14$ per $\mathrm{MWh}$, whereas biomass $O \& M$ cost is $\$ 64.49 / \mathrm{kW} / \mathrm{yr}$, which is equivalent to $\$ 7.36$ per MWh. It is assumed that $O \& M$ costs are homogeneous across the candidate power plants considered in this research and do not change over the time horizon considered.

\subsubsection{Woody biomass availability}

We utilized data from Goerndt et al. (2012) to estimate total woody biomass availability within varying procurement radii of power plants. In their study, the USDA Forest Service Forest Inventory database was used to determine the total woody biomass available from logging residues (slash, by-products) and small diameter trees within a $30 \mathrm{~km}, 60 \mathrm{~km}$, and $90 \mathrm{~km}$ radius of power plants in the Northern US. This available biomass was computed at the county level, and was net of woody biomass growth, removals and mortality, and set not to increase annual biomass growth, such that biomass used for energy is not detrimental to existing standing biomass levels (Goerndt et al. 2012). We assumed that the total county-level woody biomass was uniformly distributed across that county. Based on the percentage of a county's land that falls within each radius of each power plant, the woody biomass potential for 
each power plant was estimated. It was further assumed that the amount of woody biomass that could be harvested at a sustainable rate would remain constant over the period 2022-2030.

\subsubsection{Woody biomass procurement cost}

The procurement cost per ton of woody biomass consists of the cost for stumpage, harvesting, chipping and transportation. This study utilizes the average procurement cost for woody biomass within a $30 \mathrm{~km}, 60 \mathrm{~km}$, and $90 \mathrm{~km}$ radius of each power plant, as estimated Goerndt et al. (2013).

\subsubsection{Electricity production requirements}

We utilized an EIA database (EIA 2014a) and states' RPS provided in the EPA proposal (EPA 2014a) in order to estimate the total electricity requirements in each state from coal and woody biomass in each year 2022-2030. To the best of our knowledge, future electricity production requirements at the state level have not been

estimated, these projections exist only at the regional level. We estimated future production requirements for coal-generated electricity at the state level using equation (3.29), where $T D^{t}$ is the total US electricity demand projection for time period $t, F C^{t}$ represents the projected percentage of US electricity generated by coal during time period $t$ (AEO 2013), $S C_{s}$ is the historical percentage of US coal-generated electricity produced in state $s$, and $I P_{s}$ is the percentage of the total coal-generated electricity nameplate capacity in state $s$ contained in the subset of power plants included in our 
analysis.

$$
\beta_{s}^{t}=T D^{t} * F C^{t} * S C_{s} * I P_{s}
$$

The estimated production requirements for woody-biomass generated electricity were computed in a similar manner; as presented in equation (3.30), where $F R^{t}$ is the projected percentage of US electricity generated by renewable resources other than hydropower during time period $t$ (AEO 2013), $S R_{s}$ is the historical percentage of US electricity generated by renewable resources other than hydropower produced in state $s, P B_{s}$ denotes the historical percentage of woody-biomass generated electricity relative to the total electricity generated in state $s$ by renewable resources other than hydropower, and $I B_{s}^{t}$ is the target percentage increase identified by EPA for future renewable electricity production in state $s$ during time period $t$.

$$
\chi_{s}^{t}=T D^{t} * F R^{t} * S R_{s} * P B_{s} * I B_{s}^{t}
$$

\subsubsection{Power plant capacity}

In the model, annual electricity generation capacity at each power plant is determined based upon eGrid data (EPA 2014b). As presented in equation (3.31), this is computed as the product of NameCap (the plant's nameplate capacity) and CapFac (the plant's historical utilization percentage), multiplied by 365 days/year times 24 hours/day to determine the maximum available MWh in any year. It is assumed that 
this value remains constant over all time periods $t$.

$$
\psi_{p}=\text { NameCap }_{p} * C a p F a c_{p} * 365 * 24
$$

\subsection{Marginal Cost Integrated Approach (MCIA)}

Initial attempts to solve the MILP presented in Section 3.1 while simultaneously considering all five states found the model to be impractical, due to an extremely long computational run time. For a single model run constrained to achieve the minimum-possible emission rate across all five states, a computational run time of more than 24 hours was required on a computer with an Intel Core i7-4710MQ (2.50 $\mathrm{GHz}$ ) processor and 8 GB RAM, with the MILP implemented using Python (version 3.2.5) (Python Software Foundation 2016) and solved by the Gurobi solver (Gurobi Optimization 2016).

Therefore, the modeling approach was modified to reduce the computational run time. A schematic representation of the marginal cost integrated approach (MCIA) that was utilized is presented in Figure 3.2. First, the MILP presented in Section 3.1 was solved for each individual state, across a range of required electricity generation levels and allowable $\mathrm{CO}_{2}$ emission levels. The required electricity levels ranged from the required renewable energy production in 2022 plus one million MWh, to the maximum possible production across all power plants considered in each state $s$, using increments of one million MWh. The allowable $\mathrm{CO}_{2}$ emission levels ranged from the minimum-achievable in each state $s$ at a total generation of one million $\mathrm{MWh}$, to the emission level generated if all power plants considered in state $s$ generated 


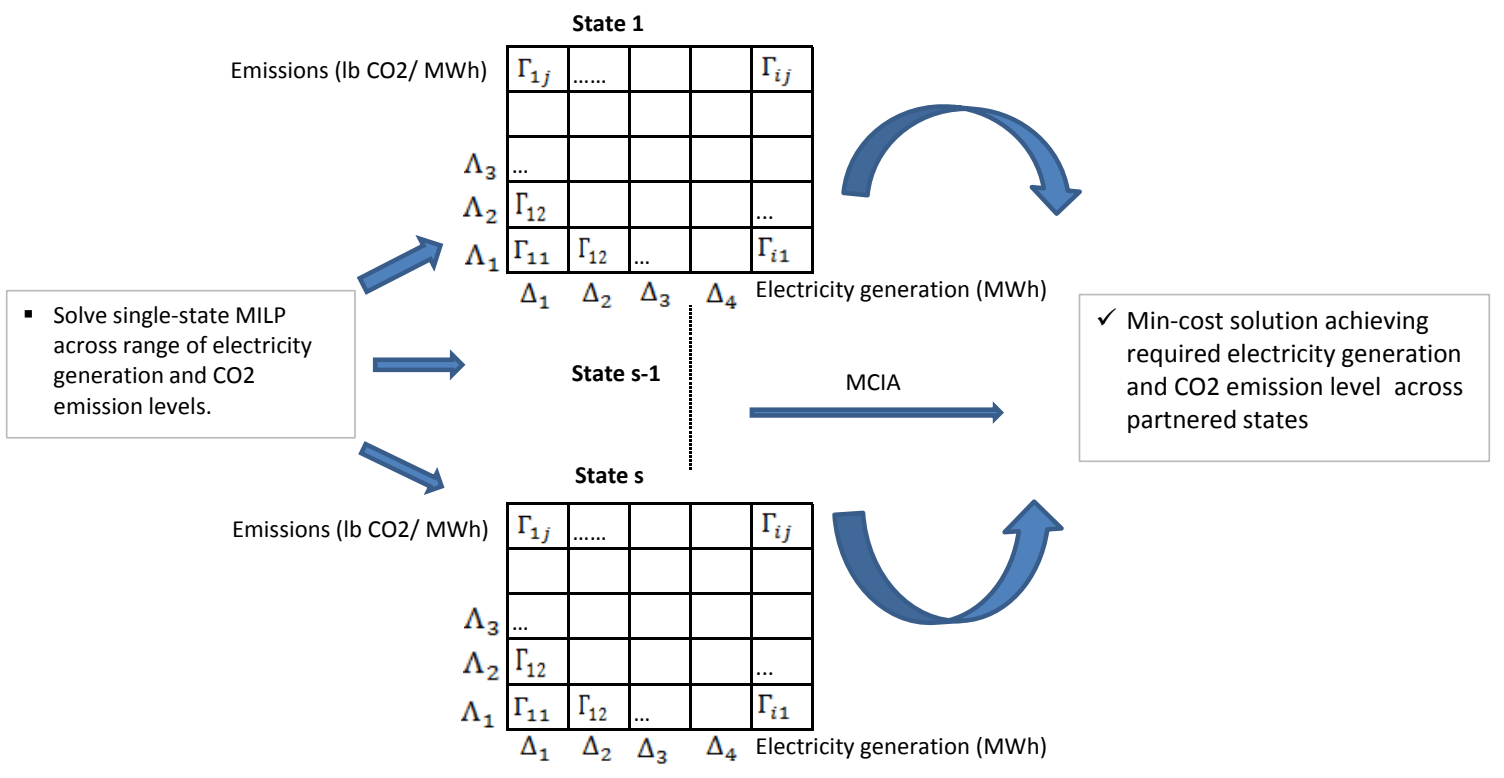

Figure 3.2: MCIA modeling approach

their maximum-possible amount of electricity from coal, with 10 total increments utilized between these upper and lower bounds. A separate MILP optimization run is required for each combination of required electricity level (substituting this value for the right-hand side term of constraint (3.3) for every time period $t$ ) and allowable $\mathrm{CO}_{2}$ emission level (substituting this value for the right-hand side term of constraint (3.6) for every time period $t$ ). The optimal cost identified by each of these model runs is then recorded in a marginal cost table (MCT), as shown in Figure 3.2. For each state, the MCT values present (depending upon one's orientation) either the marginal cost of generating an additional one million $\mathrm{MWh}$ at a constant $\mathrm{CO}_{2}$ emission rate, or the marginal cost of decreasing the $\mathrm{CO}_{2}$ emission rate by one increment at a constant MWh generation.

The MCIA model is represented using the following new sets and indices, data parameters, and decision variables: 


\section{Sets and indices}

- I: set of potential electricity generation levels, indexed by $i$

- $\mathcal{J}$ : set of potential $\mathrm{CO}_{2}$ emission levels, indexed by $j$

- $\overline{\mathcal{S}}$ : subset of states under consideration, indexed by $\bar{s} ; \overline{\mathcal{S}} \subseteq \mathcal{S}$

\section{Data parameters}

- $\Gamma_{i j \bar{s}}$ : cost to achieve electricity generation level $i$ at $\mathrm{CO}_{2}$ emission level $j$ in state $\bar{s}$ (in every year)

- $\Delta_{i \bar{s}}$ : MWh corresponding to electricity generation level $i$ in state $\bar{s}$

- $\Lambda_{j \bar{s}}: \mathrm{CO}_{2}$ emission rate, in pounds per MWh, corresponding to emission level $j$ in state $\bar{s}$

- $\Xi_{\bar{s}}$ : total MWh of required electricity generation in state $\bar{s}$ (based on year 2022)

- $\Omega$ : maximum allowable aggregate multi-state $\mathrm{CO}_{2}$ emission rate

\section{Decision variables}

$$
\text { - } u_{i j \bar{s}}= \begin{cases}1 \quad & \text { if electricity generation level } i \text { is achieved at } \mathrm{CO}_{2} \text { emission level } j \\ & \text { in state } \bar{s} \text { (in every year) } \\ 0 & \text { otherwise }\end{cases}
$$

The MCIA objective function and constraints are as follows.

\section{Objective function}

Term (3.32) is the objective function, which aims to minimize total cost.

$$
\min \sum_{i \in \mathcal{I}} \sum_{j \in \mathcal{J}} \sum_{\bar{s} \in \overline{\mathcal{S}}} \Gamma_{i j \bar{s}} u_{i j \bar{s}}
$$




\section{Constraints}

Constraint (3.33) ensures that total electricity generation satisfies the aggregate demand over all states under consideration.

$$
\sum_{i \in \mathcal{I}} \sum_{j \in \mathcal{J}} \sum_{\bar{s} \in \overline{\mathcal{S}}} \Delta_{i \bar{s}} u_{i j \bar{s}} \geq \sum_{\bar{s} \in \overline{\mathcal{S}}} \Xi_{\bar{s}}
$$

Constraint (3.34) ensures that aggregate $\mathrm{CO}_{2}$ emissions per MWh do not exceed the multi-state weighted emission target.

$$
\frac{\sum_{i \in \mathcal{I}} \sum_{j \in \mathcal{J}} \sum_{\bar{s} \in \overline{\mathcal{S}}} \Lambda_{j \bar{s}} \Delta_{i \bar{s}} u_{i j \bar{s}}}{\sum_{i \in \mathcal{I}} \sum_{j \in \mathcal{J}} \sum_{\bar{s} \in \overline{\mathcal{S}}} \Delta_{i \bar{s}} u_{i j \bar{s}}} \leq \Omega
$$

Constraint(3.35) ensures that exactly one electricity generation level and exactly one emission level is selected for each state under consideration.

$$
\sum_{i \in \mathcal{I}} \sum_{j \in \mathcal{J}} u_{i j \bar{s}}=1, \quad \forall \bar{s} \in \overline{\mathcal{S}}
$$

\subsubsection{Solving the MILP to generate input data for the MCIA}

It was first necessary to run the MILP presented in Section 3.1 multiple times for each individual state, populating the $\Gamma_{i j \bar{s}}$ values in the MCTs, in order to utilize the MCIA approach. Table 3.1 presents the computational run times, using the computer and software discussed earlier in this section, to generate the MCTs. Each scenario presented in the table requires a single MILP model run for one state with one required electricity level and one allowable $\mathrm{CO}_{2}$ emission level. Observe that for 
Table 3.1: Computational run time to generate MCTs

\begin{tabular}{|l|c|c|c|c|}
\hline State & $\begin{array}{c}\text { Number of } \\
\text { scenarios in MCT }\end{array}$ & $\begin{array}{c}\text { Total run } \\
\text { time (min) }\end{array}$ & $\begin{array}{c}\text { Average run time } \\
\text { per scenario (sec) }\end{array}$ & $\begin{array}{c}\text { Maximum run time } \\
\text { per scenario (sec) }\end{array}$ \\
\hline IL & 2,052 & 53.2 & 1.6 & 13.4 \\
\hline IA & 1,066 & 32.4 & 1.8 & 5.4 \\
\hline MN & 1,080 & 55.2 & 3.1 & 10.6 \\
\hline MO & 1,922 & 46.3 & 1.4 & 5.5 \\
\hline WI & 1,332 & 648.9 & 29.2 & 171.9 \\
\hline
\end{tabular}

every state except Wisconsin, each single-state MILP solved rather quickly: across the 6,210 total MILP model runs performed for IL, IA, MN and MO, the average run time was 1.8 seconds and the maximum run time was 13.4 seconds. However, the model ran much more slowly for WI; across its 1,332 scenarios the average run time was 29.2 seconds and the maximum run time was 171.9 seconds. The longer run times for WI are not obviously a function of problem size, as within our MILP, WI had more power plants under consideration than either MO or MN, but fewer than either IL or IA.

Having generated the MCTs, the MCIA can now be used to examine multi-state electricity generation strategies. The MCIA was coded using GAMS 24.4.6 and solved using CPLEX 12.6.2, again using the same computer.

\subsection{Results and Discussion}

In the remainder of this chapter, we will demonstrate how this modeling approach can be utilized to determine optimal partnering strategies for one state, namely, Missouri. The goal is to identify the multi-state partnerships that allow all partnering states to collectively generate their required electricity at a specified aggregate $\mathrm{CO}_{2}$ emission rate at minimum total cost. We assume here that Missouri has four potential partner 
states (Iowa, Illinois, Minnesota, Wisconsin), and that potential partnerships may be entered into with any subset of these states, including the empty set (in which case Missouri would operate alone), providing a total of 16 potential partnership combinations.

Recall that the initial MILP presented in Section 3.1 was unable to find an optimal solution within 24 hours of run time for a single model run that simultaneously considered all five states at one aggregate emission level. This model contained 24,582 variables, $34 \%$ of which were binary variables, and 28,890 constraints. A single run of the MCIA model presented in Section 3.3 that considered the same set of five states and same aggregate emission level was able to solve in 36.2 seconds; this MCIA had 15,581 variables, $42 \%$ of which were binary variables, and only 12 constraints. Of course, in order to utilize the MCIA, all five of the MCTs had to be populated (as discussed in Section 3.3.1), which required a total run time of 835.9 minutes. However, this is a one-time computational cost, and once it is completed the MCIA can then be used to examine any potential partnership at any potential emission level. Across the 480 instances considered $\left(30 \mathrm{CO}_{2}\right.$ emission levels for each potential partnership involving MO), the average MCIA run time was 76.4 seconds, demonstrating the computational efficiency of the MCIA approach.

Figure 3.3 presents the range of $\mathrm{CO}_{2}$ emission levels that can be achieved in each partnership, along with the associated minimum cost for each achievable $\mathrm{CO}_{2}$ emission level for each partnership, using increments of $10 \mathrm{lb} / \mathrm{MWh}$ to illustrate the shape of each partnership's tradeoff curve. For example, the situation in which Missouri operates alone corresponds to a curve that can achieve $\mathrm{CO}_{2}$ emission levels between 1,996 lb/MWh (at a cost of $\$ 24.13 / \mathrm{MWh}$ ) and 2,106 lb/MWh (at a cost of 


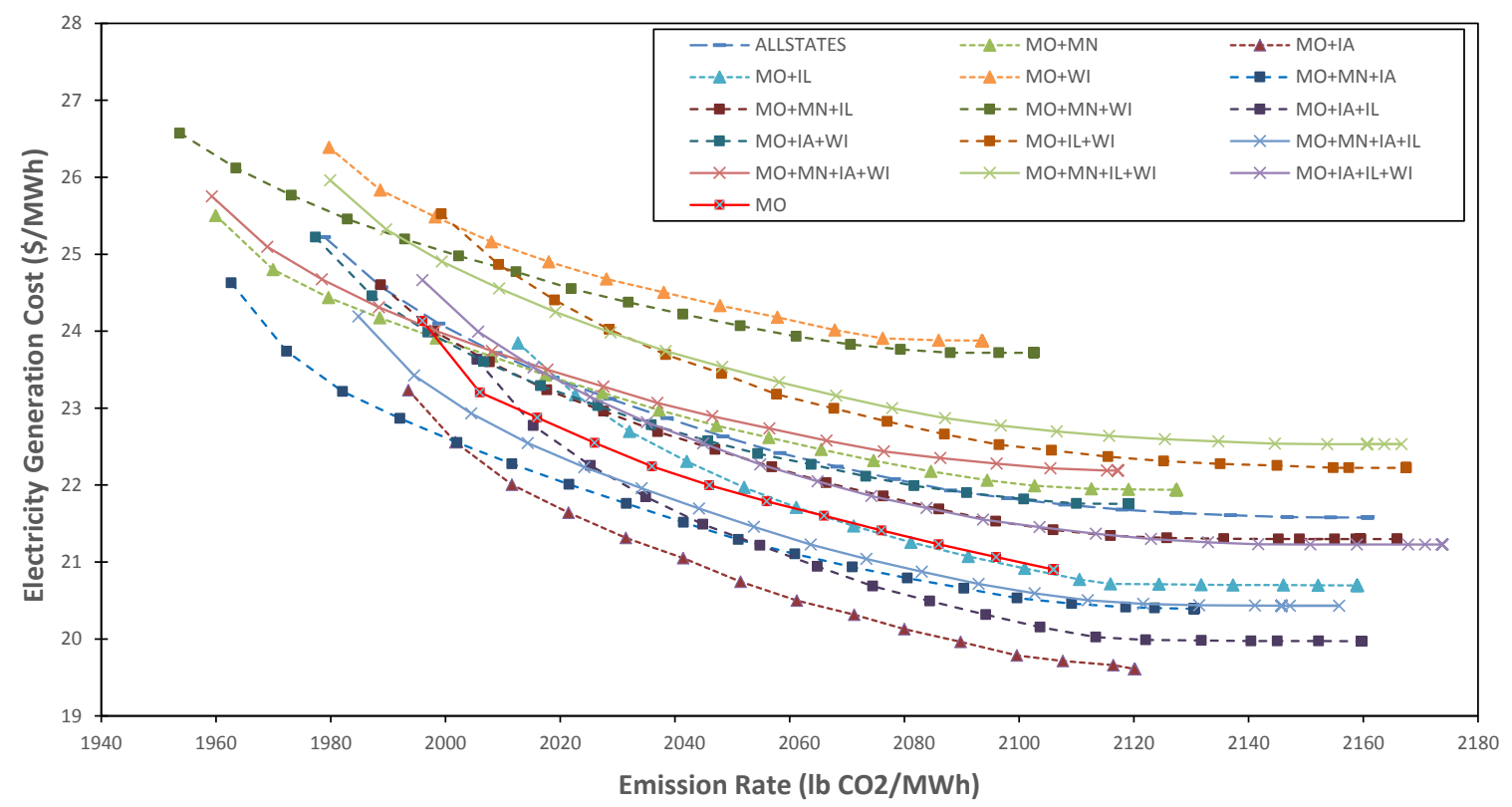

Figure 3.3: Cost versus emission rate tradeoff, all potential partnerships including Missouri

$\$ 20.90 / \mathrm{MWh}$ ). For the Missouri plants included in our analyses, the current emission rate is $2156 \mathrm{lb} / \mathrm{MWh}$. The graph in Figure 3.3 does not show a Missouri-only solution with emissions greater than $2106 \mathrm{lb} / \mathrm{MWh}$ because the optimal cost remains constant at $\$ 20.90$ /MWh for emission levels greater than $2106 \mathrm{lb} / \mathrm{Mwh}$ Alternatively, the situation in which Missouri partners with Minnesota corresponds to a curve that can achieve $\mathrm{CO}_{2}$ emission levels between 1,960 lb/MWh (at a cost of $\$ 25.50 / \mathrm{MWh}$ ) and 2,127 lb/MWh (at a cost of $\$ 21.94 / \mathrm{MWh}$ ). The primary reason that a lower aggregate $\mathrm{CO}_{2}$ emission rate can be achieved when Missouri partners with Minnesota is that Minnesota has a much larger amount of potential woody biomass located near candidate power plants (9.72 millions tons available annually within a $90 \mathrm{~km}$ radius, across all candidate power plants in Minnesota, versus 4.65 million tons in Missouri). 


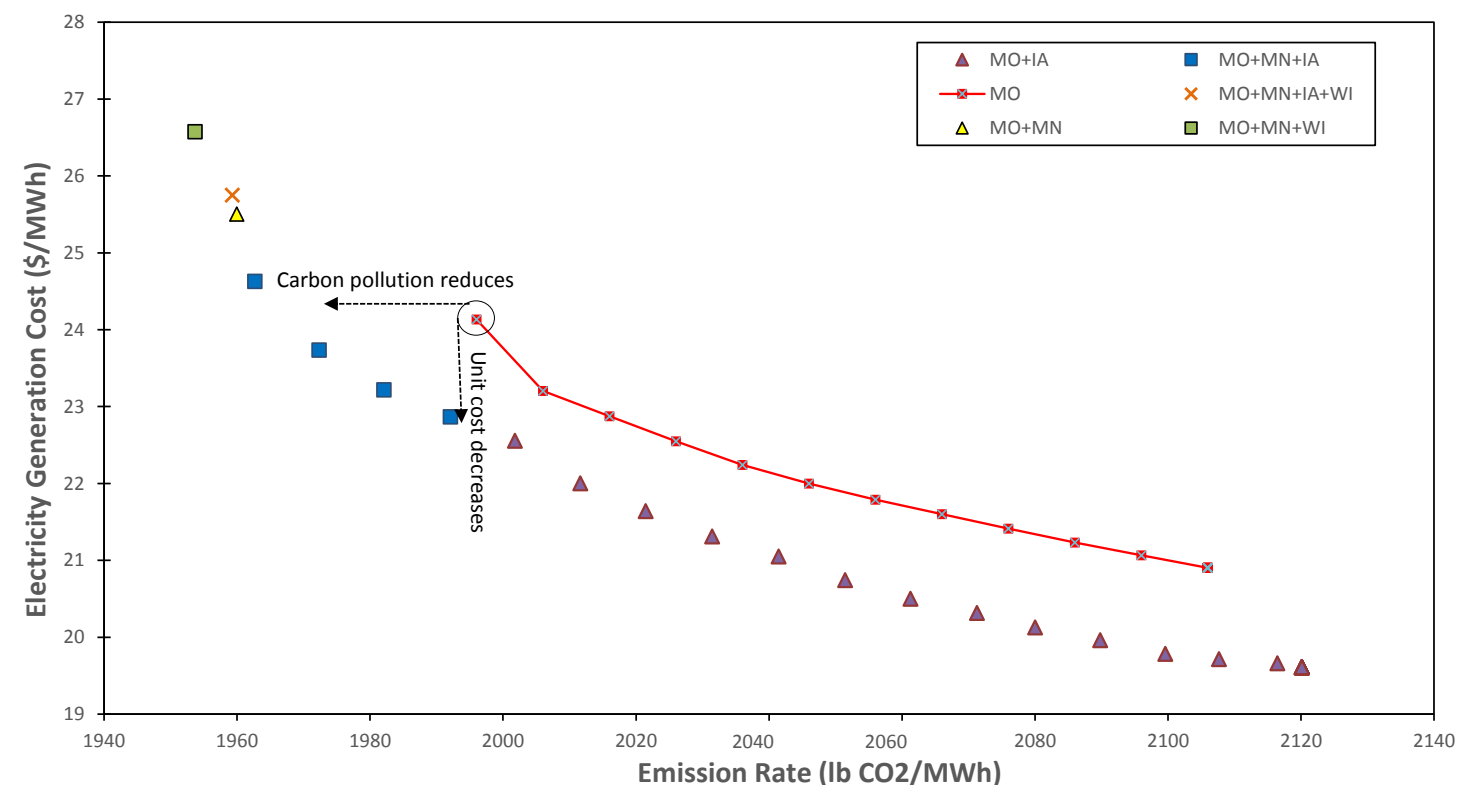

Figure 3.4: Efficient frontier of cost versus emission rate tradeoff, all potential partnerships including Missouri

Observe in Figure 3.3 that a given aggregate emission level (e.g., 2,020 lb/MWh) is achievable at a range of costs, depending upon the partnering arrangements selected (in this case, between $\$ 21.70 / \mathrm{MWh}$ if Missouri partners with Iowa and $\$ 24.86 / \mathrm{MWh}$ if MO partners with Wisconsin). Examining Figure 3.3, an efficient frontier can be identified across all potential partnerships, corresponding to all points for which a further reduction in the $\mathrm{CO}_{2}$ emission level is only possible at increased cost per MWh. This efficient frontier is presented in Figure 3.4; the tradeoff curve for the situation when Missouri operates independently is also presented for comparison.

Missouri's efficient frontier is comprised of five different partnerships:

- Missouri and Iowa; between $\mathrm{CO}_{2}$ emission rates of 2,120 lbs/MWh and 2,002 lbs/MWh

- Missouri, Minnesota and Iowa; between $\mathrm{CO}_{2}$ emission rates of 1,992 lbs/MWh 
and $1,963 \mathrm{lbs} / \mathrm{MWh}$

- Missouri and Minnesota; at $\mathrm{CO}_{2}$ emission rate of 1,960 lbs/MWh

- Missouri, Minnesota, Iowa and Wisconsin; at $\mathrm{CO}_{2}$ emission rate of 1,959 lbs per MWh

- Missouri, Minnesota and Wisconsin; at $\mathrm{CO}_{2}$ emission rate of 1,954 lbs/MWh

Observe that none of the solutions corresponding to Missouri operating alone lie on the efficient frontier, suggesting that multi-state partnerships are beneficial for Missouri at any $\mathrm{CO}_{2}$ emissions level. For example, the minimum $\mathrm{CO}_{2}$ emissions level that Missouri can achieve operating independently is 1,996 lbs/MWh, with an associated cost of $\$ 24.13 /$ MWh. If this emission level is viewed as acceptable, it could be achieved at approximately $6 \%$ less cost by partnering with Minnesota and Iowa. Alternatively, were this cost viewed as acceptable, it could be achieved with approximately $2 \%$ less $\mathrm{CO}_{2}$ emissions per MWh by again partnering with Minnesota and Iowa. Moreover, the minimum-achievable aggregate $\mathrm{CO}_{2}$ emission rate of 1,954 lb/MWh can only be achieved through partnering with Minnesota and Wisconsin, at a total cost of $\$ 26.48 / \mathrm{MWh}$. The minimum cost of $\$ 19.38 / \mathrm{MWh}$ can also only be achieved through partnering, in this case with Iowa, although this results in a $\mathrm{CO}_{2}$ emission level of 2,120 lbs/MWh.

As an illustration of the effects of multi-state collaborations, consider the minimumemissions level that can be acheived by Missouri when operating independently. As noted above, Missouri could achieve this aggregate emissions level of 1,996 lb $\mathrm{CO}_{2} / \mathrm{MWh}$ at minimum aggregate cost per MWh through a partnership with Minnesota and Iowa. Table 3.2 presents details on these solutions, along with the 
Table 3.2: Alternative Partnership Arrangements Generating Equal Emissions

\begin{tabular}{|c|c|c|c|c|c|}
\hline Partnership & State & $\begin{array}{l}\text { Ar } \\
\text { (milli } \\
\text { Coal }\end{array}$ & $\begin{array}{l}\text { generation } \\
\text { MWh) from: } \\
\text { Biomass }\end{array}$ & $\begin{array}{c}\text { Emission rate } \\
\left(\mathrm{lb} \mathrm{CO}_{2} / \mathrm{MWh}\right. \\
\text { biomass utilized }\end{array}$ & $\$ / \mathrm{MWh}$ \\
\hline Min-emissions for MO alone & MO & 48.0 & 3.0 & 1,996 & 24.13 \\
\hline Equal emissions for MN alone & $\mathrm{MN}$ & 22.4 & 2.1 & 1,996 & 25.18 \\
\hline Equal emissions for IA alone & IA & 32.0 & 1.5 & 1,996 & 21.09 \\
\hline Equal aggregate emissions & $\begin{array}{l}\mathrm{MO} \\
\mathrm{MN} \\
\mathrm{IA}\end{array}$ & $\begin{array}{l}49.9 \\
19.0 \\
33.2\end{array}$ & $\begin{array}{l}2.1 \\
3.8 \\
0.8\end{array}$ & $\begin{array}{l}2,026 \\
1,850 \\
2,040\end{array}$ & $\begin{array}{l}22.38 \\
28.52 \\
19.16\end{array}$ \\
\hline
\end{tabular}

minimum-cost solutions achieving 1,996 $\mathrm{lb} \mathrm{CO}_{2} / \mathrm{MWh}$ for Minnesota and Iowa if operating independently. The table shows the average annual MWh generated in each state by each fuel type (coal and biomass), the emission level achieved in each state, and the cost per MWh realized in each state.

Observe that Iowa can independently achieve an emissions rate of $1,996 \mathrm{lb} \mathrm{CO}_{2}$ per MWh at $13 \%$ less cost than can Missouri, while using relatively little biomass biomass providing $4.6 \%$ of the total MWh in Iowa versus $5.8 \%$ of the total MWh in Missouri. However, in order for Minnesota to independently achieve this emissions rate, it must generate $8.6 \%$ of its total MWh from biomass, and would experience a cost $4 \%$ greater than Missouri. Iowa can accrue this advantage because the set of its coal-burning power plants utilized in our model currently generates less emissions per MWh, on average, than do the coal-burning power plants from Missouri, which in turn have a lower level of emission rate than the average power plants in Minnesota.

Consider now the minimum-cost partnership between these three states that satisfies their aggregate electricity generation requirements at this aggregate emissions rate. Taking the per-state values presented in Table 3.2, weighted by each state's total MWh generation, we observe that this partnership achieves an aggregate emission rate of $1,993 \mathrm{lb} \mathrm{CO}_{2} / \mathrm{MWh}$ at an aggregate cost of $\$ 22.66 / \mathrm{MWh}$. The amount 
of electricity generated from biomass has increased very slightly in the partnership, to $6.2 \%$ in the partnership from a weighted average of $6.1 \%$ when each state operates independently. This outcome is the result of inter-state cooperation that yields an increase in electricity generated from biomass in MN that is slightly larger than the corresponding reductions in the other two states.

Note, however, that while the cost per MWh under the partnership has reduced by $7 \%$ and $9 \%$ in MO and IA, respectively, due to lower utilization of biomass for cofiring, the cost increased by $13 \%$ in $\mathrm{MN}$, due to its $82 \%$ increase in MWh generated from biomass. This outcome is consistent with the greater availability of biomass feedstocks at relatively shorter distances from the power plants in MN, allowing its power plants to exercise their relative advantage to utilize biomass to reduce the partnership's aggregate emissions.

Of course, some sort of arrangement would be needed whereby the beneficiaries in the partnership (in this case, MO and IA) compensate the payer (here, MN), such that the overall net cost reduction is shared across all partners. For this situation, a simple notional scheme consists of transfer payments by MO and IA to MN equal to $4.43 \%$ and $6.57 \%$, respectively, of the total generation cost in MO and IA. Under such an arrangement each state's cost per MWh (after the transfers) would be reduced by $3.1 \%$ relative to its independent minimum-achievable cost at an emissions rate of 1,996 lb $\mathrm{CO}_{2} / \mathrm{MWh}$. While considerable analysis would be needed to construct such transfer arrangements in an equitable manner so as to generate stable partnerships and minimize transaction costs, the realization that partnerships can achieve such significant cost reductions for all partners would arguably be a strong motivation for their creation. 
Table 3.3: Emissions and renewable energy percentage, target and current values, by state

\begin{tabular}{l|cc|cc}
\hline State & $\begin{array}{c}\text { EPA } \\
\text { target }\end{array}$ & $\begin{array}{c}\text { Emissions (lb CO } \mathbf{C O}_{2} / \mathbf{M W h} \text { ) } \\
\text { included in analysis }\end{array}$ & $\begin{array}{c}\text { RPS } \\
\text { target }\end{array}$ & $\begin{array}{c}\text { Renewable energy } \\
\text { included in analysis }\end{array}$ \\
\hline IL & 1,245 & 2,225 & $7 \%$ & $0.0002 \%$ \\
IA & 1,283 & 2,215 & $15 \%$ & $0.14 \%$ \\
MN & 1,213 & 2,230 & $15 \%$ & $1.09 \%$ \\
MO & 1,272 & 2,156 & $2 \%$ & $0.02 \%$ \\
WI & 1,176 & 2,011 & $8 \%$ & $0.80 \%$ \\
\hline
\end{tabular}

Consider now the EPA emission targets and state RPS targets that motivated this research; Table 3.3 presents these values for the five states under consideration, along with the current emission rate across all power plants considered in our analysis. For the five states considered, the $\mathrm{CO}_{2}$ emission rates that can be achieved through co-firing biomass in existing coal-fired power plants fall considerably short of the full EPA targets. However, recall that these emission targets are applied statewide, to total electricity generation from all sources. For Missouri, aggregate emission reductions of greater than $9 \%$ below current levels can be achieved at existing coal-fired power plants through co-firing biomass in partnership with Minnesota and Wisconsin, making a sizeable contribution toward this overall goal that could potentially be achieved relatively quickly and at moderate additional expense, while longer-term investments are made in other renewable EGUs.

RPS targets can be met using a combination of renewable resources, such as solar, wind, and biomass. Figure 3.5 presents the percentage of energy generated from renewable resources (i.e., woody biomass) for each of the solutions lying on Missouri's efficient frontier. Observe that the Missouri RPS target of $2 \%$ can be greatly exceeded for this set of power plants (which have previously relied almost 


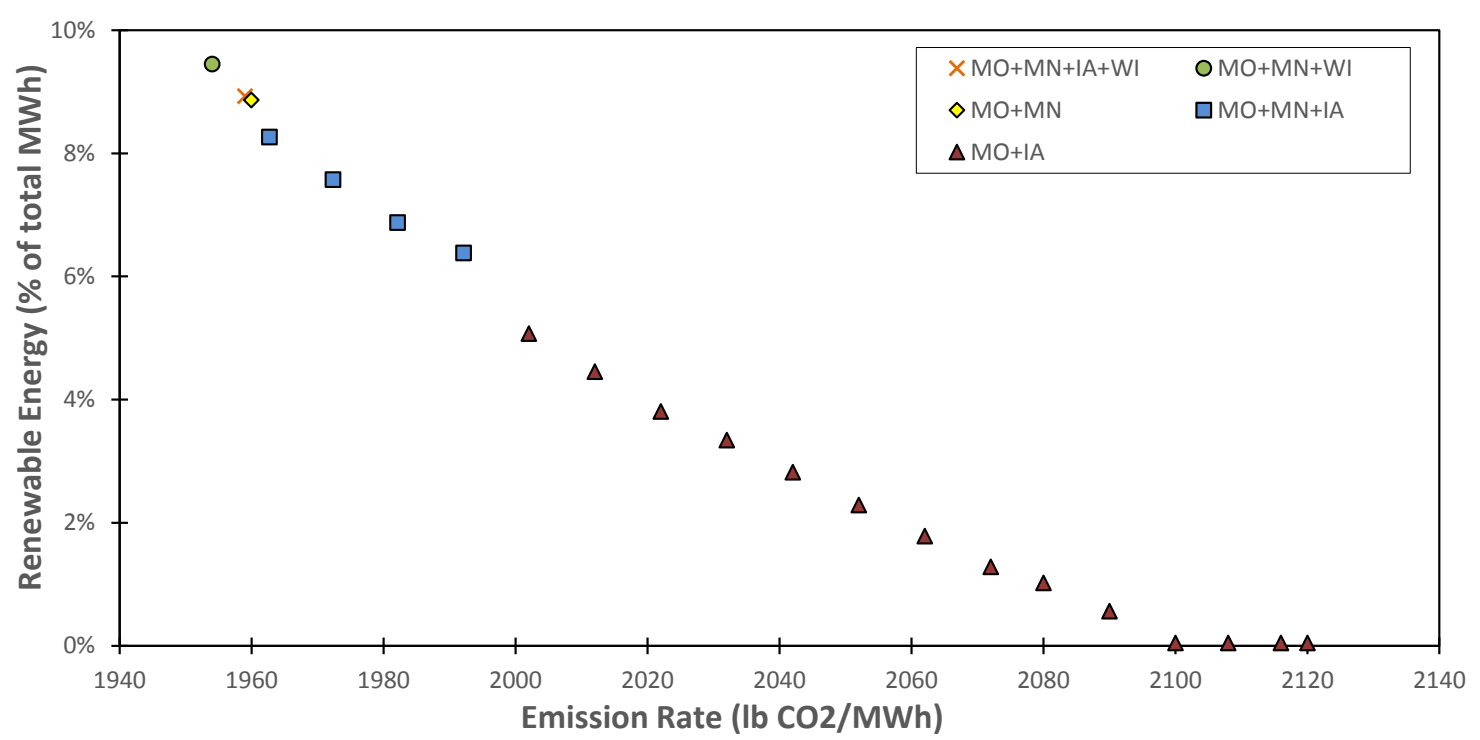

Figure 3.5: Renewable energy percentage versus emission rate tradeoff, for points on efficient frontier of cost versus emission rate for all potential partnerships including Missouri

exclusively on burning coal, historically generating $0.02 \%$ of their electricity from renewable resources). The minimum-emission strategy, in which Missouri is partnered with Minnesota and Wisconsin, can generate $9.5 \%$ of its electricity from renewable resources, exceeding the RPS requirements for Missouri and Wisconsin, but falling short of the $15 \%$ RPS target for Minnesota. 


\section{Chapter 4}

\section{Accounting for Uncertainty in Identifying Optimal Multi-state collaborations}

\subsection{Robust Counterpart Formulations}

This chapter presents an RO formulation that builds upon the MILP presented in section 3.1 to account for uncertainty in three data parameters: coal electricity generation cost, boiler installation cost, and power plant $\mathrm{CO}_{2}$ emission rate. This $\mathrm{RO}$ model minimizes the total cost of generating the required electricity in a state, subject to environmental constraints, over a multi-year time horizon, according to the user's pre-specified level of robustness. The model identifies the optimal amount of electricity to generate from woody biomass and coal at each power plant identified as a candidate for co-firing. The model also identifies any facility improvements to a power plant's material handling system (MHS) and boiler system that are necessary 
to meet environmental targets through increased use of woody biomass in co-firing.

We assume in this RO formulation that uncertainty exists in the objective function and in the maximum-allowable emissions constraint. The other constraints, which do not contain uncertain data parameters, remain unchanged from the MILP formulation of section 3.1. Accordingly, this section will discuss only the objective function and emissions constraint; the entire RO MILP formulation, including those constraints unchanged from section 3.1, is, however, presented in this chapter.

The sets and indices, data parameters, and decision variables used in the objective function and emissions constraint of the RO MILP model are described below.

\section{Sets and indices}

- $\mathcal{P}$ : set of all existing power plants that are candidates for co-firing biomass, indexed by $p$; with three subsets defined as follows

$$
\begin{aligned}
& -P_{s}^{t}=\left\{p \mid \hat{\delta}_{p}^{t}>0, \alpha_{p s}=1\right\} \\
& -\tilde{P}=\left\{(t, p) \mid \hat{\gamma}_{p}^{t}>0\right\} \\
& -\dot{P}=\left\{(t, p) \mid \hat{\rho}_{p}^{t}>0\right\}
\end{aligned}
$$

- $\mathcal{R}$ : we identify the potential supply of woody biomass from logging residues and wood byproducts within a set of radii extending from each power plant, in particular, [0 km, $30 \mathrm{~km}],(30 \mathrm{~km}, 60 \mathrm{~km}],(60 \mathrm{~km}, 90 \mathrm{~km}]$, under the assumption that the cost of procuring woody biomass for each power plant will be different for material sourced within each radius ring; $\mathcal{R}$ then is the set of all such radii considered, indexed by $r$

- $\mathcal{S}$ : set of all states considered, indexed by $s$

- $\mathcal{T}$ : set of all time periods considered, in units of one year, indexed by $t$ 


\section{Data parameters}

- $\alpha_{p s}= \begin{cases}1 & \text { if plant } p \text { is in state } s \\ 0 & \text { otherwise }\end{cases}$

- $\sigma_{p}^{t}$ : cost to upgrade MHS at power plant $p$ during time period $t$ in order to use biomass in co-firing (assumes the upgrade will be completed at the beginning of time period $t$ )

- $\bar{\gamma}_{p}^{t}$ : nominal cost to upgrade the boiler at power plant $p$ during time period $t$ in order to increase the use of biomass in co-firing (assumes the upgrade will be completed at the beginning of time period $t$ )

- $\hat{\gamma}_{p}^{t}$ : maximum-allowable deviation of the cost to upgrade the boiler at power plant $p$ during time period $t$ in order to increase the use of biomass in co-firing (assumes the upgrade will be completed at the beginning of time period $t$ )

- $\bar{\rho}_{p}^{t}$ : nominal cost to generate one megawatt-hour (MWh) from coal at power plant $p$ during time period $t$, including coal procurement cost

- $\hat{\rho}_{p}^{t}$ : maximum-allowable deviation of the cost to generate one megawatt-hour (MWh) from coal at power plant $p$ during time period $t$, including coal procurement cost

- $\Gamma_{0}$ : robustness level of the objective function

- $\lambda_{p}^{t}$ : cost to generate one MWh from woody biomass at power plant $p$ during time period $t$, excluding fuel procurement cost

- $\xi$ : conversion rate from tons of woody biomass into MWh

- $\chi_{s}^{t}$ : annual renewable energy (RE) target, stated as MWh generated from wood and wood derived fuel, to be met by state $s$ during time period $t$

- $\beta_{s}^{t}$ : projected production requirements, in MWh, for electricity generated from 
coal to be met by state $s$ during time period $t$

- $\theta_{p r}^{t}$ : maximum potential woody biomass supply from logging residues and wood byproducts that can be procured within radius $r$ of power plant $p$ during time period $t$

- $\pi_{p r}^{t}$ : total procurement cost to obtain all $\theta_{p r}^{t}$ tons of woody biomass available within radius $r$ of power plant $p$ during time period $t$

- $\bar{\pi}_{p}^{t}$ : fixed procurement cost necessary to obtain the initial woody biomass for power plant $p$ during time period $t$

- $\bar{\delta}_{p}^{t}$ : nominal $\mathrm{CO}_{2}$ emission rate, in pounds per $\mathrm{MWh}$, generated from coal at power plant $p$ during time period $t$

- $\hat{\delta}_{p}^{t}$ : maximum-allowable deviation of the $\mathrm{CO}_{2}$ emission rate, in pounds per MWh, generated from coal at power plant $p$ during time period $t$

- $\Gamma_{s}^{t}$ : robustness level of the emissions constraint

- $\phi_{s}^{t}$ : maximum $\mathrm{CO}_{2}$ emission rate allowed in state $s$ during time period $t$

- $\psi_{p}$ : maximum capacity for MWh generated from power plant $p$ during time period $t$

- $\epsilon$ : lower bound on the percentage of energy generated from woody biomass when a power plant has upgraded its MHS but not its boiler

- $\zeta$ : upper bound on the percentage of energy generated from woody biomass when a power plant has upgraded its MHS but not its boiler

- $\mu_{s}$ : lower bound on the percentage of energy generated from woody biomass when power plant $s$ has upgraded its boiler

- $\eta_{s}$ : upper bound on the percentage of energy generated from woody biomass when power plant $s$ has upgraded its boiler 


\section{Decision variables}

- $x_{p}^{t}= \begin{cases}1 \quad \text { if power plant } p \text { upgrades its MHS at at time } t \\ 0 \quad \text { otherwise }\end{cases}$

- $w_{p}^{t}= \begin{cases}1 & \text { if power plant } p \text { upgrades its boiler at time } t \\ 0 & \text { otherwise }\end{cases}$

- $v_{p}^{t}$ : total amount of woody biomass utilized at power plant $p$ during time period

$t$

- $y_{p}^{t}$ : MWh generated from woody biomass at power plant $p$ during time period $t$

- $z_{p}^{t}$ : MWh generated from coal at power plant $p$ during time period $t$

- $a_{p}^{t}$ : MWh generated from woody biomass at power plant $p$ during time period $t$ if MHS upgrades, but not boiler upgrades, have been performed no later than time $t$

- $b_{p}^{t}$ : MWh generated from woody biomass at power plant $p$ during time period $t$ if boiler upgrades have been performed no later than time $t$

- $c_{p}^{t}$ : slack variable used in the fuel blending constraint for MHS upgrades when MHS upgrades have not yet been performed at power plant $p$ during time period $t$

- $d_{p}^{t}$ : slack variable used in the fuel blending constraint for MHS upgrades when boiler upgrades have already been performed at power plant $p$ during time period $t$

- $e_{p}^{t}$ : slack variable used in the fuel blending constraint for boiler upgrades when MHS upgrades, but not boiler upgrades, have already been performed at power plant $p$ during time period $t$

- $f_{p}^{t}$ : slack variable used in the fuel blending constraint for boiler upgrades when 
MHS upgrades have not yet been performed at power plant $p$ during time period $t$

- $g_{p r}^{t}$ : continuous variable used in piecewise linear function to determine amount of woody biomass utilized at power plant $p$ from sources within radius $r$ during time period $t$

- $\bar{g}_{p}^{t}$ : continuous variable used in piecewise linear function to determine amount of woody biomass utilized at power plant $p$ during time period $t$

- $h_{p r}^{t}$ : binary variable used in piecewise linear function to determine amount of woody biomass utilized at power plant $p$ from sources within radius $r$ during time period $t$

- $\ell$ : robustness variable for the objective function

- $\tilde{m}_{p}^{t}$ : robustness variable for boiler upgrade cost in the objective function

- $\dot{m}_{p}^{t}$ : robustness variable for coal electricity generation cost in the objective function

- $\tilde{n}_{p}^{t}$ : robustness variable for boiler upgrade cost in the objective function

- $\dot{n}_{p}^{t}$ : robustness variable for coal electricity generation cost in the objective function

- $\breve{\ell}_{s}^{t}$ : robustness variable for the emissions constraint

- $\breve{m}_{p s}^{t}$ : robustness variable for the emissions constraint

- $\breve{n}_{p}^{t}$ : robustness variable for the emissions constraint

\subsubsection{Robust counterpart of the objective function}

Term (4.1) is the objective function, which aims to minimize total cost, subject to a user-specified level of robustness. This total cost includes power plants' operat- 
ing expenses associated with each fuel type, potential capital investment expenses associated with facility upgrades, and fuel procurement costs.

$$
\begin{array}{r}
\min \sum_{t \in \mathcal{T}} \sum_{p \in \mathcal{P}} \sigma_{p}^{t} x_{p}^{t}+\sum_{t \in \mathcal{T}} \sum_{p \in \mathcal{P}} \bar{\gamma}_{p}^{t} w_{p}^{t}+\bar{\pi}_{p}^{t} \bar{g}_{p}^{t}+\sum_{t \in \mathcal{T}} \sum_{p \in \mathcal{P}} \sum_{r \in \mathcal{R}} \pi_{p r}^{t} g_{p r}^{t}+\sum_{t \in \mathcal{T}} \sum_{p \in \mathcal{P}} \lambda_{p}^{t} y_{p}^{t}+ \\
\sum_{t \in \mathcal{T}} \sum_{p \in \mathcal{P}} \bar{\rho}_{p}^{t} z_{p}^{t}+\ell \Gamma_{0}+\sum_{(t, p) \in \tilde{\mathcal{P}}} \tilde{m}_{p}^{t}+\sum_{(t, p) \in \dot{\mathcal{P}}} \dot{m}_{p}^{t}
\end{array}
$$

The final three terms of this objective function, which account for robustness, are based on the RO formulation of Bertsimas \& Sim (2003) to account for input data uncertainty. The objective function coefficients that were assumed to be uncertain are the boiler installation cost and the coal electricity generation cost. The uncertain boiler installation cost is denoted $\gamma_{p}^{t}$; we assume that this parameter is bounded and varies symmetrically inside the interval $\left[\bar{\gamma}_{p}^{t}-\hat{\gamma}_{p}^{t}, \bar{\gamma}_{p}^{t}+\hat{\gamma}_{p}^{t}\right]$, where $\bar{\gamma}_{p}^{t}$ is the nominal value of the boiler upgrading cost and $\hat{\gamma}_{p}^{t}$ is the maximum-allowable deviation (halfwidth) of potential values. The coal electricity generation cost, $\rho_{p}^{t}$, is another uncertain parameter in the objective function; this parameter is assumed to take a value in $\left[\bar{\rho}_{p}^{t}-\hat{\rho}_{p}^{t}, \bar{\rho}_{p}^{t}+\hat{\rho}_{p}^{t}\right]$ and is similarly assumed to vary symmetrically over this interval, where $\bar{\rho}_{p}^{t}$ is the nominal value of coal electricity generation cost and $\hat{\rho}_{p}^{t}$ is its maximum-allowable deviation (halfwidth). It is further assumed that all uncertain parameters vary independently of one another.

Let $\tilde{P}=\left\{(t, p) \mid \hat{\gamma}_{p}^{t}>0\right\}$ and $\dot{P}=\left\{(t, p) \mid \hat{\rho}_{p}^{t}>0\right\}$; that is, $\tilde{P}$ and $\dot{P}$ are the respective subsets of the boiler upgrading cost and coal electricity generation cost parameters that can potentially deviate from their nominal values. Bertsimas \& Sim (2004) proposed input parameter $\Gamma_{0}$, which must take an integer value, to allow the user to control the level of robustness in the objective function. When the user sets 
$\Gamma_{0}=0$, it means that no parameters are allowed to deviate from their nominal values; what remains is a non-robust model. In our study's model, when $\Gamma_{0}=|\tilde{P}|+|\dot{P}|$, all parameters in subsets $\tilde{P}$ and $\dot{P}$ are allowed to deviate from their nominal values; in this case the optimal solutions that are obtained are based on the worst-case scenario, as in Soyster (1973). For an intermediate value of $\Gamma_{0}$ between these two endpoints, the terms $\ell \Gamma_{0}+\sum_{(t, p) \in \tilde{\mathcal{P}}} \tilde{m}_{p}^{t}+\sum_{(t, p) \in \mathcal{P}} \dot{m}_{p}^{t}$ in objective (4.1) compute the additional cost (above the nominal levels) associated with the $\Gamma_{0}$ worst-case-impact values of decision variables $w_{p}^{t}$ and $z_{p}^{t}$.

To control for the desired level of robustness $\Gamma_{0}$ in objective function (4.1), it is necessary to add constraints (4.2) - (4.9), which are presented in below.

$$
\begin{array}{rc}
\ell+\tilde{m}_{p}^{t} \geq \hat{\gamma}_{p}^{t} \tilde{n}_{p}^{t} & \forall(t, p) \in \tilde{\mathcal{P}} \\
\ell+\dot{m}_{p}^{t} \geq \hat{\rho}_{p}^{t} \dot{n}_{p}^{t} & \forall(t, p) \in \dot{\mathcal{P}} \\
\tilde{m}_{p}^{t} \geq 0 & \forall(t, p) \in \tilde{\mathcal{P}} \\
\dot{m}_{p}^{t} \geq 0 & \forall(t, p) \in \dot{\mathcal{P}} \\
\tilde{n}_{p}^{t}, \dot{n}_{p}^{t} \geq 0 & \forall t \in \mathcal{T}, \quad \forall p \in \mathcal{P} \\
\ell \geq 0 & \\
-\tilde{n}_{p}^{t} \leq w_{p}^{t} \leq \tilde{n}_{p}^{t} & \forall t \in \mathcal{T}, \quad \forall p \in \mathcal{P} \\
-\dot{n}_{p}^{t} \leq z_{p}^{t} \leq \dot{n}_{p}^{t} & \forall t \in \mathcal{T}, \quad \forall p \in \mathcal{P}
\end{array}
$$




\subsubsection{The uncertainty in the constraint data parameters}

The $\mathrm{CO}_{2}$ emission rate is calculated based on the amount of $\mathrm{CO}_{2}$ released from coal EGUs divided by the amount of electricity generated from coal and woody biomass (EPA 2014a). Constraint (4.10) ensures that $\mathrm{CO}_{2}$ emissions per MWh from state $s$ during time period $t$ do not exceed the allowable upper bound, subject to a userspecified level of robustness.

$$
\sum_{p \in \mathcal{P}} \alpha_{p s} \bar{\delta}_{p}^{t} z_{p}^{t}+\breve{\ell}_{s}^{t} \Gamma_{s}^{t}+\sum_{p \in \mathcal{P}_{s}^{t}} \breve{m}_{p s}^{t} \leq \phi_{s}^{t} \sum_{p \in \mathcal{P}} \alpha_{p s}\left(z_{p}^{t}+y_{p}^{t}\right) \quad \forall t \in \mathcal{T}, \quad \forall s \in \mathcal{S}
$$

The final two terms on the left-hand-side of the inequality, which account for robustness, are based on the RO formulation of Bertsimas \& Sim (2003). The constraint coefficients that were assumed to be uncertain are the $\mathrm{CO}_{2}$ emission rates at each power plant. The uncertain plant $\mathrm{CO}_{2}$ emission rate is denoted $\delta_{p}^{t}$; we assume that this parameter is bounded and varies symmetrically inside the interval $\left[\bar{\delta}_{p}^{t}-\hat{\delta}_{p}^{t}, \bar{\delta}_{p}^{t}+\hat{\delta}_{p}^{t}\right]$ and that these variations occur independently. Here the nominal value of the $\mathrm{CO}_{2}$ emission rate is denoted as $\bar{\delta}_{p}^{t}$, and the maximum-allowable deviation (halfwidth) of potential parameter variations is denoted as $\hat{\delta}_{p}^{t}$.

Let $P_{s}^{t}=\left\{p \mid \hat{\delta}_{p}^{t}>0, \alpha_{p s}=1\right\}$, denoting, for power plants located in state $s$, the subset of $\mathrm{CO}_{2}$ emission rate parameters that can potentially deviate from their nominal values in year $t$. Based on the formulation of Bertsimas \& Sim (2004), we utilize input parameter $\Gamma_{s}^{t}$ to allow the user to control the level of robustness in the emissions constraint associated with time period $t$ and state $s$. When the user sets $\Gamma_{s}^{t}=0$, it means that no parameters are allowed to deviate from their nominal values; what remains is a non-robust model. When the user sets $\Gamma_{s}^{t}=\left|P_{s}^{t}\right|$, all uncertain emissions 
parameters are allowed to deviate from their nominal values; in this case the optimal solutions that are obtained are based on the worst-case scenario. For an intermediate value of $\Gamma_{s}^{t}$ between these two endpoints, the terms $\breve{\ell}_{s}^{t} \Gamma_{s}^{t}+\sum_{p \in \mathcal{P}_{s}^{t}} \breve{m}_{p s}^{t}$ in constraint (4.10) compute the additional emissions (above the nominal levels) associated with the $\Gamma_{s}^{t}$ worst-case-impact values of decision variable $z_{p}^{t}$.

To control for the desired level of robustness $\Gamma_{s}^{t}$ in emissions constraint (4.10), it is necessary to add constraints (4.11) - (4.15), which are presented in below.

$$
\begin{array}{rc}
\breve{\ell}_{s}^{t}+\breve{m}_{p s}^{t} \geq \hat{\delta}_{p}^{t} \breve{n}_{p}^{t} & \forall t \in \mathcal{T}, \quad \forall s \in \mathcal{S}, \quad \forall p \in \mathcal{P}_{s}^{t} \\
\breve{m}_{p s}^{t} \geq 0 & \forall t \in \mathcal{T}, \quad \forall s \in \mathcal{S}, \quad \forall p \in \mathcal{P}_{s}^{t} \\
\breve{n}_{p}^{t} \geq 0 & \forall t \in \mathcal{T}, \quad \forall p \in \mathcal{P} \\
\breve{\ell}_{s}^{t} \geq 0 & \forall t \in \mathcal{T}, \quad \forall s \in \mathcal{S} \\
-\breve{n}_{p}^{t} \leq z_{p}^{t} \leq \breve{n}_{p}^{t} & \forall t \in \mathcal{T}, \quad \forall p \in \mathcal{P}
\end{array}
$$

\subsubsection{Robust counterpart of MILP}

In the compact form, the RO MILP objective function and constraints are as follows. As discussed in section 4.1, constraints (4.16)-(4.41), which do not contain uncertain data parameters, remain unchanged from the MILP formulation of previous chapter. 


$$
\begin{array}{r}
\min \sum_{t \in \mathcal{T}} \sum_{p \in \mathcal{P}} \sigma_{p}^{t} x_{p}^{t}+\sum_{t \in \mathcal{T}} \sum_{p \in \mathcal{P}} \bar{\gamma}_{p}^{t} w_{p}^{t}+\bar{\pi}_{p}^{t} \bar{g}_{p}^{t}+\sum_{t \in \mathcal{T}} \sum_{p \in \mathcal{P}} \sum_{r \in \mathcal{R}} \pi_{p r}^{t} g_{p r}^{t}+\sum_{t \in \mathcal{T}} \sum_{p \in \mathcal{P}} \lambda_{p}^{t} y_{p}^{t}+ \\
\sum_{t \in \mathcal{T}} \sum_{p \in \mathcal{P}} \bar{\rho}_{p}^{t} z_{p}^{t}+\ell \Gamma_{0}+\sum_{(t, p) \in \tilde{\mathcal{P}}} \tilde{m}_{p}^{t}+\sum_{(t, p) \in \mathcal{P}} \dot{m}_{p}^{t}
\end{array}
$$

$$
\sum_{p \in \mathcal{P}} \alpha_{p s} \bar{\delta}_{p}^{t} z_{p}^{t}+\breve{\ell}_{s}^{t} \Gamma_{s}^{t}+\sum_{p \in \mathcal{P}_{s}^{t}} \breve{m}_{p s}^{t} \leq \phi_{s}^{t} \sum_{p \in \mathcal{P}} \alpha_{p s}\left(z_{p}^{t}+y_{p}^{t}\right) \quad \forall t \in \mathcal{T}, \quad \forall s \in \mathcal{S}
$$

$$
\begin{aligned}
v_{p}^{t}=\frac{y_{p}^{t}}{\xi} & \forall t \in \mathcal{T}, \quad \forall p \in \mathcal{P} \\
\sum_{p \in \mathcal{P}} \alpha_{p s}\left(y_{p}^{t}+z_{p}^{t}\right) \geq \beta_{s}^{t}+\chi_{s}^{t} & \forall t \in \mathcal{T}, \quad \forall s \in \mathcal{S} \\
y_{p}^{t}+z_{p}^{t} \leq \psi_{p} & \forall t \in \mathcal{T}, \quad \forall p \in \mathcal{P} \\
\sum_{p \in \mathcal{P}} \alpha_{p s} y_{p}^{t} \geq \chi_{s}^{t} & \forall t \in \mathcal{T}, \quad \forall s \in \mathcal{S}
\end{aligned}
$$

$$
\begin{array}{cc}
\sum_{t \in \mathcal{T}} x_{p}^{t} \leq 1 & \forall p \in \mathcal{P} \\
\sum_{t \in \mathcal{T}} w_{p}^{t} \leq 1 & \forall p \in \mathcal{P} \\
w_{p}^{t} \leq \sum_{t^{\prime} \leq t} x_{p}^{t^{\prime}} & \forall t \in \mathcal{T}, \quad \forall p \in \mathcal{P} \\
y_{p}^{t}=a_{p}^{t}+b_{p}^{t} & \forall t \in \mathcal{T}, \quad \forall p \in \mathcal{P}
\end{array}
$$




$$
\begin{aligned}
a_{p}^{t} \leq M\left(\sum_{t^{\prime} \leq t} x_{p}^{t^{\prime}}\right) & \forall t \in \mathcal{T}, \quad \forall p \in \mathcal{P} \\
a_{p}^{t} \leq M\left(1-\sum_{t^{\prime} \leq t} w_{p}^{t^{\prime}}\right) & \forall t \in \mathcal{T}, \quad \forall p \in \mathcal{P} \\
b_{p}^{t} \leq M\left(\sum_{t^{\prime} \leq t} w_{p}^{t^{\prime}}\right) & \forall t \in \mathcal{T}, \quad \forall p \in \mathcal{P} \\
c_{p}^{t} \leq M\left(1-\sum_{t^{\prime} \leq t} x_{p}^{t^{\prime}}\right) & \forall t \in \mathcal{T}, \quad \forall p \in \mathcal{P} \\
d_{p}^{t} \leq M\left(\sum_{t^{\prime} \leq t} w_{p}^{t^{\prime}}\right) & \forall t \in \mathcal{T}, \quad \forall p \in \mathcal{P} \\
e_{p}^{t} \leq M\left(\sum_{t^{\prime} \leq t} x_{p}^{t^{\prime}}\right) & \forall t \in \mathcal{T}, \quad \forall p \in \mathcal{P} \\
e_{p}^{t} \leq M\left(1-\sum_{t^{\prime} \leq t} w_{p}^{t^{\prime}}\right) & \forall t \in \mathcal{T}, \quad \forall p \in \mathcal{P} \\
\mu_{s} \leq \frac{b_{p}^{t}+e_{p}^{t}+f_{p}^{t}}{b_{p}^{t}+e_{p}^{t}+f_{p}^{t}+z_{p}^{t}} \leq \eta_{s} & \\
f_{p}^{t} \leq M\left(1-\sum_{t^{\prime} \leq t} x_{p}^{t^{\prime}}\right) & \forall t \in \mathcal{T}, \quad \forall p \in \mathcal{P} \\
& \\
& \\
& \\
& \\
&
\end{aligned}
$$




$$
\begin{array}{rlrl}
v_{p}^{t}=\sum_{r \in \mathcal{R}} \theta_{p r}^{t} g_{p r}^{t} & \forall t \in \mathcal{T} & \forall p \in \mathcal{P} \\
\bar{g}_{p}^{t}+\sum_{r \in \mathcal{R}} g_{p r}^{t}=1 & \forall t \in \mathcal{T} & \forall p \in \mathcal{P} \\
\sum_{r \in \mathcal{R}} h_{p r}^{t}=1 & \forall t \in \mathcal{T} & \forall p \in \mathcal{P} \\
\bar{g}_{p}^{t} \leq h_{p 1}^{t} & \forall t \in \mathcal{T}, \quad \forall p \in \mathcal{P}
\end{array}
$$

$$
\begin{array}{rr}
g_{p r}^{t} \leq h_{p r}^{t}+h_{p(r+1)}^{t} \quad r=1 \ldots(|\mathcal{R}|-1), & \forall t \in \mathcal{T}, \quad \forall p \in \mathcal{P} \\
& g_{p|\mathcal{R}|}^{t} \leq h_{p|\mathcal{R}|}^{t} \quad \forall t \in \mathcal{T}, \quad \forall p \in \mathcal{P}
\end{array}
$$$$
x_{p}^{t}, w_{p}^{t}, h_{p r}^{t} \in\{0,1\} \quad \forall t \in \mathcal{T}, \quad \forall p \in \mathcal{P}, \quad \forall r \in \mathcal{R}
$$$$
v_{p}^{t}, y_{p}^{t}, z_{p}^{t}, a_{p}^{t}, b_{p}^{t}, c_{p}^{t}, d_{p}^{t}, e_{p}^{t}, f_{p}^{t}, g_{p r}^{t}, \bar{g}_{p}^{t} \geq 0 \quad \forall t \in \mathcal{T}, \quad \forall p \in \mathcal{P}, \quad \forall r \in \mathcal{R}
$$

$\ell+\tilde{m}_{p}^{t} \geq \hat{\gamma}_{p}^{t} \tilde{n}_{p}^{t} \quad \forall(t, p) \in \tilde{\mathcal{P}}$

$\ell+\dot{m}_{p}^{t} \geq \hat{\rho}_{p}^{t} \dot{n}_{p}^{t} \quad \forall(t, p) \in \dot{\mathcal{P}}$

$\tilde{m}_{p}^{t} \geq 0 \quad \forall(t, p) \in \tilde{\mathcal{P}}$

$\dot{m}_{p}^{t} \geq 0 \quad \forall(t, p) \in \dot{\mathcal{P}}$

$\tilde{n}_{p}^{t}, \dot{n}_{p}^{t} \geq 0 \quad \forall t \in \mathcal{T}, \quad \forall p \in \mathcal{P}$

$\ell \geq 0$

$-\tilde{n}_{p}^{t} \leq w_{p}^{t} \leq \tilde{n}_{p}^{t} \quad \forall t \in \mathcal{T}, \quad \forall p \in \mathcal{P}$

$-\dot{n}_{p}^{t} \leq z_{p}^{t} \leq \dot{n}_{p}^{t} \quad \forall t \in \mathcal{T}, \quad \forall p \in \mathcal{P}$

$-\tilde{n}_{p}^{t} \leq w_{p}^{t} \leq \tilde{n}_{p}^{t} \quad \forall t \in \mathcal{T}, \quad \forall p \in \mathcal{P}$ 


$$
\begin{aligned}
& \breve{m}_{p s}^{t} \geq 0 \quad \forall t \in \mathcal{T}, \quad \forall s \in \mathcal{S}, \quad \forall p \in \mathcal{P}_{s}^{t} \\
& \breve{n}_{p}^{t} \geq 0 \quad \forall t \in \mathcal{T}, \quad \forall p \in \mathcal{P} \\
& \breve{\ell}_{s}^{t} \geq 0 \quad \forall t \in \mathcal{T}, \quad \forall s \in \mathcal{S} \\
& -\breve{n}_{p}^{t} \leq z_{p}^{t} \leq \breve{n}_{p}^{t} \quad \forall t \in \mathcal{T}, \quad \forall p \in \mathcal{P}
\end{aligned}
$$

\subsection{Data Integration for Uncertain Data Parame- ters}

We illustrate our RO model with application to the set of 18 Northern US states presented in Figure 1.1. The map in Figure 4.1 indicates the geographic location of each candidate power plant included in this chapter's analysis. These plants were selected for inclusion in this analysis based on plant characteristics such as the use of stoker, cyclone and fluidized bed boilers on their premises which can relatively easily incorporate the co-firing of woody biomass into the existing facilities (Aguilar et al. 2012); the figure further identifies candidate power plants that are currently primarily powered by biomass.

We estimated the total electricity requirements in each state from coal and woody biomass in each year over the interval 2022-2030 utilizing EIA and EPA data (EPA 2014a). For those data parameters that were not assumed to be uncertain in this RO model, the data collection procedure utilized in previous chapter was implemented. The remainder of this section focuses on the data integration procedure utilized for the three parameters that were assumed to be uncertain in our RO model. 


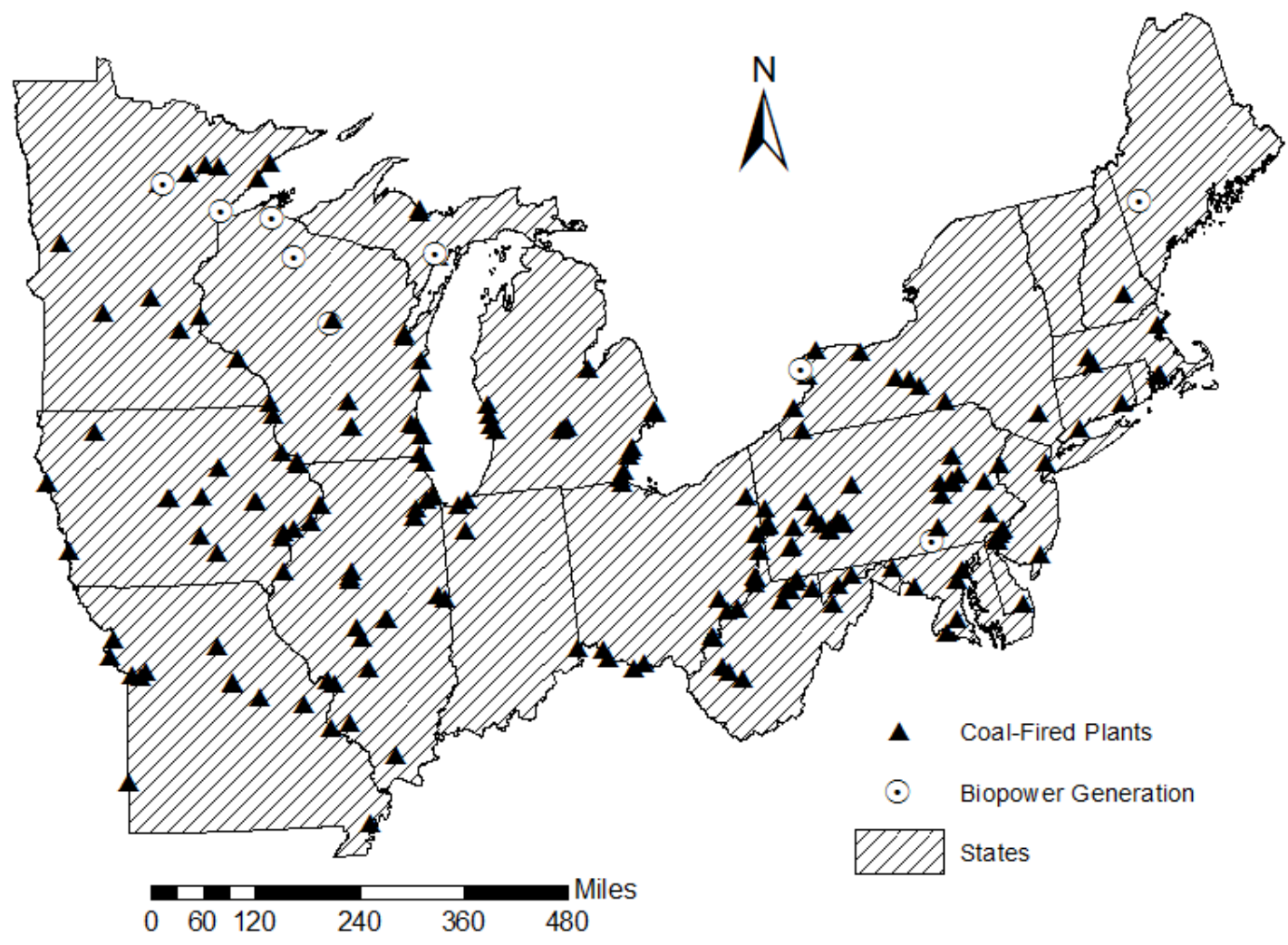

Figure 4.1: Locations of candidate power plants

\subsubsection{Coal electricity generation cost}

The uncertain coal electricity generation cost per MWh includes the sum of the coal procurement price and the plant O\&M cost. For procurement, we obtained statespecific data for the coal price per short ton from the EIA annual reports over the period 2004-2014 (EIA 2013). As demonstrated in Figure 4.2, there is considerable year-to-year variability in the price for states such as New Hampshire or West Virginia. Moreover, for some states, such as Pennsylvania, data was not disclosed for certain years. We estimated the nominal value for each state's coal price per short ton as the midpoint between the state's maximum and minimum observed coal price, with the maximum-allowable deviation for each state equal to one-half of the difference 
of the maximum and minimum values. Table 4.1 presents these procurement price estimates for each state included in this analysis.

Table 4.1: The nominal value and maximum allowable deviation (MAD) for coal price per ton and for electricity generation cost

\begin{tabular}{lllll}
\hline & \multicolumn{2}{c}{ Coal Price(\$/ton) } & \multicolumn{2}{c}{ Coal electricity generation cost(\$/MWh) } \\
\cline { 2 - 5 } States & Nominal value & MAD & Nominal value & MAD \\
\hline MO & 25.75 & 9.44 & 17.00 & 5.78 \\
IA & 22.19 & 6.63 & 15.13 & 4.30 \\
MN & 27.23 & 8.45 & 17.78 & 5.26 \\
WI & 33.16 & 12.30 & 20.89 & 7.28 \\
CT & 72.38 & 7.99 & 41.49 & 5.02 \\
DE & 92.71 & 8.71 & 52.17 & 5.39 \\
IL & 27.85 & 6.42 & 18.11 & 4.19 \\
IN & 39.19 & 13.49 & 24.06 & 7.90 \\
MA & 67.71 & 19.47 & 39.04 & 11.04 \\
MD & 83.18 & 8.90 & 47.17 & 5.50 \\
ME & 130.5 & 32.14 & 71.28 & 17.70 \\
MI & 41.20 & 14.05 & 25.12 & 8.20 \\
NH & 80.75 & 27.58 & 45.89 & 15.31 \\
NJ & 82.83 & 22.95 & 46.98 & 12.87 \\
NY & 56.02 & 14.83 & 32.90 & 8.61 \\
OH & 44.94 & 12.95 & 27.08 & 7.62 \\
PA & 44.09 & 12.28 & 26.64 & 7.27 \\
WV & 47.68 & 13.55 & 28.52 & 7.93 \\
\hline & & & & \\
\hline
\end{tabular}

For O\&M costs, we utilized data from a National Renewable Energy Laboratory report (Tidball et al. 2010). This report presents estimates from four different organizations for power plant O\&M costs associated with electricity generation from coal. We assumed that the nominal O\&M value was equal to the mean $(\$ 30.48 / \mathrm{kW} / \mathrm{yr})$ across these four samples, which is equivalent to $\$ 3.48$ per MWh. In order to calculate the maximum-allowable deviation value, we computed a $99.9 \%$ confidence interval based on this sample mean and standard deviation, obtaining a halfwidth value of 


\section{$\$ 0.82$ per MWh.}

Table 4.1 also presents the estimated nominal $\left(\bar{\rho}_{p}^{t}\right)$ and maximum-allowable deviation $\left(\hat{\rho}_{p}^{t}\right)$ values for the total coal generation cost per MWh for each state, computed as follows. For power plants in state $s$, the nominal value is equal to the state $s$ nominal coal procurement price plus $\$ 3.48$. The maximum-allowable deviation value is equal to the maximum-allowable deviation for state $s$ coal procurement price plus $\$ 0.82$ Note that it is assumed that the coal electricity generation cost is equal across all power plants in a given state.

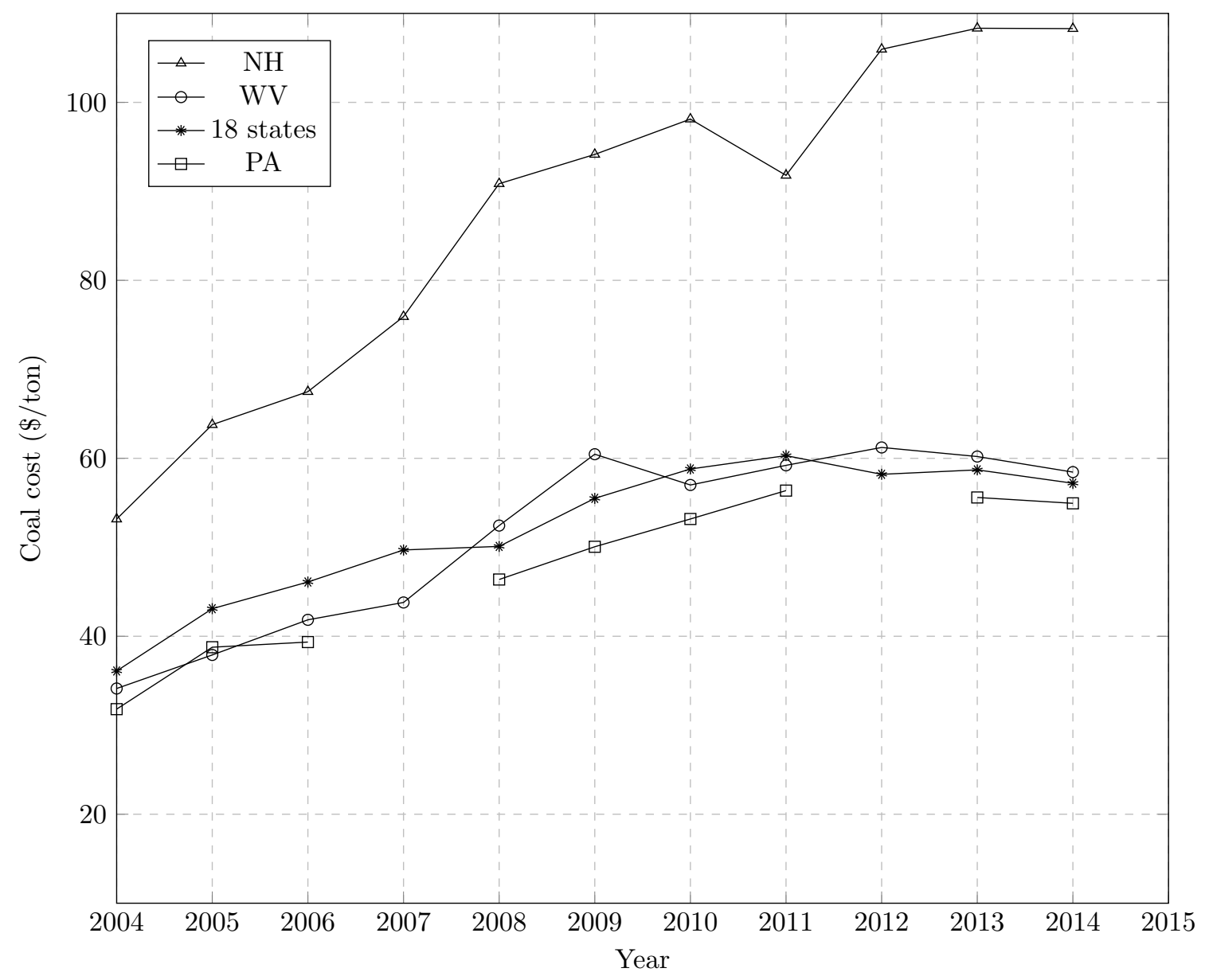

Figure 4.2: The price of coal per ton during 2004-2015 


\subsection{2 $\mathrm{CO}_{2}$ emission rate}

We used 2007, 2009, 2010, and 2012 emission data for each plant (EPA 2014b) to estimate the nominal value and the maximum-allowable deviation of the uncertain $\mathrm{CO}_{2}$ emission rate parameters. Table 4.2 presents, for each state, the mean emission rate across all power plants included in this study (weighted by the amount of electricity generated at each plant during the observed years). Observe that these values fluctuate considerably over the four years that were considered. We estimated the nominal value $\left(\bar{\delta}_{p}^{t}\right)$ for each plant as the midpoint between that plant's maximum and minimum observed values, with the maximum-allowable deviation $\left(\hat{\delta}_{p}^{t}\right)$ for each plant equal to one-half of the difference of these maximum and minimum values.

Table 4.2: Weighted mean emission rate, by state, across power plants included in this analysis

\begin{tabular}{lllllcl}
\hline & \multicolumn{3}{c}{ Years } & & & \\
States & 2007 & 2009 & 2010 & 2012 & Nominal Value(lb/MWh) & Range(lb/MWh) \\
\hline MO & $2,037.6$ & $2,142.1$ & $2,156.5$ & $2,045.2$ & 2,097 & 59.4 \\
IA & $2,217.3$ & $2,214.7$ & $2,215.6$ & $2,174.2$ & $2,195.7$ & 21.5 \\
MN & $2,285.7$ & $2,279.7$ & $2,229.8$ & $2,258.4$ & $2,257.8$ & 27.9 \\
WI & $2,294.6$ & $2,246.8$ & $2,266.7$ & $2,286.4$ & $2,270.7$ & 23.9 \\
CT & $2,070.3$ & $1,976.3$ & $1,996.1$ & $3,146.6$ & $2,561.4$ & 585.1 \\
DE & $2,180.7$ & $2,025.1$ & $1,990.9$ & $1,792.6$ & $1,986.7$ & 194.1 \\
IL & $2,213.5$ & $2,224.3$ & $2,208.4$ & $2,238.3$ & $2,223.4$ & 14.9 \\
IN & $2,313.5$ & $2,402.7$ & $2,347.8$ & $2,403.4$ & $2,358.5$ & 44.9 \\
MA & $1,946.9$ & $1,979.4$ & $1,984.5$ & $2,079.1$ & 2,013 & 66.1 \\
MD & $1,989.3$ & $2,010.6$ & $2,150.2$ & $2,037.6$ & $2,069.7$ & 80.4 \\
ME & 321.1 & 259.4 & 245.8 & 215.7 & 268.4 & 52.7 \\
MI & $2,133.3$ & $2,141.3$ & $2,159.6$ & $2,219.1$ & $2,176.2$ & 42.9 \\
NH & $2,266.9$ & $2,155.5$ & $2,110.3$ & $2,353.3$ & $2,231.8$ & 121.5 \\
NJ & $2,146.7$ & $2,185.2$ & $2,077.9$ & $1,750.3$ & $1,967.8$ & 217.5 \\
NY & $2,038.7$ & $2,071.2$ & $2,067.4$ & $2,089.3$ & 2,064 & 25.3 \\
OH & $1,992.3$ & $2,050.7$ & $2,077.3$ & $2,110.6$ & $2,051.4$ & 59.1 \\
PA & $1,985.2$ & $2,023.3$ & $2,065.5$ & 2,079 & $2,032.1$ & 46.9 \\
WV & $1,992.7$ & $2,079.9$ & $2,026.8$ & $2,055.2$ & $2,036.3$ & 43.6 \\
\hline
\end{tabular}




\subsubsection{Boiler upgrading cost}

Consistent with the assumptions of previous chapter, we assume that in order for a power plant to generate more than $10 \%$ of its total annual MWh from woody biomass, the plant must upgrade to a new boiler system specifically designed to burn woody biomass (a plant can generate up to $10 \%$ of its total annual MWh from woody biomass with only an upgrade to the plant's MHS). Based on information provided by University of Missouri, which recently upgraded its coal-fired cogeneration plant to co-fire woody biomass, we estimated a baseline boiler upgrading cost of $\$ 50$ million for a boiler with a nameplate capacity of 40MWh. Nominal boiler upgrade costs for other power plants $\left(\bar{\gamma}_{p}^{t}\right)$ were assumed to vary linearly from this baseline boiler upgrade cost with respect to each plant's nameplate generation capacity. In discussion with a vendor who performs such facility upgrades, it was indicated that the boiler installation costs are site-specific, with an estimate that the installation costs for a new boiler system could vary by up $30 \%$ of its nominal cost; thus we assume $\left(\hat{\gamma}_{p}^{t}=0.3 \bar{\gamma}_{p}^{t}\right)$.

\subsubsection{The demand for RE generation}

We replaced equation (3.30) with new equation (4.42) to calculate the renewable energy demand target for each state in this 18-state example. In this new equation, $\wp_{s}^{t}$ represents the total electricity generation capacity of state $s$ during time period $t$ across the set of power plants included in this study. $\hbar_{s}^{t}$ is the interim target percentage of state's $s$ total energy generation from renewable sources, excluding hydropower, during time period $t$ (AEO 2013). Note that for the final year of 2030, the target percentage is different from the interim years 2022-2029 for each state. 


$$
\chi_{s}^{t}=\wp_{s}^{t} * \hbar_{s}^{t}
$$

\subsection{RO - Marginal Cost Integrated Approach}

In computational experience, the robust counterpart of the MILP model presented in Section 4.1 was not able to be solved within a reasonable time when all eighteen states were considered simultaneously. Therefore, we extended the marginal cost integrated approach (MCIA) of previous chapter to accommodate an environment with data parameter uncertainty; we refer to this new modeling approach as Robust Optimization - MCIA (ROMCIA). Figure 4.3 presents a schematic overview of the ROMCIA method. In ROMCIA, the RO MILP model of Section 4.1 is solved many times for each individual state to generate a robust marginal cost table (ROMCT) for that state. These ROMCT can then be used in another optimization model to efficiently solve multi-state instances.

\subsubsection{Generating robust marginal cost tables}

An ROMCT contains three dimensions: demand (electricity generation) level, $\mathrm{CO}_{2}$ emission rate, and emission robustness level. Each element of the ROMCT corresponds to a single run of the RO MILP model, solved at predetermined input values of $\beta_{s}^{t}+\chi_{s}^{t}$ (demand level), $\phi_{s}^{t}$ (maximum-allowable $\mathrm{CO}_{2}$ emission rate), and $\Gamma_{s}^{t}$ (emission constraint robustness level). Within each ROMCT, the demand level varies

from the state's minimum renewable energy generation level $\left(\chi_{s}^{t}\right)$ to the maximum 


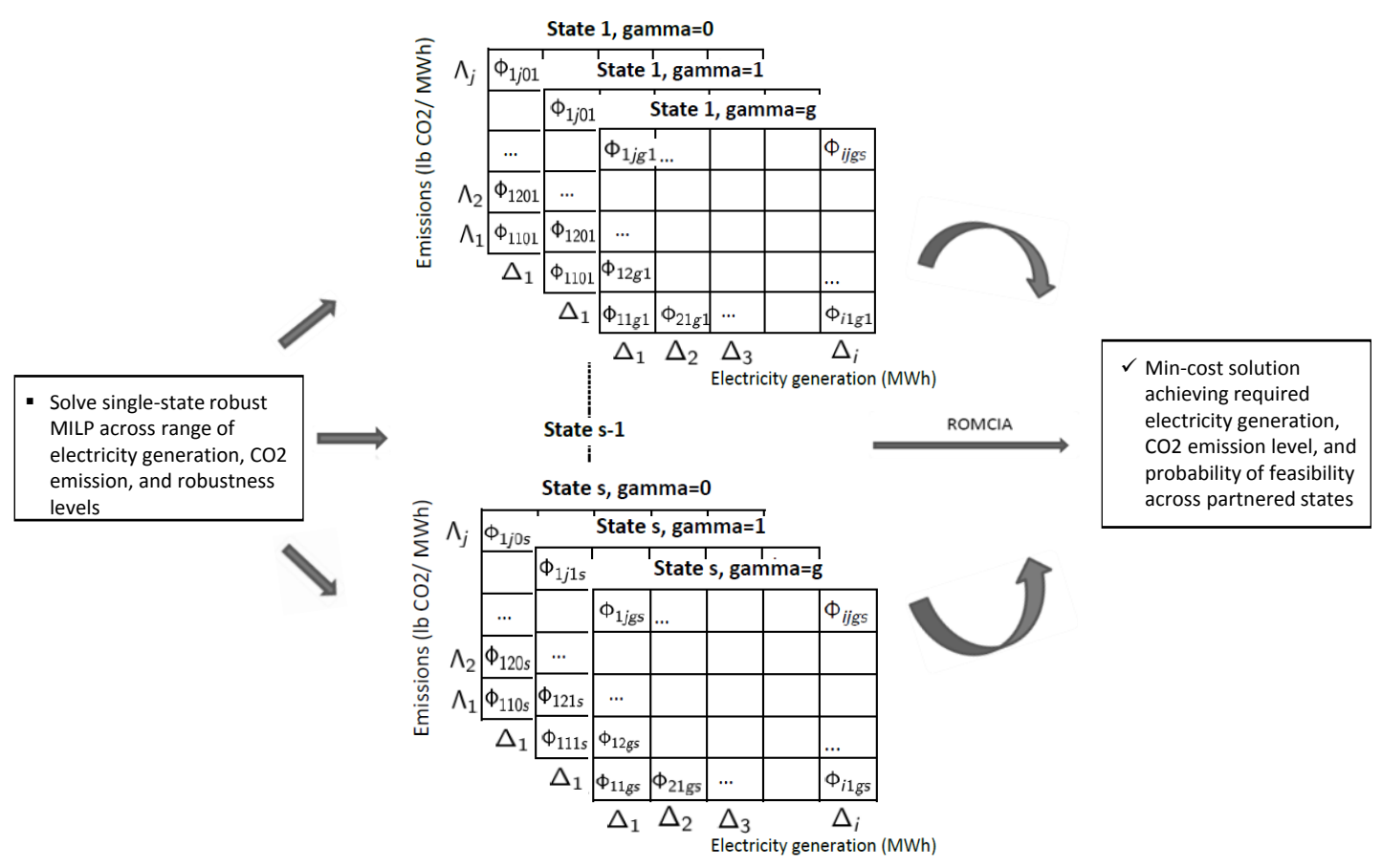

Figure 4.3: ROMCIA modeling approach.

electricity generation capacity across the set of included power plants for that state, across a set of fixed step sizes. The emission rate within an ROMCT varies from the state's minimum-achievable emission rate, depending on the available woody biomass resource and the projected demand, to the state's current emission rate (the weighted average current emission rate across the set of power plants from that state that are included in this study), increasing by increments of $10 \mathrm{lb} / \mathrm{MWh}$. The robustness level, defined in constraint (4.10), varies across all integer values of $\Gamma_{s}^{t}$ ranging from a minimum of 0 to a maximum of $\left|P_{s}^{t}\right|$; this maximum value is equal to the total number of power plants included in our analysis from state $s$. Table 4.3 presents the total computational run time to generate the ROMCT for each state, where the number of model runs corresponds to the number of ROMCT elements for that state (i.e., the number of combinations of $\left(\beta_{s}^{t}+\chi_{s}^{t}, \phi_{s}^{t}, \Gamma_{s}^{t}\right)$ that were solved). These model 
runs were performed using an Intel Core i7-4710MQ (2.50 GHz) processor and 8 GB RAM, with the RO MILP implemented using Python (version 3.2.5) (Python Software Foundation 2016) and solved by the Gurobi solver (Gurobi Optimization 2016).

Table 4.3: Computational run time to generate each ROMCT

\begin{tabular}{lllll}
\hline State & $\begin{array}{l}\text { Number of } \\
\text { power plants }\end{array}$ & $\begin{array}{l}\text { Number of } \\
\text { scenarios }\end{array}$ & $\begin{array}{l}\text { Total run } \\
\text { time(hrs) }\end{array}$ & $\begin{array}{l}\text { Total run } \\
\text { per scenario(sec) }\end{array}$ \\
\hline MO & 14 & 28,830 & 20.13 & 2.5 \\
IA & 23 & 25,584 & 17.68 & 2.5 \\
MN & 13 & 15,120 & 8.13 & 1.9 \\
WI & 20 & 15,876 & 17.95 & 4.1 \\
CT & 2 & 660 & 0.14 & 0.7 \\
DE & 2 & 420 & 0.08 & 0.7 \\
IL & 21 & 43,956 & 31.15 & 2.6 \\
IN & 4 & 2,210 & 0.45 & 0.7 \\
MA & 5 & 1,536 & 0.33 & 0.8 \\
MD & 9 & 14,030 & 8.09 & 2.1 \\
ME & 1 & 120 & 0.24 & 7.2 \\
MI & 21 & 35,464 & 95.57 & 9.7 \\
NH & 1 & 452 & 0.08 & 0.6 \\
NJ & 5 & 3,000 & 0.81 & 1.0 \\
NY & 11 & 9,672 & 7.25 & 2.7 \\
OH & 10 & 24,640 & 27.83 & 4.1 \\
PA & 30 & 20,088 & 301.27 & 54.0 \\
WV & 17 & 49,248 & 253.87 & 18.6 \\
\hline
\end{tabular}

Observe that we did not vary the robust objective function parameter $\Gamma_{0}$ within the ROMCTs. During preliminary computational testing, we evaluated the impact of the robust parameters $\Gamma_{0}$ and $\Gamma_{s}^{t}$ on the resultant optimal solutions' level of renewable energy generation and nominal objective function (i.e., the value of the objective function excluding the robustness terms in function (4.1)). As an example, consider the ROMCT corresponding to Missouri, at a demand level equal to Missouri's total 
independent electricity generation requirements, at an emission rate of $2025 \mathrm{lb} / \mathrm{MWh}$. We ran the RO MILP model across all possible integer values of $\Gamma_{0}$ and $\Gamma_{s}^{t}$; Table 4.4 presents the percent renewable energy generation for a subset of the considered cases. While MO had 14 candidate power plants in this analysis, the model was not able to obtain a feasible solution at this emission level for values of $\Gamma_{s}^{t}>9$; at this level of electricity generation the emission rate of $2025 \mathrm{lb} / \mathrm{MWh}$ (which is considerably lower than these 14 plant's nominal historical level of $2097 \mathrm{lb} / \mathrm{MWh}$ ) cannot be achieved if more than nine plants could have their emission levels vary to their worst-case-impact levels. Observe in Table 4.4 that the percent of renewable energy generation increases as $\Gamma_{s}^{t}$ increases. For example, at $\Gamma_{s}^{t}=9$, in order to guard against the instance when nine power plants could have their emission parameters take their worst-case-impact values, the percentage of electricity produced from woody biomass increases from $2.52 \%$ to $5.48 \%$. However, Table 4.4 also makes apparent that the percent of renewable energy generation is not significantly impacted as $\Gamma_{0}$ is increased from its minimum value of 0 to its maximum value of $|\tilde{P}|+|\dot{P}|=252$, for constant $\Gamma_{s}^{t}$. Similarly, we found the nominal objective function value to increase as $\Gamma_{s}^{t}$ increases, but that for a constant $\Gamma_{s}^{t}$ this value varies by at most $0.1 \%$ as $\Gamma_{0}$ is increased. Therefore, in order to reduce the dimensionality of the ROMCTs, and thus reduce the number of RO MILP runs necessary to populate the ROMCTs, we fixed $\Gamma_{0}$ to a single intermediate value for each state (e.g., for Missouri, we set set $\left.\Gamma_{0}=140\right)$. 
Table 4.4: Percentage of electricity generated from woody biomass in Missouri

\begin{tabular}{c|cccccccccc}
\hline \multicolumn{10}{c}{$\Gamma_{s}^{t}$} \\
\cline { 2 - 12 }$\Gamma_{0}$ & 0 & 1 & 2 & 3 & 4 & 5 & 6 & 7 & 8 & 9 \\
\hline 0 & 2.52 & 2.97 & 3.72 & 4.02 & 4.43 & 4.97 & 5.19 & 5.32 & 5.45 & 5.48 \\
1 & 2.52 & 2.97 & 3.72 & 4.02 & 4.60 & 4.97 & 5.19 & 5.32 & 5.45 & 5.48 \\
140 & 2.52 & 2.97 & 3.72 & 4.36 & 4.43 & 4.97 & 5.19 & 5.32 & 5.44 & 5.48 \\
252 & 2.52 & 2.97 & 3.72 & 4.36 & 4.60 & 4.97 & 5.19 & 5.32 & 5.45 & 5.48 \\
\hline
\end{tabular}

\subsubsection{ROMCIA optimization model}

The ROMCIA optimization model for determining multi-state collaborations is represented using the following new sets and indices, data parameters, and decision variables:

\section{Sets and indices}

- $\mathcal{I}$ : set of potential electricity generation levels, indexed by $i$

- $\mathcal{J}$ : set of potential $\mathrm{CO}_{2}$ emission levels, indexed by $j$

- $\mathcal{G}$ : set of state emission constraint feasibility (robustness) levels, indexed by $g$

- $\mathcal{K}$ : set of step sizes used by linear approximation of objective function, indexed by $k$

- $\mathcal{S}$ : set of all states considered, indexed by $s$ (defined previously in Section 4.1)

\section{Data parameters}

- $\Phi_{i j g s}$ : cost to achieve electricity generation level $i$ at $\mathrm{CO}_{2}$ emission level $j$ with robustness level $g$ in state $s$, across all years

- $\Delta_{i s}$ : MWh corresponding to electricity generation level $i$ in state $s$

- $\Lambda_{j s}$ : $\mathrm{CO}_{2}$ emission rate, in pounds/MWh, corresponding to emission level $j$ in state $s$ 
- $\Xi_{s}$ : total MWh of required electricity generation in state $s$

- $\Omega$ : maximum allowable aggregate multi-state $\mathrm{CO}_{2}$ emission rate

- $\Upsilon_{q}$ : set of potential objective values considered for linear approximation, indexed by $q$

- $\Theta_{g s}:$ probability of the solution being feasible with respect to the emission constraint at robustness level $g$ for state $s$

- $\Psi$ : lower bound of aggregate feasibility for the multi-state collaboration

\section{Decision variables}

- $u_{i j g s}= \begin{cases}1 & \text { if electricity generation level } i \text { is achieved at } \mathrm{CO}_{2} \text { emission level } j \\ & \text { with robustness level } g \text { in state } s \\ 0 & \text { otherwise }\end{cases}$
- $o_{k}= \begin{cases}1 & \text { if objective function linear approximation segment } k \text { is selected } \\ 0 & \text { otherwise }\end{cases}$

The MCIA objective function and constraints are as follows.

Term (4.43) is the objective function, which aims to minimize the aggregate cost per MWh generated across all states selected for partnership. Observe that this function is nonlinear.

$$
\min \frac{\sum_{i \in \mathcal{I}} \sum_{j \in \mathcal{J}} \sum_{g \in \mathcal{G}} \sum_{s \in \mathcal{S}} \Phi_{i j g s} u_{i j g s}}{\sum_{i \in \mathcal{I}} \sum_{j \in \mathcal{J}} \sum_{g \in \mathcal{G}} \sum_{s \in \mathcal{S}} \Delta_{i s} u_{i j g s}}
$$

Constraint (4.44) ensures that the total electricity generation satisfies the aggre- 
gate demand over all states that are selected for partnerships.

$$
\sum_{i \in \mathcal{I}} \sum_{j \in \mathcal{J}} \sum_{g \in \mathcal{G}} \sum_{s \in \mathcal{S}} \Delta_{i s} u_{i j g s} \geq \sum_{i \in \mathcal{I}} \sum_{j \in \mathcal{J}} \sum_{g \in \mathcal{G}} \sum_{s \in \mathcal{S}} \Xi_{s} u_{i j g s}
$$

Constraint (4.45) ensures that the aggregate $\mathrm{CO}_{2}$ emissions per $\mathrm{MWh}$ do not exceed the multi-state aggregated emission target. We can replace this nonlinear constraint with an equivalent linear constraint by multiplying each side of the inequality by the left-hand-side denominator, which is strictly positive (provided at least one state is included in the partnership).

$$
\frac{\sum_{i \in \mathcal{I}} \sum_{j \in \mathcal{J}} \sum_{g \in \mathcal{G}} \sum_{s \in \mathcal{S}} \Lambda_{j s} \Delta_{i s} u_{i j g s}}{\sum_{i \in \mathcal{I}} \sum_{j \in \mathcal{J}} \sum_{g \in \mathcal{G}} \sum_{s \in \mathcal{S}} \Delta_{i s} u_{i j g s}} \leq \Omega
$$

Constraint (4.46) ensures that at most one electricity generation level, emission level, and robustness level is selected for each state that is selected for partnership.

$$
\sum_{i \in \mathcal{I}} \sum_{j \in \mathcal{J}} \sum_{g \in \mathcal{G}} u_{i j g s} \leq 1, \quad \forall s \in \mathcal{S}
$$

The final constraint in the ROMCIA model, constraint (4.48), was added to capture the probability of achieving the desired aggregate emission rate. As presented in Bertsimas \& Sim (2004), let $z_{p}^{t *}$ and $y_{p}^{t *}$ correspond to the optimal solution of the robust MILP; the probability of infeasibility of constraint (4.10) for an instance of 
$(s, t)$ can then be estimated by inequality (4.47)

$$
\begin{aligned}
& \operatorname{Pr}\left(\sum_{p \in \mathcal{P}} \alpha_{p s} \delta_{p}^{t} z_{p}^{t *}>\phi_{s}^{t} \sum_{p \in \mathcal{P}} \alpha_{p s}\left(z_{p}^{t *}+y_{p}^{t *}\right)\right) \leq \\
&\left.\frac{1}{2^{\aleph}}\left\{(1-ד) \sum_{l=\lfloor\beth\rfloor}^{\aleph}\left(\begin{array}{c}
\aleph \\
l
\end{array}\right)+\right\urcorner \sum_{l=\lfloor\beth]+1}^{\aleph}\left(\begin{array}{c}
\aleph \\
l
\end{array}\right)\right\}
\end{aligned}
$$

where $\aleph=\left|P_{s}^{t}\right|$ (here, the total number of power plants in state $s$ ), $\beth=\frac{\Gamma_{s}^{t}+\aleph}{2}$ and \urcorner$=\beth-\lfloor\beth\rfloor$. Observe that we can use this inequality to compute parameter $\Theta_{g s}$, the probability of feasibility of constraint (4.10), for each value of $\Gamma_{s}^{t}$ that is considered for each state. Constraint (4.48) then provides a lower bound on the feasibility on the partnership satisfying its emissions constraints, assuming independence in feasibility probability across states. Observe that this constraint is nonlinear.

$$
\prod_{s \in \mathcal{S}}\left(\sum_{i \in \mathcal{I}} \sum_{j \in \mathcal{J}} \sum_{g \in \mathcal{G}} u_{i j g s} \Theta_{g s}+\left(1-\sum_{i \in \mathcal{I}} \sum_{j \in \mathcal{J}} \sum_{g \in \mathcal{G}} u_{i j g s}\right)\right) \geq \Psi
$$

\subsubsection{Linearization of terms in ROMCIA model}

In order to represent the ROMCIA as a linear model, we utilize a linear approximation of nonlinear objective function (4.43). Objective (4.43) is replaced with objective (4.49), and new constraints (4.50) - (4.51) are added to the model; $M$ here denotes a large positive constant that is greater than the largest possible total cost:

$$
\min \sum_{k \in \mathcal{K}} \Upsilon_{k} o_{k}
$$




$$
\begin{aligned}
\sum_{i \in \mathcal{I}} \sum_{j \in \mathcal{J}} \sum_{g \in \mathcal{G}} \sum_{s \in \mathcal{S}} \Phi_{i j g s} u_{i j g s} \leq \\
\Upsilon_{k}\left(\sum_{i \in \mathcal{I}} \sum_{j \in \mathcal{J}} \sum_{g \in \mathcal{G}} \sum_{s \in \mathcal{S}} \Xi_{s} u_{i j g s}\right)+M\left(1-o_{k}\right) \quad \forall k \in \mathcal{K}
\end{aligned}
$$

$$
\sum_{k \in \mathcal{K}} o_{k}=1
$$

Partnership feasibility lower bound constraint (4.48) is nonlinear due to the left-handside term containing the product of binary variables $u_{i j g s}$. In order to linearize this constraint, we can take the log of both sides of the constraint, resulting in:

$$
\sum_{s \in \mathcal{S}} \log \left(\sum_{i \in \mathcal{I}} \sum_{j \in \mathcal{J}} \sum_{g \in \mathcal{G}} u_{i j g s} \Theta_{g s}+\left(1-\sum_{i \in \mathcal{I}} \sum_{j \in \mathcal{J}} \sum_{g \in \mathcal{G}} u_{i j g s}\right)\right) \geq \log (\Psi)
$$

We can then rearrange the terms as follows:

$$
\sum_{s \in \mathcal{S}} \log \left(1+\sum_{i \in \mathcal{I}} \sum_{j \in \mathcal{J}} \sum_{g \in \mathcal{G}} u_{i j g s} \Theta_{g s}-\sum_{i \in \mathcal{I}} \sum_{j \in \mathcal{J}} \sum_{g \in \mathcal{G}} u_{i j g s}\right) \geq \log (\Psi)
$$

Since $u_{i j g s}$ is a binary variable, it may only take values 0 or 1 . Recall from constraint (4.46) that, for any state $s, \sum_{i \in \mathcal{I}} \sum_{j \in \mathcal{J}} \sum_{g \in \mathcal{G}} u_{i j g s}$ equals either 0 or 1 . If this sum equals zero for state $s$, then the left-hand-side summation term of constraint (4.53) corresponding to state $s$ must be equal to $\log (1)=0$. Otherwise, this left hand side term must be equal to $\log \left(\Theta_{g s}\right)$ corresponding to the unique $u_{i j g s}$ that is equal to 1 . Thus, constraint 
(4.53) is exactly equivalent to the following linear constraint.

$$
\sum_{i \in \mathcal{I}} \sum_{j \in \mathcal{J}} \sum_{g \in \mathcal{G}} \sum_{s \in \mathcal{S}} u_{i j g s} \log \left(\Theta_{g s}\right) \geq \log (\Psi)
$$

The final ROMCIA model that is utilized in this study consists of objective (4.49) and constraints (4.44), (4.45), (4.46), (4.50), (4.51) and (4.54).

\subsection{Results and Discussion}

Observe that the ROMCIA model allows the efficient frontier of cost-effective partnerships to be automatically generated for any state, since the specific states joining in partnership do not need to be identified in advance. This is an advance over the MCIA model of previous chapter, in addition to the ROMCIA model's ability to address data uncertainty, since the earlier MCIA approach could not automatically identify such efficient frontiers. Consider a situation in which one wants to identify the efficient frontier of potential partnerships for one state, say, Missouri. In previous chapter, we had to examine every potential subset of partnering states that includes Missouri, solving their MCIA model across the range of achievable aggregate emissions levels for each subset, and then identify the points lying on the efficient frontier. For the example considered in this study, Missouri has 17 potential partner states, corresponding to a total of $2^{17}=131,072$ potential subsets of partnering states that would need to examined under the previous MCIA approach. The ROMCIA model, however, allows this efficient frontier to be identified automatically.

We ran the ROMCIA model for different feasibility $(\Psi)$ values to determine op- 
timal partnering strategies for one state (Missouri). Figures 4.4, 4.5 and 4.6 present the efficient frontiers associated with $\Psi=0 \%, 50 \%$ and $90 \%$, respectively. Figure 4.7 presents these efficient frontiers, along with that for $\Psi=75 \%$, on a single graph.

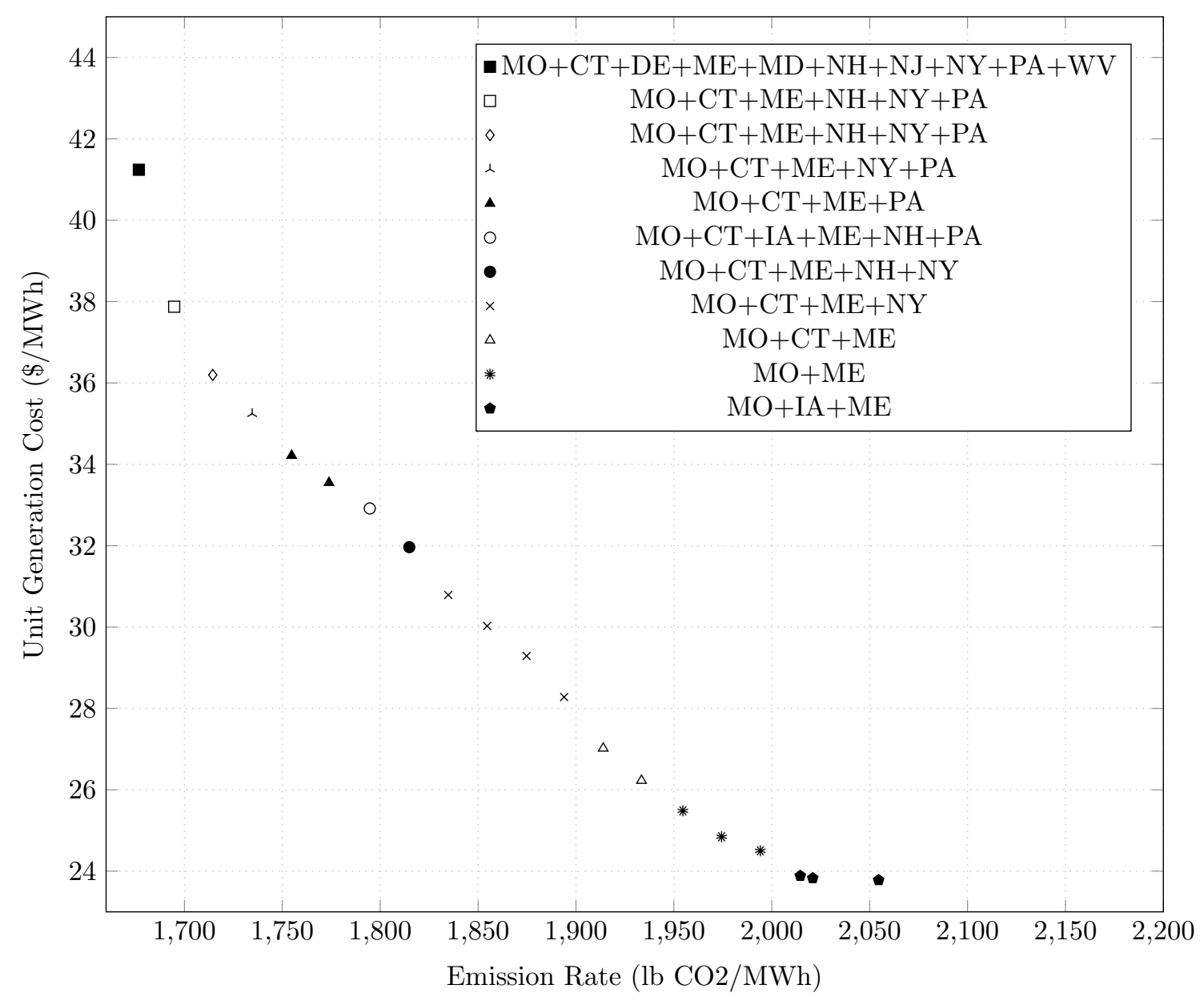

Figure 4.4: Efficient frontier of multi-state collaboration for $0 \%$ feasibility

Consider the model results corresponding to $\Psi=50 \%$ (Figure 4.5). Here, MO's efficient frontier is comprised of ten different multi-state collaborations. The minimum aggregate $\mathrm{CO}_{2}$ emission rate that can be achieved by $\mathrm{MO}$ through partnering is 1753 lb/MWh, with an electricity generation cost of $\$ 40.40 / \mathrm{MWh}$; this emission rate is achieved by partnering with NJ, NY, MD, PA, and WV. The minimum-achievable 


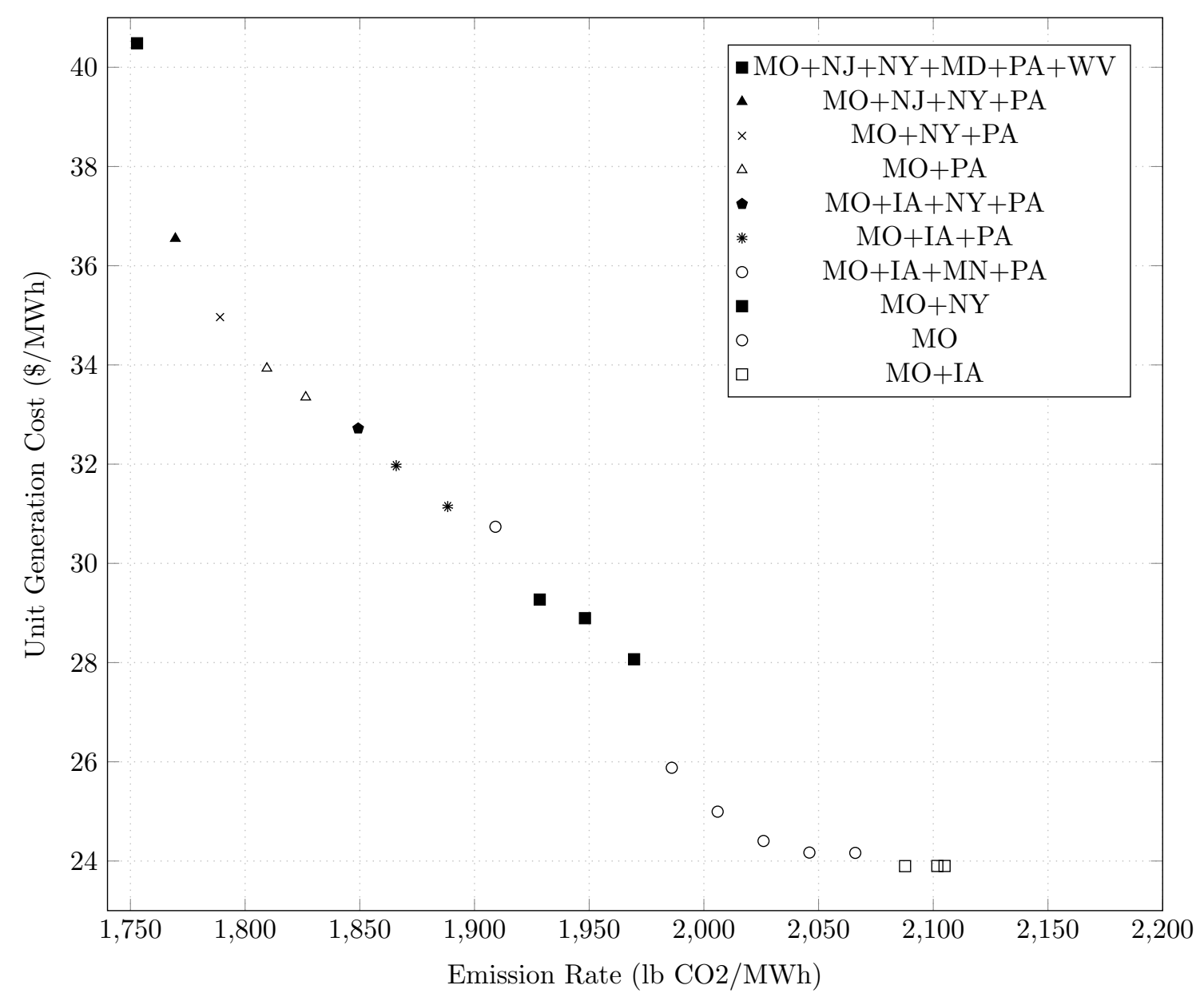

Figure 4.5: Efficient frontier of multi-state collaboration for $50 \%$ feasibility

aggregate cost of $\$ 23.90 / \mathrm{MWh}$ can be achieved by partnering with IA, this solution has aggregate emissions of $2102 \mathrm{lb} / \mathrm{MWh}$.

In separate model runs at $\Psi=50 \%$, we determined that MO's minimum-achievable emission level when operating alone is $1986 \mathrm{lb} / \mathrm{MWh}$ at electricity generation cost of $\$ 25.88 / \mathrm{MWh}$. This solution is on the efficient frontier presented in Figure 4.5; in fact, between emission rates of 1986 and $2066 \mathrm{lb} / \mathrm{MWh}$, the minimum cost solution has MO operating alone, with no partner states. This is primarily due to the fact that MO's electricity generation cost from coal is relatively low when compared to 


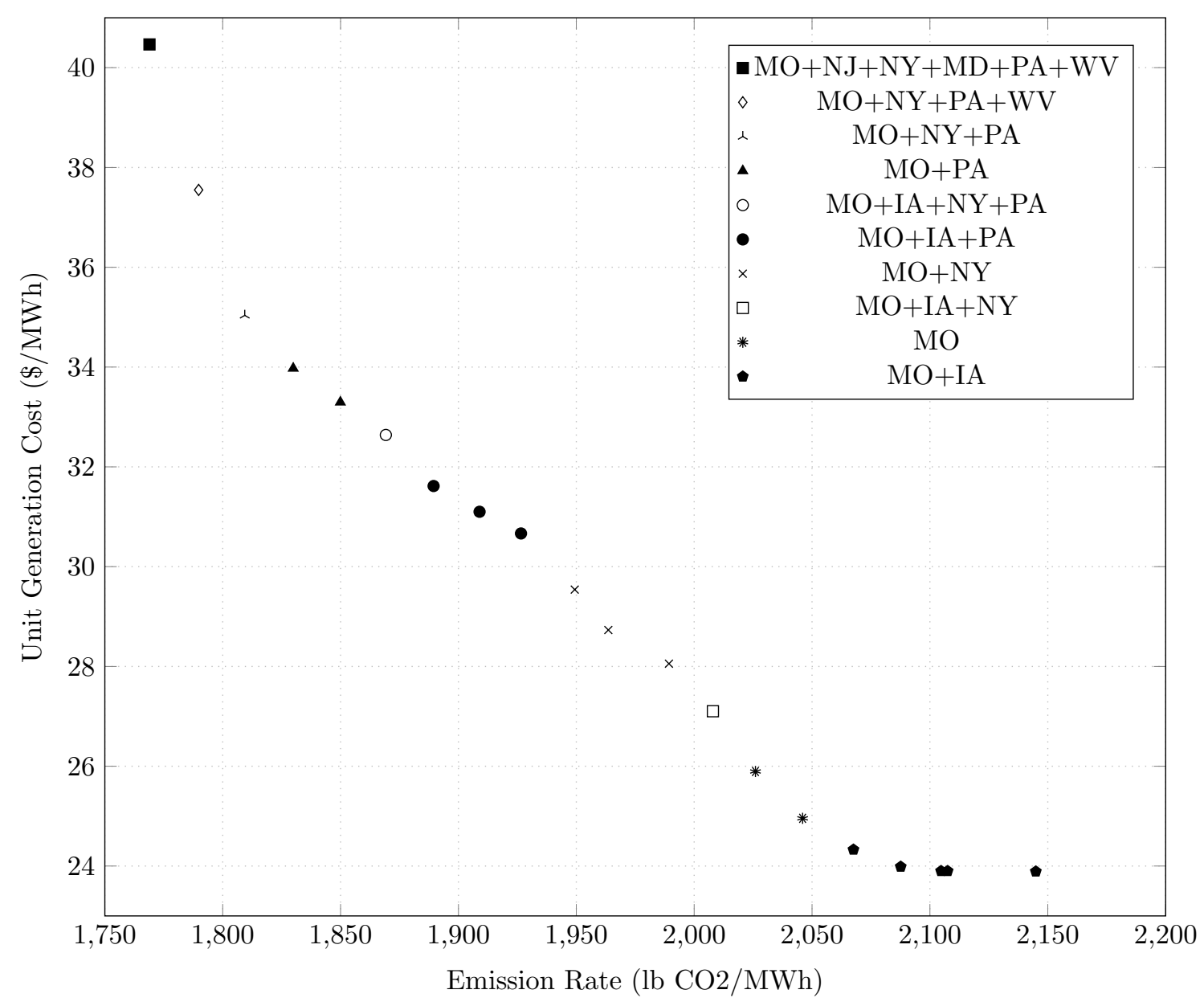

Figure 4.6: Efficient frontier of multi-state collaboration for $90 \%$ feasibility

the other candidate states, as shown in Table 4.1.

However, should MO wish to achieve an emissions level less than $1986 \mathrm{lb} / \mathrm{MWh}$, collaboration with other states is necessary. Suppose that MO wished to achieve an aggregate emissions rate of $1866 \mathrm{lb} / \mathrm{MWh}$ (a reduction of $6 \%$, compared to the minimum emission rate it can achieve by operating independently) at a minimum feasibility probability of $50 \%$. From Figure 4.5 we observe that this could be achieved at a minimum cost of $\$ 31.80 / \mathrm{MWh}$ were MO to enter into a partnership with IA and PA. However, these aggregate emissions and cost values achieved under this part- 


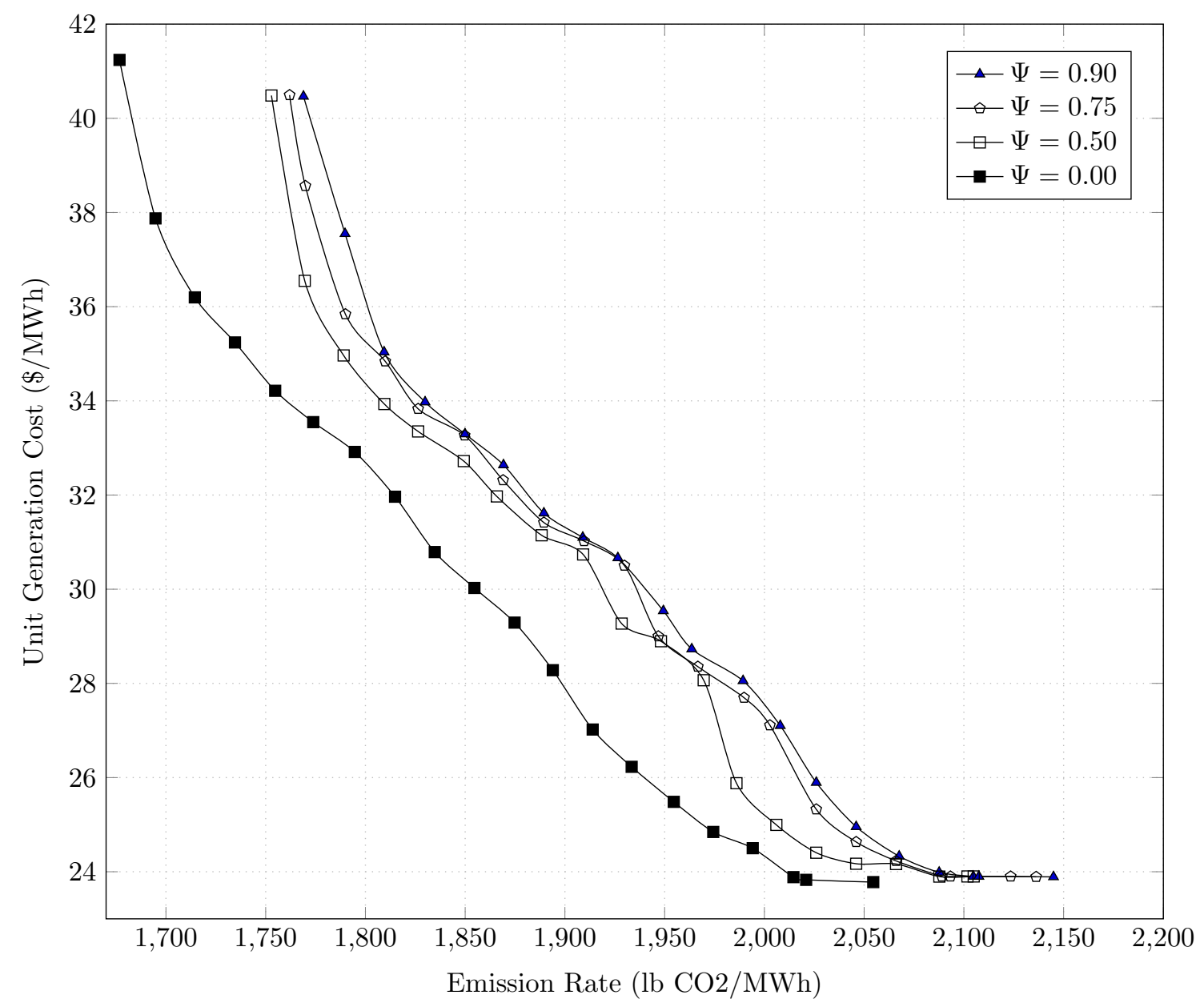

Figure 4.7: Efficient frontiers across different feasibility probabilities

nership mask some variability across the partners. Observe in Table 4.5 that PA is contributing most of the biomass generation in this partnership. Under the partnership, power plants in PA are exercising their relative advantage to utilize biomass to reduce the partnership's aggregate emissions. Were PA to operate independently, it could satisfy its own electricity generation requirements, at a similar emission level (1858 lb/MWh), at feasibility probability of $50 \%$, all at at cost of $\$ 37.54 / \mathrm{MWh}$. Given that the cost in PA under the partnership has increased to $\$ 39.55$, to achieve 
Table 4.5: Contributions among partners in one multi-state collaboration

\begin{tabular}{|c|cc|c|c|c|}
\hline State & \multicolumn{2}{|c|}{$\begin{array}{c}\text { Annual generation } \\
\text { (millions of MWh) from: }\end{array}$} & $\begin{array}{c}\text { Emission rate } \\
\text { (lb } \mathbf{C O}_{2} / \mathbf{M W h} \text { ) } \\
\text { biomass utilized }\end{array}$ & Prob. of Feasibility & \$/MWh \\
\hline MO & 50.6 & 2.7 & 2,026 & 91 & 25.01 \\
IA & 33.0 & 2.1 & 2,060 & 85 & 23.80 \\
PA & 67.3 & 14.4 & 1,678 & 71 & 39.55 \\
\hline
\end{tabular}

a comparable aggregate emissions level, some sort of arrangement would be needed by which the other partnering states (MO and IA, in this case) compensate PA, such that the overall cost efficiencies are shared across all partners. One simple notional scheme would be to allow for transfer payments from MO and IA to PA such that each state's net cost per MWh was equal to the partnership's aggregate cost rate (in this case, those payments would equal $27 \%$ and $33 \%$ of the total generation cost in MO and IA, respectively).

As demonstrated in Figure 4.7, the electricity generation cost per MWh increases, at a fixed emissions level, when the probability of feasibility increases. For example, at $\mathrm{CO}_{2}$ emission rate of $1774 \mathrm{lb} / \mathrm{MWh}$, the aggregate cost is $\$ 33.55 / \mathrm{MWh}$ for $\Psi=0 \%$ and $\$ 36.20 / \mathrm{MWh}$ for $\Psi=50 \%$; this increase of $7.9 \%$ can be considered the "price of robustness" (at $\Psi=75 \%$ and $\Psi=90 \%$, these corresponding cost increases are $13.4 \%$ and $18.6 \%$, respectively). Moreover, the minimum-achievable emission rate increases as the probability of feasibility increases, from $1677 \mathrm{lb} / \mathrm{MWh}$ at $\Psi=0 \%$ to 1769 $\mathrm{lb} / \mathrm{MWh}$ at $\Psi=90 \%$. 


\section{Chapter 5}

\section{A Demand Response Model for Biopower Generation to Identify Optimal Multi-State Collaborations}

In this chapter, we will extend this study by incorporating the price elasticity of demand. An econometric model is deployed to investigate the impact of energy policy-related regulations on woody biomass price paid by power plants. We then incorporated that model into our previously developed robust optimization model to identify min-cost approaches for reducing carbon emissions via biomass co-firing subject to spatially-explicit biomass availability constraints. A two-stage approach is utilized to solve the resultant robust mixed integer nonlinear programming (MINLP) model. We apply this model to a set of 18 states in the northern US to identify optimal sets of multi-state collaborations, subject to a constraint on the probability satisfying the desired emission targets. 


\subsection{Demand Response Model Formulation}

In this chapter, a demand response model (DRM) is developed and incorporated in the MILP model that was discussed in chapter 4.1. In the following we will discuss how the DRM parameters estimated as well as the process of incorporating DRM in MILP model, which results in an MINLP.

\subsubsection{Estimation of the DRM parameters}

A constant elasticity demand response model is employed to estimate how the amount of woody biomass consumed impacts the woody biomass price. The constant elasticity model is represented as in following equation (5.1).

$$
P\left(v_{p r}^{t}\right)=\alpha_{p r}^{t}\left(v_{p r}^{t}\right)^{\beta}
$$

Here $v_{p r}^{t}$ is the amount of woody biomass consumed from power plant $p$ within radius $r$ at time $t$. $\beta$ is the demand elasticity of price, and $\alpha_{p r}^{t}$ is a unique value for each

power plant $p$ and radius $r . P\left(v_{p r}^{t}\right)$ is the price of woody biomass depending on $v_{p r}^{t}$; therefore, the price of woody biomass becomes a decision variable. As Huang et al. (2013) mentioned, the estimation of the parameter of the constant elasticity model can easily be determined by taking the logarithm of the constant-elasticity model to transform it into a linear function. Therefore, in order to estimate the coefficient of the elasticity model, we take the natural log of both sides, and the equation becomes as follows.

$$
\ln \left(P\left(v_{p r}^{t}\right)\right)=\ln \left(\alpha_{p r}^{t}\right)+\beta \ln \left(v_{p r}^{t}\right)
$$


Here, $\ln \left(\alpha_{p r}^{t}\right)$ and $\beta$ are parameters that can be estimated by least-squares regression. We used log-transformation to estimate the transformed relationship between the data of woody biomass consumption and woody biomass price as shown in Figure 5.1. Data utilized to estimate the parameters in Figure 5.1 is based on historical data from Energy Information Administration(EIA) data covering the interval between 2001 and 2014. The data for wood energy consumed by the electric power sector was provided from EIA (2017b) database. Woody biomass price data was retrieved from another EIA (2014b) database. Based on these log-transformed data, the estimated $\beta$ value is 1.3075 across this national-level data set. We assume that this same elasticity value applies across all power plants included in this study. That means that a one percent increase in the demand is associated with a 1.3075 percent raise in the woody biomass price. Based on the regression presented in Figure 5.1, the adjusted R-square $\left(R^{2}\right)$ value is 0.61 , indicating reasonably good fit.

A common $\alpha$ value for all power plants did not work well with power plant specific woody prices, consisted with the discussion in Huang et al. (2013). That is why we need to determine a plant-specific $\alpha$ for each power plant. In order to estimate a plant-specific $\alpha_{p 1}^{t}$ value for each plant's initial $30 \mathrm{~km}$ radius, it is assumed that the price function starts at some positive $K_{p 1}^{t}$ value. $K_{p 1}^{t}$ value was estimated based on the following equation.

$$
K_{p 1}^{t}=\bar{\zeta}_{p 1}^{t}-\left(\frac{\bar{\zeta}_{p 2}^{t}-\bar{\zeta}_{p 1}^{t}}{2}\right)
$$

$\bar{\zeta}_{p 1}^{t}$ is the average price of woody biomass per ton for plant $p$ for biomass procured within a $30 \mathrm{~km}$ radius at time period $t$, estimated previously by Goerndt et al. (2013). $\bar{\zeta}_{p 2}^{t}$ is the similar average price of woody biomass per ton for plant $p$ within a $60 \mathrm{~km}$ 


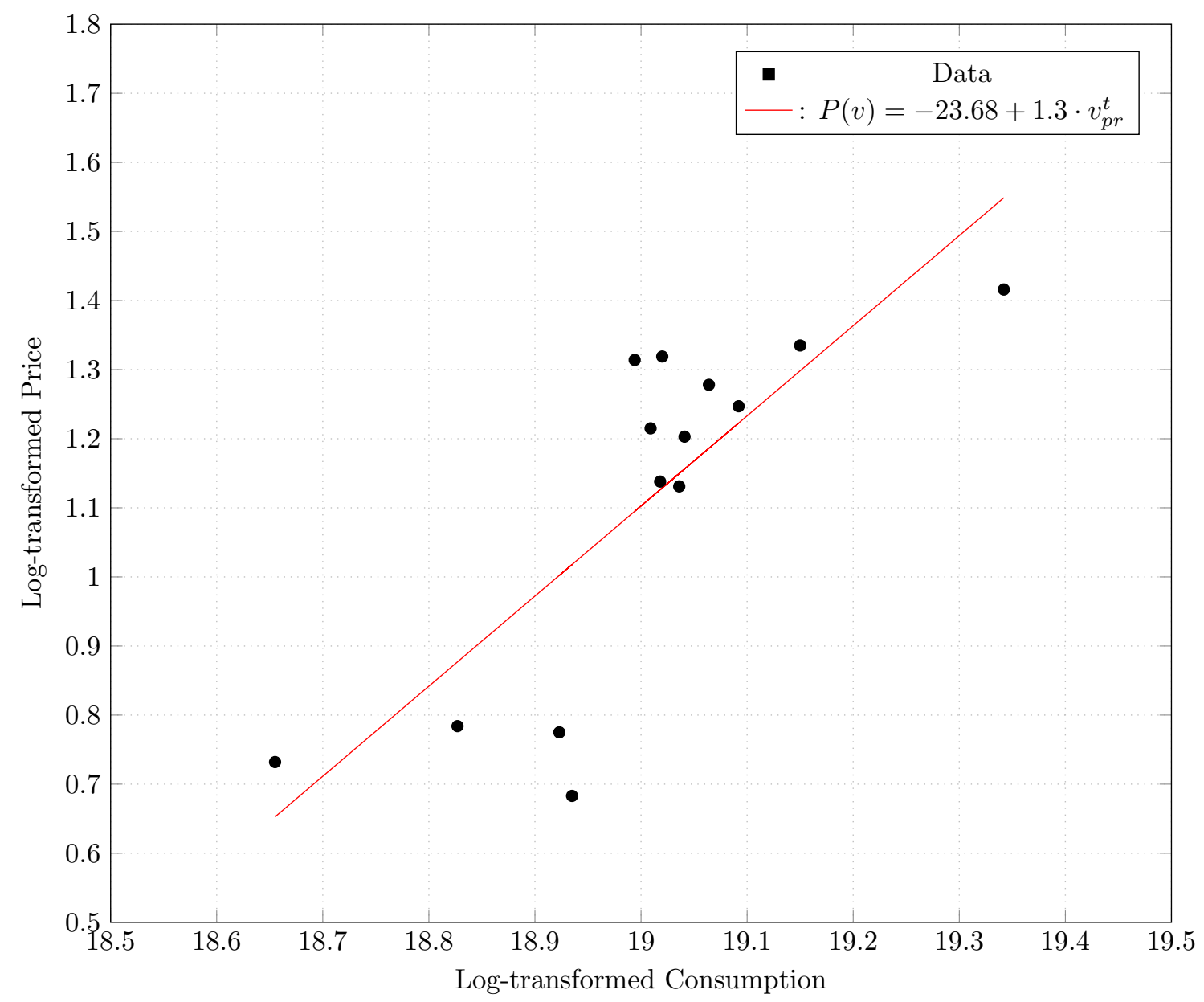

Figure 5.1: Log-transformed woody biomass consumption and its price

radius at time period $t$.

Recall that, $\varphi_{r}$ is the potential available woody biomass of each power plant within $30 \mathrm{~km}, 60 \mathrm{~km}$, and $90 \mathrm{~km}$ radius were already obtained. The following Equation 5.4 is used to estimate the $\alpha_{p 1}^{t}$ value of the demand response function within $30 \mathrm{~km}$. The left side of equation is the area $A_{1}$ under the price function $K_{p 1}^{t}+\alpha_{p 1}^{t} v_{p 1}^{t}$ as shown in Figure 5.2. The right hand side of equation is $\bar{\zeta}_{p 1}^{t} \varphi_{1}$, which is the total cost if $\varphi_{1}$ tons are consumed at the average price $\bar{\zeta}_{p 1}^{t}$. 


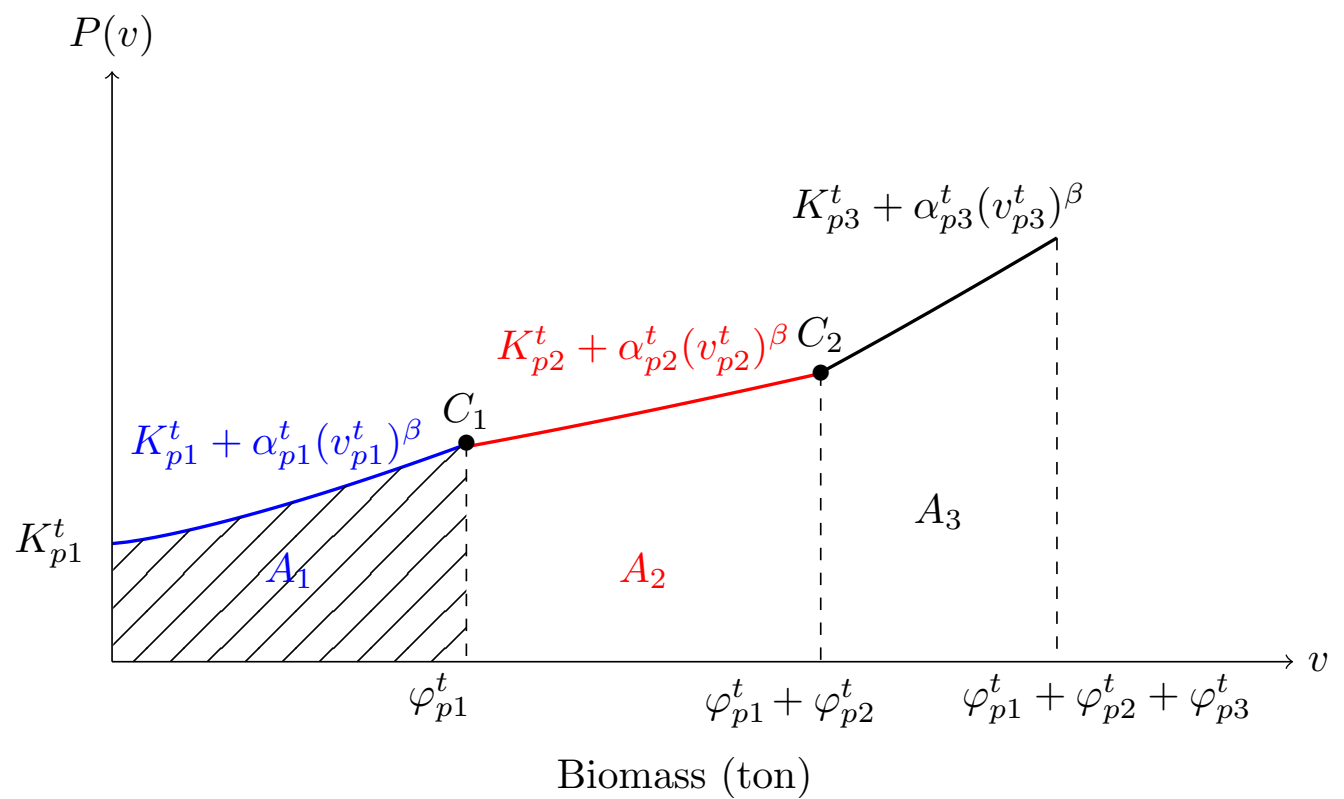

Figure 5.2: Demand response function $\mathrm{P}(\mathrm{v})$ for different radius

$$
\int_{0}^{\varphi_{1}} K_{p 1}^{t}+\alpha_{p 1}^{t}\left(v_{p 1}^{t}\right)^{\beta} d v=\bar{\zeta}_{p 1}^{t} \varphi_{1}
$$

In order to estimate $\alpha_{p r}^{t}$ and $K_{p r}^{t}$ and values for radii $60 \mathrm{~km}$ and $90 \mathrm{~km}$, we assumed that the demand response function is continuous across each radius breakpoint $\varphi_{r}$. As shown in Figure 5.2, the point $C_{1}$ is connected between the price function for $30 \mathrm{~km}$ and $60 \mathrm{~km}$. The $K_{p 2}^{t}$ and $\alpha_{p 2}^{t}$ are the unknown variables in equation 5.5 .

$$
K_{p 2}^{t}+\alpha_{p 2}^{t}\left(\varphi_{1}\right)^{\beta}=K_{p 1}^{t}+\alpha_{p 1}^{t}\left(\varphi_{1}\right)^{\beta}
$$

It is also assumed that as seen in Figure 5.2, the average biomass consumption cost $A_{2}$, the area under the price function $K_{p 2}^{t}+\alpha_{p 2}^{t}\left(v_{p 2}^{t}\right)^{\beta}$, equals to the total cost 
to obtain all tons between $\varphi_{2}$ and $\varphi_{1}$. The corresponding formulation for the second assumption is given in equation 5.6. In this equation, $K_{p 2}^{t}$ and $\alpha_{p 2}^{t}$ are also unknown variables. Now, we have a system with two equations (5.5 and 5.6) and with two unknown variables $K_{p 2}^{t}$ and $\alpha_{p 2}^{t}$. From that, it is straightforward to derive $K_{p 2}^{t}$ and $\alpha_{p 2}^{t}$ values.

$$
\int_{\varphi_{1}}^{\varphi_{1}+\varphi_{2}} K_{p 2}^{t}+\alpha_{p 2}^{t}\left(v_{p 2}^{t}\right)^{\beta} d v=\bar{\zeta}_{p 2}^{t}\left(\varphi_{2}\right)
$$

The same steps were followed in computing the $K_{p 3}^{t}$ and $\alpha_{p 3}^{t}$ for each power plant.

\subsubsection{The mixed integer nonlinear objective function}

The objective function including the demand respond model formulation is identified in the following term.

$$
\begin{aligned}
\min \sum_{t \in \mathcal{T}} \sum_{p \in \mathcal{P}} \sigma_{p}^{t} x_{p}^{t}+\sum_{t \in \mathcal{T}} \sum_{p \in \mathcal{P}} \gamma_{p}^{t} w_{p}^{t}+\sum_{t \in \mathcal{T}} \sum_{p \in \mathcal{P}} \sum_{r \in \mathcal{R}} P\left(v_{p r}^{t}\right) v_{p r}^{t}+\sum_{t \in \mathcal{T}} \sum_{p \in \mathcal{P}} \lambda_{p}^{t} y_{p}^{t}+\sum_{t \in \mathcal{T}} \sum_{p \in \mathcal{P}} \rho_{p}^{t} z_{p}^{t} \\
+\bar{z}_{0} \Gamma_{0}+\sum_{(t, p) \in \mathcal{P}_{0}} \tilde{\omega}_{t p}^{0}+\sum_{(t, p) \in \mathcal{P}_{1}} \bar{\omega}_{t p}^{0}
\end{aligned}
$$

In above objective function $5.7, P\left(v_{p r}^{t}\right)$ is a price function that shows the relationship between the the amount of woody biomass consumed and the woody biomass price. $v_{p r}^{t}$ is the amount of woody biomass used in the cofiring. $\sum_{t \in \mathcal{T}} \sum_{p \in \mathcal{P}} \sum_{r \in \mathcal{R}} P\left(v_{p r}^{t}\right) v_{p r}^{t}$ calculates the procurement cost of woody biomass cofired with coal. Notice that the term $P\left(v_{p r}^{t}\right) v_{p r}^{t}$ contains a multiplication of two variables. This term makes objective function nonlinear. 
The term $\sum_{t \in \mathcal{T}} \sum_{p \in \mathcal{P}} \sum_{r \in \mathcal{R}} P\left(v_{p r}^{t}\right) v_{p r}^{t}$ in the objective function that represents total procurement cost of woody biomass used in cofiring can be reformulated as given in the following equation 5.8, which is derived from the function exemplified in Figure 5.2.

$$
\begin{aligned}
\int_{0}^{\varphi_{1}}\left(K_{p 1}^{t}+\alpha_{p 1}^{t}\left(v_{p 1}^{t}\right)^{\beta}\right) d v+\int_{\varphi_{1}}^{\varphi_{1}+\varphi_{2}}\left(K_{p 2}^{t}\right. & \left.+\alpha_{p 2}^{t}\left(v_{p 2}^{t}\right)^{\beta}\right) d v \\
& +\int_{\varphi_{1}+\varphi_{2}}^{\varphi_{1}+\varphi_{2}+\varphi_{3}} K_{p 3}^{t}+\alpha_{p 3}^{t}\left(v_{p 3}^{t}\right)^{\beta} d v
\end{aligned}
$$

Equation (5.8) replaces the term $\sum_{t \in \mathcal{T}} \sum_{p \in \mathcal{P}} \sum_{r \in \mathcal{R}} P\left(v_{p r}^{t}\right) v_{p r}^{t}$. and the objective function becomes as given in term (5.9).

$$
\begin{aligned}
\min \sum_{t \in \mathcal{T}} \sum_{p \in \mathcal{P}} \sigma_{p}^{t} x_{p}^{t}+\sum_{t \in \mathcal{T}} \sum_{p \in \mathcal{P}} \gamma_{p}^{t} w_{p}^{t}+\sum_{t \in \mathcal{T}} \sum_{p \in \mathcal{P}} \int_{0}^{\varphi_{1}}\left(K_{p 1}^{t}+\alpha_{p 1}^{t}\left(v_{p 1}^{t}\right)^{\beta}\right) d v+ \\
\sum_{t \in \mathcal{T}} \sum_{p \in \mathcal{P}} \int_{\varphi_{1}}^{\varphi_{1}+\varphi_{2}}\left(K_{p 2}^{t}+\alpha_{p 2}^{t}\left(v_{p 2}^{t}\right)^{\beta}\right) d v+\sum_{t \in \mathcal{T}} \sum_{p \in \mathcal{P}} \int_{\varphi_{1}+\varphi_{2}}^{\varphi_{1}+\varphi_{2}+\varphi_{3}} K_{p 3}^{t}+\alpha_{p 3}^{t}\left(v_{p 3}^{t}\right)^{\beta} d v+ \\
\sum_{t \in \mathcal{T}} \sum_{p \in \mathcal{P}} \lambda_{p}^{t} y_{p}^{t}+\sum_{t \in \mathcal{T}} \sum_{p \in \mathcal{P}} \rho_{p}^{t} z_{p}^{t}+\bar{z}_{0} \Gamma_{0}+\sum_{(t, p) \in \mathcal{P}_{0}} \tilde{\omega}_{t p}^{0}+\sum_{(t, p) \in \mathcal{P}_{1}} \bar{\omega}_{t p}^{0}
\end{aligned}
$$

If we take the integration of the terms in the objective function, the ultimate formu- 
lation of the objective function becomes as follows.

$$
\begin{aligned}
& \min \sum_{t \in \mathcal{T}} \sum_{p \in \mathcal{P}} \sigma_{p}^{t} x_{p}^{t}+\sum_{t \in \mathcal{T}} \sum_{p \in \mathcal{P}} \gamma_{p}^{t} w_{p}^{t}+\sum_{t \in \mathcal{T}} \sum_{p \in \mathcal{P}} \sum_{r \in \mathcal{R}} K_{p r}^{t} v_{p r}^{t}+ \\
& \sum_{t \in \mathcal{T}} \sum_{p \in \mathcal{P}} \alpha_{p 1}^{t} \frac{\left(v_{p 1}^{t}\right)^{\beta+1}}{\beta+1}+\sum_{t \in \mathcal{T}} \sum_{p \in \mathcal{P}} \alpha_{p 2}^{t}\left(\frac{\left(\varphi_{p 1}^{t}+v_{p 2}^{t}\right)^{\beta+1}}{\beta+1}-\frac{\left(\varphi_{p 1}^{t}\right)^{\beta+1}}{\beta+1}\right)+ \\
& \sum_{t \in \mathcal{T}} \sum_{p \in \mathcal{P}} \alpha_{p 3}^{t}\left(\frac{\left(\varphi_{p 1}^{t}+\varphi_{p 2}^{t}+v_{p 3}^{t}\right)^{\beta+1}}{\beta+1}-\frac{\left(\varphi_{p 1}^{t}+\varphi_{p 2}^{t}\right)^{\beta+1}}{\beta+1}\right)+\sum_{t \in \mathcal{T}} \sum_{p \in \mathcal{P}} \lambda_{p}^{t} y_{p}^{t}+ \\
& \sum_{t \in \mathcal{T}} \sum_{p \in \mathcal{P}} \rho_{p}^{t} z_{p}^{t}+\bar{z}_{0} \Gamma_{0}+\sum_{(t, p) \in \mathcal{P}_{0}} \tilde{\omega}_{t p}^{0}+\sum_{(t, p) \in \mathcal{P}_{1}} \bar{\omega}_{t p}^{0}
\end{aligned}
$$

Given our previous estimates of the elasticity term $\beta=1.3075$, the coefficient of the $v_{p r}^{t}$ terms in objective function (5.10) is 2.3075, resulting in a nonlinear objective function.

\subsubsection{Demand response model constraints}

Following constraint (5.11) is added along with the demand response model, such that the amount of woody biomass that can be cofired cannot be more than maximum available woody biomass within radius $r$ for each power plant $p$ during time period $t$

$$
v_{p r}^{t} \leq \varphi_{p r}^{t}, \quad \forall t, p, r
$$

The constraints used with objective function (5.10) in our new MINLP were described in detail in previous chapters, thus here we present only their only mathematical formulations. 


$$
\begin{aligned}
& v_{p}^{t}=\frac{y_{p}^{t}}{\xi}, \forall t \in \mathcal{T}, \quad \forall p \in \mathcal{P} \\
& \sum_{p \in \mathcal{P}} \alpha_{p s}\left(y_{p}^{t}+z_{p}^{t}\right) \geq \beta_{s}^{t}+\chi_{s}^{t}, \quad \forall t \in \mathcal{T}, \quad \forall s \in \mathcal{S} \\
& y_{p}^{t}+z_{p}^{t} \leq \psi_{p}, \quad \forall t \in \mathcal{T}, \quad \forall p \in \mathcal{P} \\
& \sum_{p \in \mathcal{P}} \alpha_{p s} y_{p}^{t} \geq \chi_{s}^{t}, \quad \forall t \in \mathcal{T}, \quad \forall s \in \mathcal{S}
\end{aligned}
$$

$$
\begin{aligned}
\sum_{t \in \mathcal{T}} x_{p}^{t} & \leq 1, \quad \forall p \in \mathcal{P} \\
\sum_{t \in \mathcal{T}} w_{p}^{t} & \leq 1, \quad \forall p \in \mathcal{P} \\
w_{p}^{t} & \leq \sum_{t^{\prime} \leq t} x_{p}^{t^{\prime}}, \quad \forall t \in \mathcal{T}, \quad \forall p \in \mathcal{P} \\
y_{p}^{t} & =a_{p}^{t}+b_{p}^{t}, \quad \forall t \in \mathcal{T}, \quad \forall p \in \mathcal{P} \\
a_{p}^{t} & \leq M\left(\sum_{t^{\prime} \leq t} x_{p}^{t^{\prime}}\right), \quad \forall t \in \mathcal{T}, \quad \forall p \in \mathcal{P} \\
a_{p}^{t} & \leq M\left(1-\sum_{t^{\prime} \leq t} w_{p}^{t^{\prime}}\right), \quad \forall t \in \mathcal{T}, \quad \forall p \in \mathcal{P} \\
b_{p}^{t} & \leq M\left(\sum_{t^{\prime} \leq t} w_{p}^{t^{\prime}}\right), \quad \forall t \in \mathcal{T}, \quad \forall p \in \mathcal{P}
\end{aligned}
$$




$$
\begin{aligned}
& c_{p}^{t} \leq M\left(1-\sum_{t^{\prime} \leq t} x_{p}^{t^{\prime}}\right), \quad \forall t \in \mathcal{T}, \quad \forall p \in \mathcal{P} \\
& d_{p}^{t} \leq M\left(\sum_{t^{\prime} \leq t} w_{p}^{t^{\prime}}\right), \quad \forall t \in \mathcal{T}, \quad \forall p \in \mathcal{P} \\
& e_{p}^{t} \leq M\left(\sum_{t^{\prime} \leq t} x_{p}^{t^{\prime}}\right), \quad \forall t \in \mathcal{T}, \quad \forall p \in \mathcal{P} \\
& e_{p}^{t} \leq M\left(1-\sum_{t^{\prime} \leq t} w_{p}^{t^{\prime}}\right), \quad \forall t \in \mathcal{T}, \quad \forall p \in \mathcal{P} \\
& f_{p}^{t} \leq M\left(1-\sum_{t^{\prime} \leq t} x_{p}^{t^{\prime}}\right), \quad \forall t \in \mathcal{T}, \quad \forall p \in \mathcal{P} \\
& \epsilon \leq \frac{a_{p}^{t}+c_{p}^{t}+d_{p}^{t}}{a_{p}^{t}+c_{p}^{t}+d_{p}^{t}+z_{p}^{t}} \leq \zeta, \quad \forall t \in \mathcal{T}, \quad \forall p \in \mathcal{P} \\
& \mu_{s} \leq \frac{b_{p}^{t}+e_{p}^{t}+f_{p}^{t}}{b_{p}^{t}+e_{p}^{t}+f_{p}^{t}+z_{p}^{t}} \leq \eta_{s}, \quad \forall t \in \mathcal{T}, \quad \forall p \in \mathcal{P}
\end{aligned}
$$




$$
\begin{gathered}
\sum_{p \in \mathcal{P}} \alpha_{p s}\left(\delta_{p}^{t} z_{p}^{t}\right)+\bar{z}_{s}^{t} \Gamma_{s}^{t}+\sum_{p \in \mathcal{P}_{c}^{t}} \hat{\omega}_{p s}^{t} \leq \phi_{s}^{t} \sum_{p \in \mathcal{P}} \alpha_{p s}\left(z_{p}^{t}+y_{p}^{t}\right), \quad \forall t, s \\
\bar{z}_{0}+\tilde{\omega}_{t p}^{0} \geq \hat{\gamma}_{t p} \tilde{\varrho}_{t p}, \quad \forall(t, p) \in \mathcal{P}_{0} \\
\bar{z}_{0}+\bar{\omega}_{t p}^{0} \geq \hat{\rho}_{t p} \bar{\varrho}_{t p}, \quad \forall(t, p) \in \mathcal{P}_{1} \\
\bar{z}_{s}^{t}+\hat{\omega}_{p s}^{t} \geq \hat{\delta}_{p s}^{t} \hat{\varrho}_{p}^{t}, \quad \forall p \in \mathcal{P}_{c}^{t}, \quad \forall s \\
-\hat{\varrho}_{p}^{t} \leq z_{p}^{t} \leq \hat{\varrho}_{p}^{t}, \quad \forall t, p \\
-\tilde{\varrho}_{t p} \leq w_{p}^{t} \leq \tilde{\varrho}_{t p}, \quad \forall t, p \\
-\bar{\varrho}_{t p} \leq z_{p}^{t} \leq \bar{\varrho}_{t p}, \quad \forall t, p \\
\tilde{\omega}_{t p}^{0} \geq 0, \quad \forall(t, p) \in \mathcal{P}_{0} \\
\bar{\omega}_{t p}^{0} \geq 0, \quad \forall(t, p) \in \mathcal{P}_{1} \\
\hat{\omega}_{p s}^{t} \geq 0, \quad \forall p \in \mathcal{P}_{c}^{t}, \quad \forall s \\
\bar{z}_{0} \geq 0 \quad \\
\bar{\varrho}_{t p}, \hat{\varrho}_{p}^{t} \geq 0, \quad \forall t, p \\
\bar{z}_{s}^{t} \geq 0, \quad \forall t, p
\end{gathered}
$$

\subsection{A two-stage solution approach}

We again utilize an ROMCIA approach to identify the efficient frontier for one state (MO) that shows a range of aggregated emission rates that could be achieved along with the corresponding minimum unit electricity generation cost for different feasibility rates. Our ROMCIA approach was described in a detail way in section 4.3. 
In order to apply ROMCIA, we needed to determine a Robust Marginal Cost Table (ROMCT) for each state. To solve the single state MINLP necessary to populate each ROMCT, a two-stage approach was applied in GAMS. The framework this of two-stage approach is given in Figure (5.3). In the first stage, a quadratic approximation to nonlinear objective function (5.10) is used to obtain an initial solution for the values MILNP model. This approach works well in practice, since solving our quadratic mixed integer programming model with CPLEX takes relatively little computational time. This initial solution, which is necessarily feasible to the true MINLP, then allows the MINLP model to be solved in a reasonable time. Therefore, we first solve a model with constraints (5.10) -(5.42) and quadratic approximation objective function

$$
\begin{gathered}
\min \sum_{t \in \mathcal{T}} \sum_{p \in \mathcal{P}} \sigma_{p}^{t} x_{p}^{t}+\sum_{t \in \mathcal{T}} \sum_{p \in \mathcal{P}} \gamma_{p}^{t} w_{p}^{t}+\sum_{t \in \mathcal{T}} \sum_{p \in \mathcal{P}} \sum_{r \in \mathcal{R}} K_{p r}^{t} v_{p r}^{t}+\alpha_{p 1}^{t} \frac{\left(v_{p 1}^{t}\right)^{2}}{2}+ \\
\alpha_{p 2}^{t}\left(\frac{\left(\varphi_{p 1}^{t}+v_{p 2}^{t}\right)^{2}}{2}-\frac{\left(\varphi_{p 1}^{t}\right)^{2}}{2}\right)+\alpha_{p 3}^{t}\left(\frac{\left(\varphi_{p 1}^{t}+\varphi_{p 2}^{t}+v_{p 3}^{t}\right)^{2}}{2}-\frac{\left(\varphi_{p 1}^{t}+\varphi_{p 2}^{t}\right)^{2}}{2}\right)+ \\
\sum_{t \in \mathcal{T}} \sum_{p \in \mathcal{P}} \lambda_{p}^{t} y_{p}^{t}+\sum_{t \in \mathcal{T}} \sum_{p \in \mathcal{P}} \rho_{p}^{t} z_{p}^{t}+\bar{z}_{0} \Gamma_{0}+\sum_{(t, p) \in \mathcal{P}_{0}} \tilde{\omega}_{t p}^{0}+\sum_{(t, p) \in \mathcal{P}_{1}} \bar{\omega}_{t p}^{0}
\end{gathered}
$$

Observe that in this first stage, we are replacing the $1+\beta=2.3075$ objective coefficient for the $v_{p r}^{t}$ terms in objective (5.10) with a coefficient of 2 in objective (5.43). In the second stage, we used the ANTIGONE solver in GAMS to solve our true MINLP model. Misener \& Floudas (2014) tested ANTIGONE for various general MINLP, and found ANTIGONE to generally outperform other general purpose solvers. 


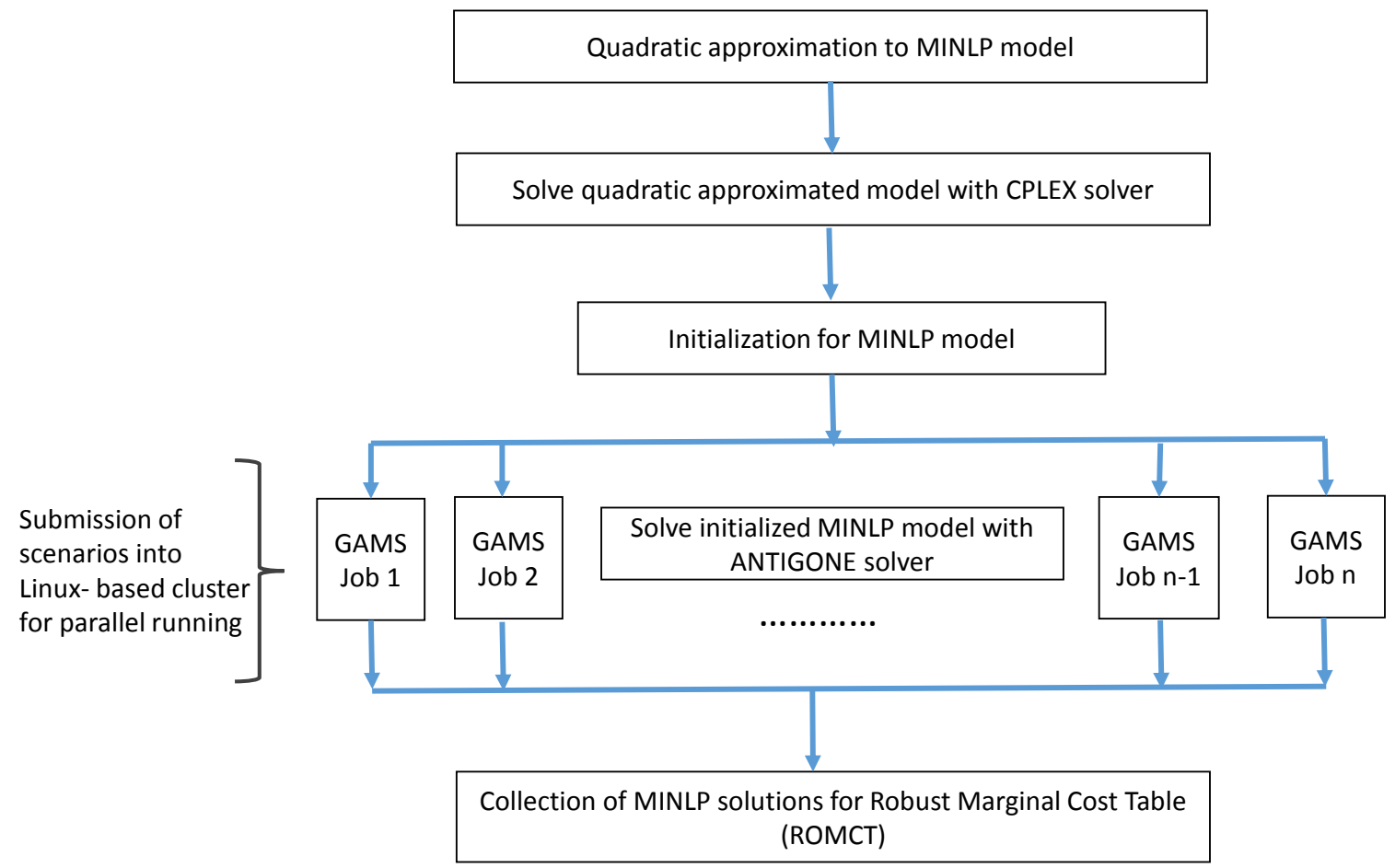

Figure 5.3: A two-stage approach to solve single state MINLP model

\subsubsection{Grid Computing}

The number of two-stage MINLP optimizations to be solved to create a state's ROMCT depends upon the state's electricity demand, range of potential emission levels, and number of power plants. The ranges of these potential values for the 18 states under consideration are provided in Table 5.1. For all states except PA, the increments between ROMCT table entries for demand level and emission rate are 1,000,000 MWh and $10 \mathrm{lb} / \mathrm{MWh}$, respectively. However, due to the large number of power plants in PA, we consider an increment demand level of 3,000,000 MWh and increment rate of $30 \mathrm{lb} / \mathrm{MWh}$ to generate scenarios in its ROMCT. The large number of power plants in PA both increases the number of model runs that must be 
Table 5.1: Demand levels, Emission Rates, and Gamma levels considered to create MCT for each state

\begin{tabular}{|l||l|l|l|l|l|l|l|l|}
\hline \multicolumn{1}{|c|}{} & \multicolumn{3}{l|}{ Demand Levels $(D)$} & \multicolumn{3}{l|}{ Emission Rates $(E)$} & \multicolumn{2}{l|}{ Gamma $(G)$} \\
\cline { 2 - 8 } & Start & End & Increment & Start & End & Increment & Start & End \\
\hline \hline CT & $1,000,000$ & $2,000,000$ & $1,000,000$ & 900 & 1890 & 10 & 0 & 2 \\
DE & $1,000,000$ & $2,000,000$ & $1,000,000$ & 1300 & 2000 & 10 & 0 & 2 \\
IA & $10,000,000$ & $36,000,000$ & $1,000,000$ & 1900 & 2160 & 10 & 0 & 23 \\
IL & $10,000,000$ & $71,000,000$ & $1,000,000$ & 1980 & 2250 & 10 & 0 & 21 \\
IN & $1,000,000$ & $17,000,000$ & $1,000,000$ & 2100 & 2360 & 10 & 0 & 4 \\
MA & $1,000,000$ & $8,000,000$ & $1,000,000$ & 1680 & 2000 & 10 & 0 & 5 \\
ME & 15,000 & 150,000 & $1,000,000$ & 0 & 300 & 10 & 0 & 1 \\
MD & $1,000,000$ & $23,000,000$ & $1,000,000$ & 1590 & 2200 & 10 & 0 & 9 \\
MI & $20,000,000$ & $60,000,000$ & $1,000,000$ & 1950 & 2200 & 10 & 0 & 21 \\
MN & $10,000,000$ & $26,000,000$ & $1,000,000$ & 1850 & 2250 & 10 & 0 & 13 \\
MO & $5,000,000$ & $60,000,000$ & $1,000,000$ & 1950 & 2210 & 10 & 0 & 14 \\
NH & $1,000,000$ & $2,000,000$ & $1,000,000$ & 1206 & 2330 & 10 & 0 & 1 \\
NJ & $1,000,000$ & $5,000,000$ & $1,000,000$ & 1090 & 2090 & 10 & 0 & 5 \\
NY & $1,000,000$ & $11,000,000$ & $1,000,000$ & 1475 & 2090 & 10 & 0 & 11 \\
OH & $25,000,000$ & $60,000,000$ & $1,000,000$ & 1754 & 2100 & 10 & 0 & 10 \\
PA & $20,000,000$ & $73,000,000$ & $3,000,000$ & 1620 & 2090 & 20 & 0 & 30 \\
WV & $22,000,000$ & $67,000,000$ & $1,000,000$ & 1660 & 2040 & 10 & 0 & 17 \\
WI & $10,000,000$ & $35,000,000$ & $1,000,000$ & 1950 & 2160 & 10 & 0 & 20 \\
\hline
\end{tabular}

performed (with 31 runs required for every combination of demand level and emission rate), and also makes it significantly harder to solve each individual MINLP model for due to the large number of decision variables. Note that, as indicated in the Table 5.1, Gamma levels range from 0 (corresponding to the non-robust case) to the number of the power plants in the state.

Were the two-stage MINLPs needed to populate an ROMCT solved on a single computer, the computational time would be excessive, given the very large number of runs to be performed (in excess of 10,000 for many states). Therefore, we utilized a grid computing framework in GAMS (2017), in a Linux-based cluster, to reduce the time necessary to create the ROMCTs. Bussieck et al. (2009) introduced a frame- 
work for modeling optimization problems in GAMS to utilize grid computing. We have utilized their framework to solve these problems using GAMS on the computing infrastructure provided by Research Computing Support Services of the University of Missouri. In the parallel computing environment, since the solutions of any two model runs used to populate an ROMCT don't depend on each other, these jobs can be submitted sequentially without waiting for the solution of the other run. The computational procedure used to generate the ROMCT for each state is given in $\mathrm{Al}-$ gorithm (1). In the first step of the procedure, the quadratic approximation (MIQCP) model is solved based on each considered combination of $d \in D, e \in E, g \in G$, and all variables' optimal solution is recorded to initialize the MINLP model. In step 1.3, the true MINLP model is generated and executed. In the step 1.4 of Algorithm (1) the model.handle function in GAMS handles each executed scenario $\mathrm{h}(\mathrm{d}, \mathrm{e}, \mathrm{g})$ without waiting the model being solved. In step 2 of Algorithm (1), having assigned all scenarios to grids for parallel running, Repeat loop collects all solved scenarios with function handleCollect $(h(d, e, g))$ Until all solutions are retrieved.

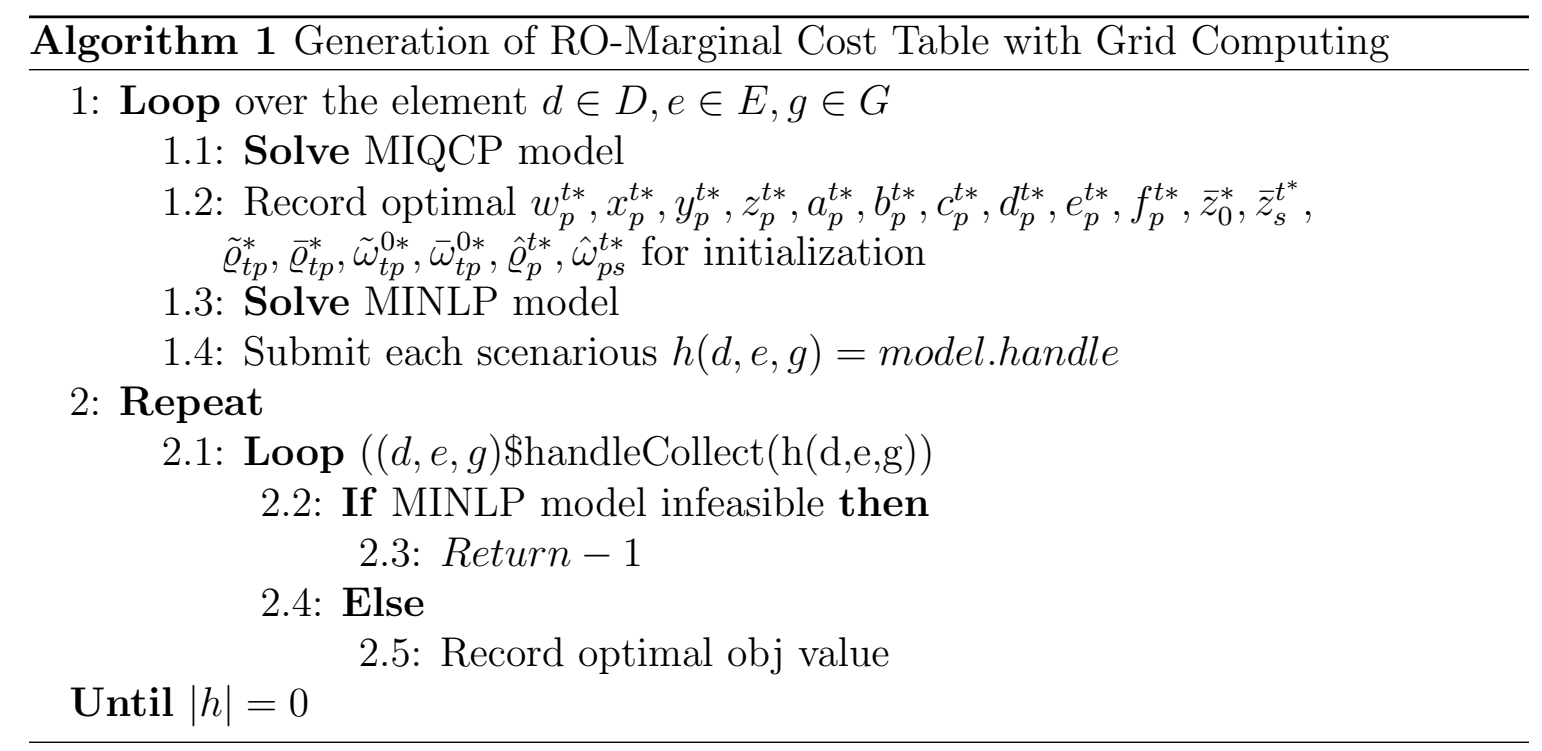


Table 5.2: The comparison of computation time of certain number of scenarios for PA with grid computing and MILP model

\begin{tabular}{|c|c|c|c|c|}
\hline Model & States & $\begin{array}{c}\text { Demand } \\
\text { (millions of MWh) }\end{array}$ & \# of Scenarios & $\begin{array}{c}\text { Running time } \\
\text { (min) }\end{array}$ \\
\hline MINLP & PA & 20 & 744 & 7,629 \\
MINLP Grid & PA & 20 & 744 & 629 \\
\hline
\end{tabular}

Grid computing significantly decreases the computational time by using parallel computing. As an example, we ran the model for a subset of runs (those with annual demand of 20,000,000 MWh) for PA, using both a serial procedure on a Windowsbased platform and on the linux-cluster with grid computing as given in Algorithm (1). As presented in Table (5.2), the grid computing in the linux-base cluster is more than 12 times faster than a serial-based approach in Windows. Table (5.3) presents the number of scenarios needed for each state's ROMCT, the optimality gap used by GAMS for the MINLP model, and the total running time needed to populate each state's ROMCT.

\subsubsection{Generation of the efficient frontiers for Multi-state plan}

As discussed in section 4.3, our ROMCIA model can be used to generate efficient frontier for a single state (such as MO). The efficient frontiers show the trade-off between electricity generation cost and emission rate under various robustness levels. The procedure that is used to generate the efficient frontiers is described in detail below. Define the following sets and notations to describe this procedure, as presented in Algorithm (2). $\mathcal{Q}$ is the set of emission rate scenarios considered to generate the efficient frontier, indexed by $q$. The step size that is used for the potential objective 
Table 5.3: Running times to generate single state' MCT

\begin{tabular}{|l||l|l|l||}
\hline & \# of Scenarios & Optimality Gap & Running Times(Min) \\
\hline \hline CT & 600 & 0.03 & 8 \\
DE & 426 & 0.03 & 6 \\
IA & 17,496 & 0.03 & 2,250 \\
IL & 38,192 & 0.05 & 29,359 \\
IN & 2,295 & 0.03 & 2,295 \\
MA & 1,584 & 0.03 & 1,584 \\
ME & 120 & 0.03 & 2 \\
MD & 14,260 & 0.03 & 4,893 \\
MI & 23,452 & 0.04 & 35,649 \\
MN & 9,758 & 0.05 & 1,158 \\
MO & 22,680 & 0.05 & 6,920 \\
NH & 454 & 0.03 & 8 \\
NJ & 3,030 & 0.03 & 88 \\
NY & 8,250 & 0.04 & 3,469 \\
OH & 14,098 & 0.05 & 8,872 \\
PA & 14,177 & 0.05 & 11,445 \\
WV & 32,292 & 0.03 & 41,005 \\
WI & 12,012 & 0.03 & 11,584 \\
\hline
\end{tabular}

function is denoted by $\ddot{s}$. $\tilde{E}$ is the minimum emission rate value to be considered. $\ddot{E}$ is the emission rate achieved the optimal MILP solution. $E_{q}$ is the emission rate value for scenario $q$ in the MILP optimal solution. $\bar{Z}_{q}$ is the optimal objective value for scenario $q$. $u_{q i j g s}$ is the electricity generation level $i$ is achieved at $\mathrm{CO}_{2}$ emission level $j$ for scenario $q$. $Z_{q}$ denotes the lower bound on objective function value.

As it can be seen from Algorithm (2), we ran GAMS/CPLEX multiple times, with different optimality gap of 0.05 and 0.001 to generate the efficient frontier. In step 1.0 from Algorithm (2), we loop over the set of emission rate scenarios $\mathcal{Q}$, emission rate scenarios, solving the MILP model described in section 4.3, with optimality gap 0.05. The step size, $\ddot{s}$, used in the first loop is 0.3 . With this step size, we are attempting to quickly obtain a good solution with a coarsely-defined linear approximation objective 
(5.43). In the second loop, step 2.0 from Algorithm (2) is used with a much smaller optimality gap 0.001. As described in step 2.3.3, a new step size, $\bar{s}$, is introduced to generate a new set of potential objective values. Here we search for a nearly-optimal solution using a much more precisely defined linear approximation objective (5.43). The exact calculation we utilize to generate the set of objective values is given in the step 2.3.4.1.

\subsection{Results and Discussions}

\subsubsection{Demand-price relationships at a single power plant}

To illustrate demand price relationship at a localized level, consider a single power plant in MO. For this, the demand for electricity generated from coal in MO was fixed to 50,000,000 MWh at emission rate $2200 \mathrm{lb} / \mathrm{MWh}$. Demand for biopower generation was solved across a range of values, starting from a minimum of $100 \mathrm{MWh}$ with an increment rate of $1000 \mathrm{MWh}$ for 100 scenarios. This set of runs was performed to show how biomass price is impacted by increased rates of biomass demand. As seen in Figure 5.4, the price of biomass behaves differently in each radius. At the lowest biopower demand level, $100 \mathrm{MWh}$, the woody biomass price is $\$ 36.75$ per ton, while it is $\$ 42.56$ per ton at the biopower demand level of 19, 100 MWh. Within the radius of $30 \mathrm{~km}$, the price varies according to the demand response model's parameters, following the function $K_{p 1}^{t}+\alpha_{p 1}^{t}\left(v_{p 1}^{t}\right)^{\beta}$. Within the $60 \mathrm{~km}$ radius, price

behaves based on the $K_{p 2}^{t}+\alpha_{p 2}^{t}\left(v_{p 2}^{t}\right)^{\beta}$, which corresponds to changes between $\$ 42.56$ /ton and \$46.32/ton based on woody biomass consumption as shown in Figure (5.4). 


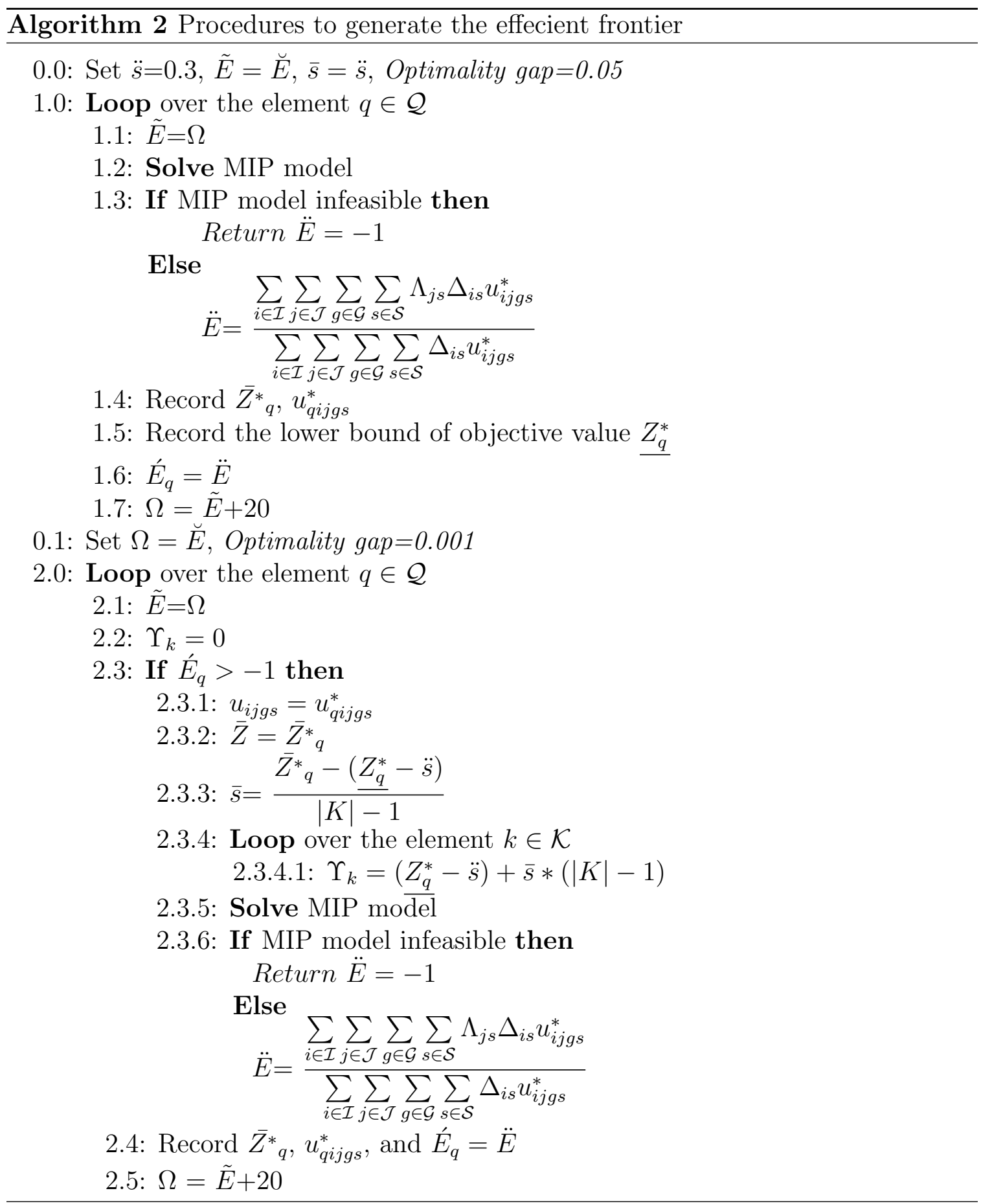




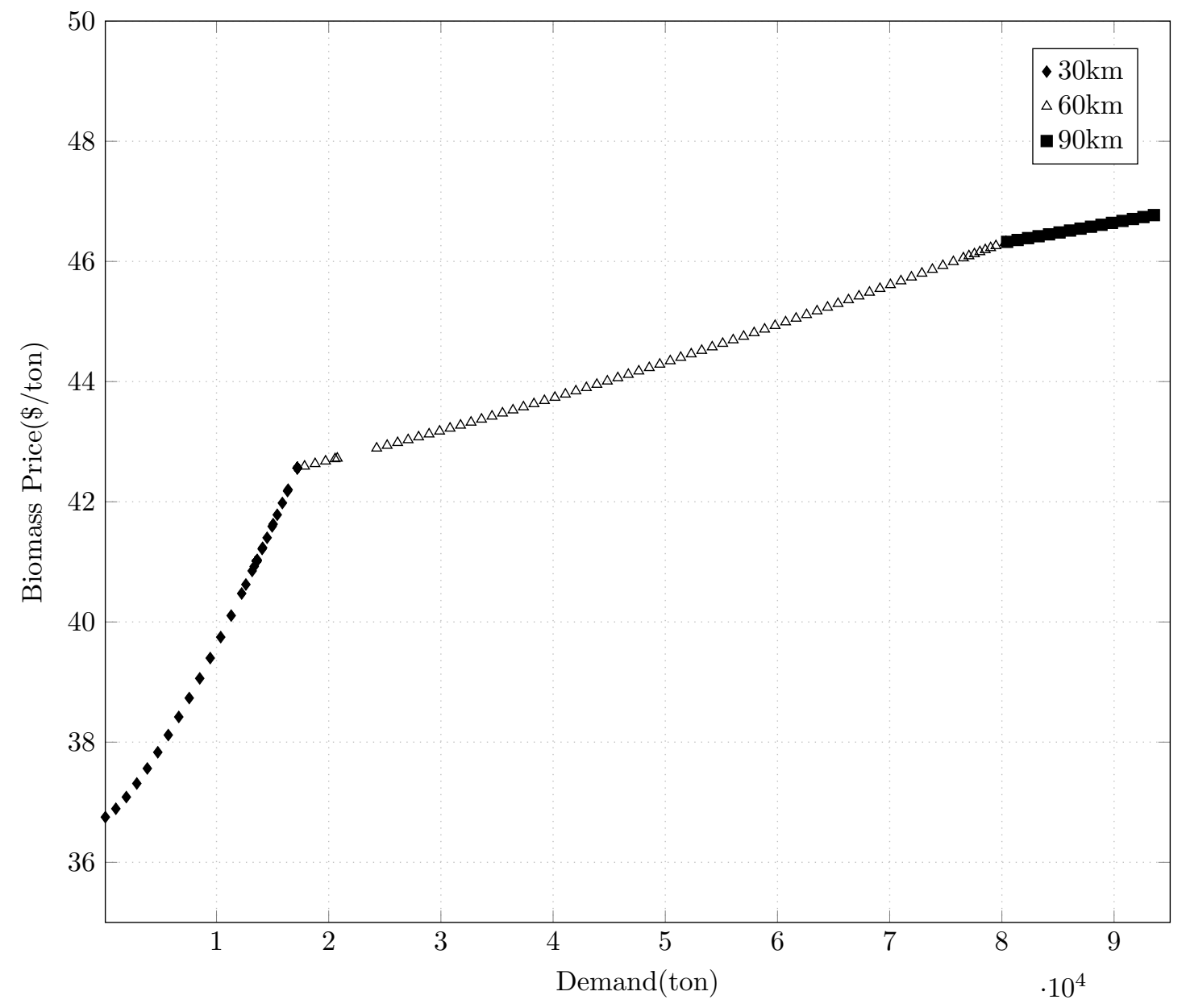

Figure 5.4: The impact of increased biomass demand on woody biomass price

\subsubsection{Multi-state efficient frontiers}

We ran the ROMCIA model to observe that how the efficient frontier of MO is affected by a range of emission rate and feasibility for $\Psi=0 \%, \Psi=50 \%$, and $\Psi=90 \%$. As seen in Figure (5.5), for feasibility $\Psi=0 \%$, (corresponding to a non-robust model), MO enters in 16 different multi-state partnerships between emission rates of 1662 $\mathrm{lb} / \mathrm{MWh}$ and $2030 \mathrm{lb} / \mathrm{MWh}$ with a corresponding unit electricity generation cost of $\$ 40.13 / \mathrm{MWh}$ and $\$ 22.42 / \mathrm{MWh}$. At that feasibility rate, the minimum achievable 


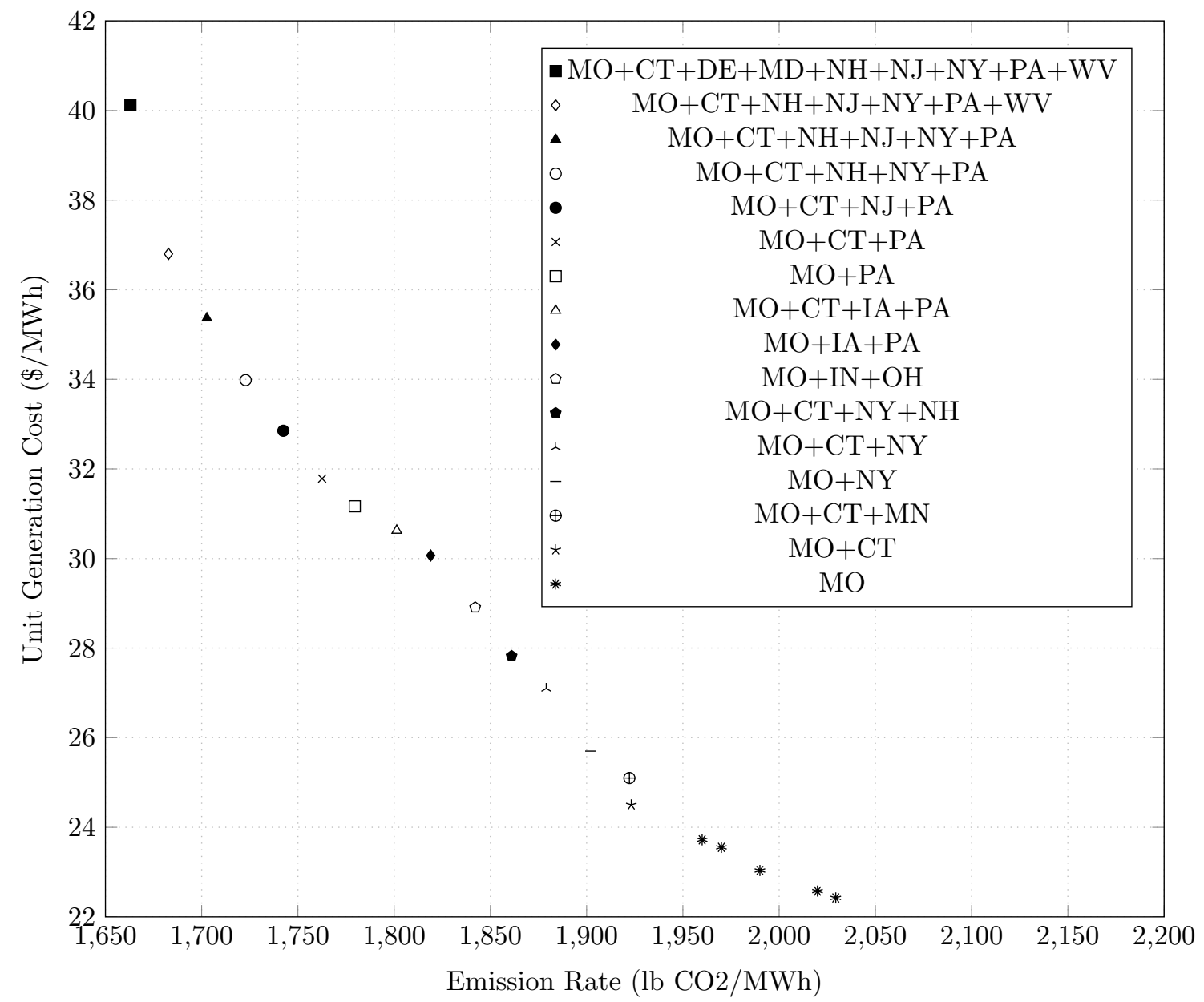

Figure 5.5: The efficient frontier of multi-state collaborations for nominal model,feasibility $\Psi=0 \%$

emission rate for MO is $1662 \mathrm{lb} / \mathrm{MWh}$, at which $\mathrm{MO}$ collaborates with CT, DE, MD, $\mathrm{NH}, \mathrm{NJ}, \mathrm{NY}, \mathrm{PA}$, and WV. Note that, within the emission range of $1960 \mathrm{lb} / \mathrm{MWh}$ and $2030 \mathrm{lb} / \mathrm{MWh}$, it is optimal for MO to act alone.

Based on the efficient frontier of feasibility $\Psi=0 \%$, the fluctuation in the aggregated woody biomass price is demonstrated in Figure (5.6). The aggregated price of woody biomass is significantly impacted by the emission rate. One reason is that MO collaborates with the states that have more potential woody biomass to reduce the 
emission rate, and the price of biomass in those states is higher than MO. The second reason is that more woody biomass is required to achieve a lower emission rate. This increases the demand for biomass, hence the price of woody biomass rises.

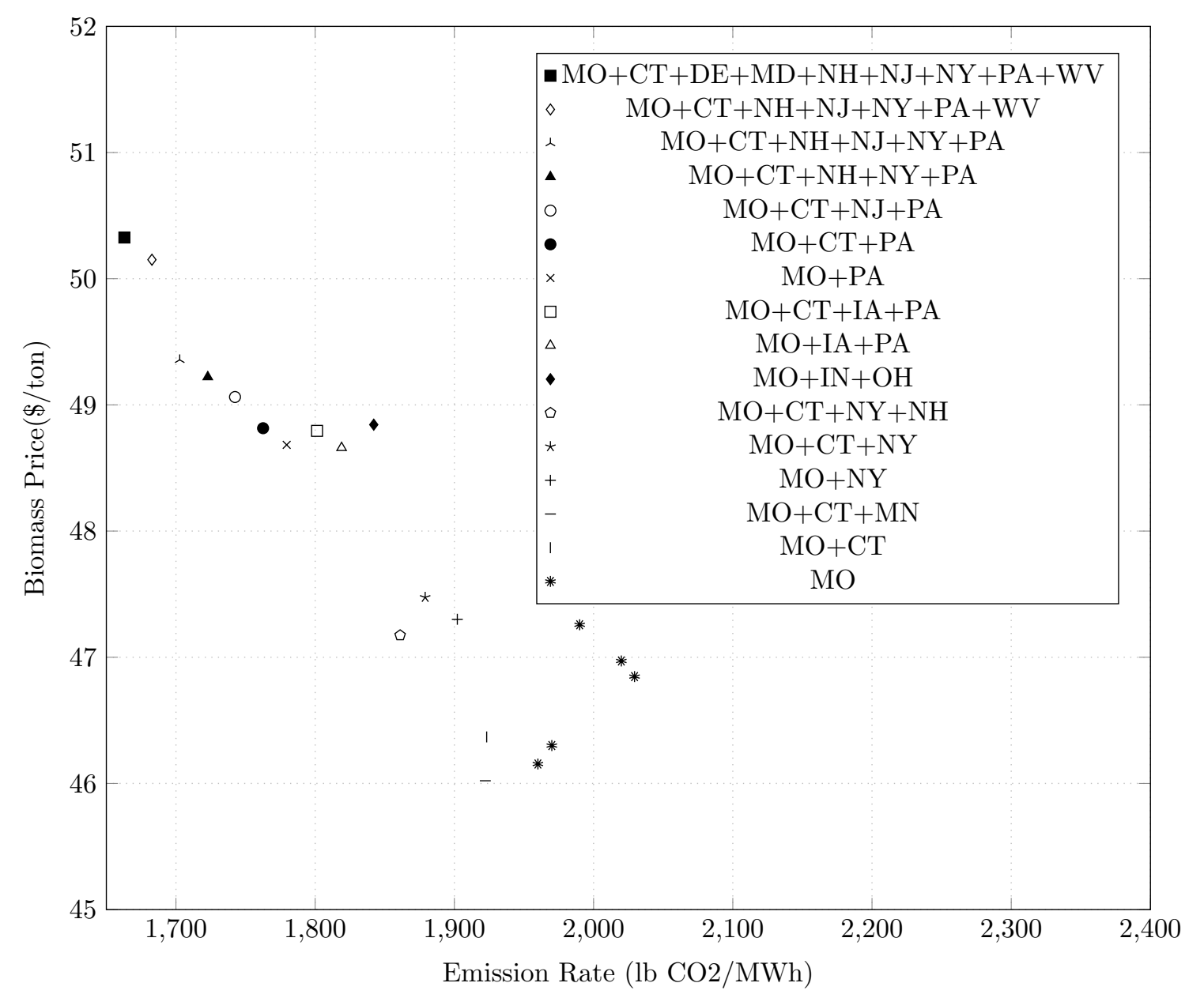

Figure 5.6: The impact of increased biomass demand on woody biomass price for nominal model,feasibility $\Psi=0 \%$

Figure 5.7 disaggregates these woody biomass prices into the woody biomass price for each state that enters into the multistate partnership for each solution having feasibility rate $\Psi=0 \%$. At the minimum achievable emission rate, the woody biomass price for the nine states that enter in the multistate plan varies across states. At 
that point, the highest woody biomass price is in WV with $\$ 52.23$ /ton, while the lowest woody biomass price is $\$ 46.32 /$ ton, in MO. Note, however, at that minimum achievable emission rate, WV contributes $115,463,515$ tons of woody biomass into the collaboration, while MO uses 19,885,553 tons of woody biomass. Note further that these state-level costs are themselves aggregations of many individual power plants, and some of the variability observed in a single state's costs is attributable to changes in decisions across plants.

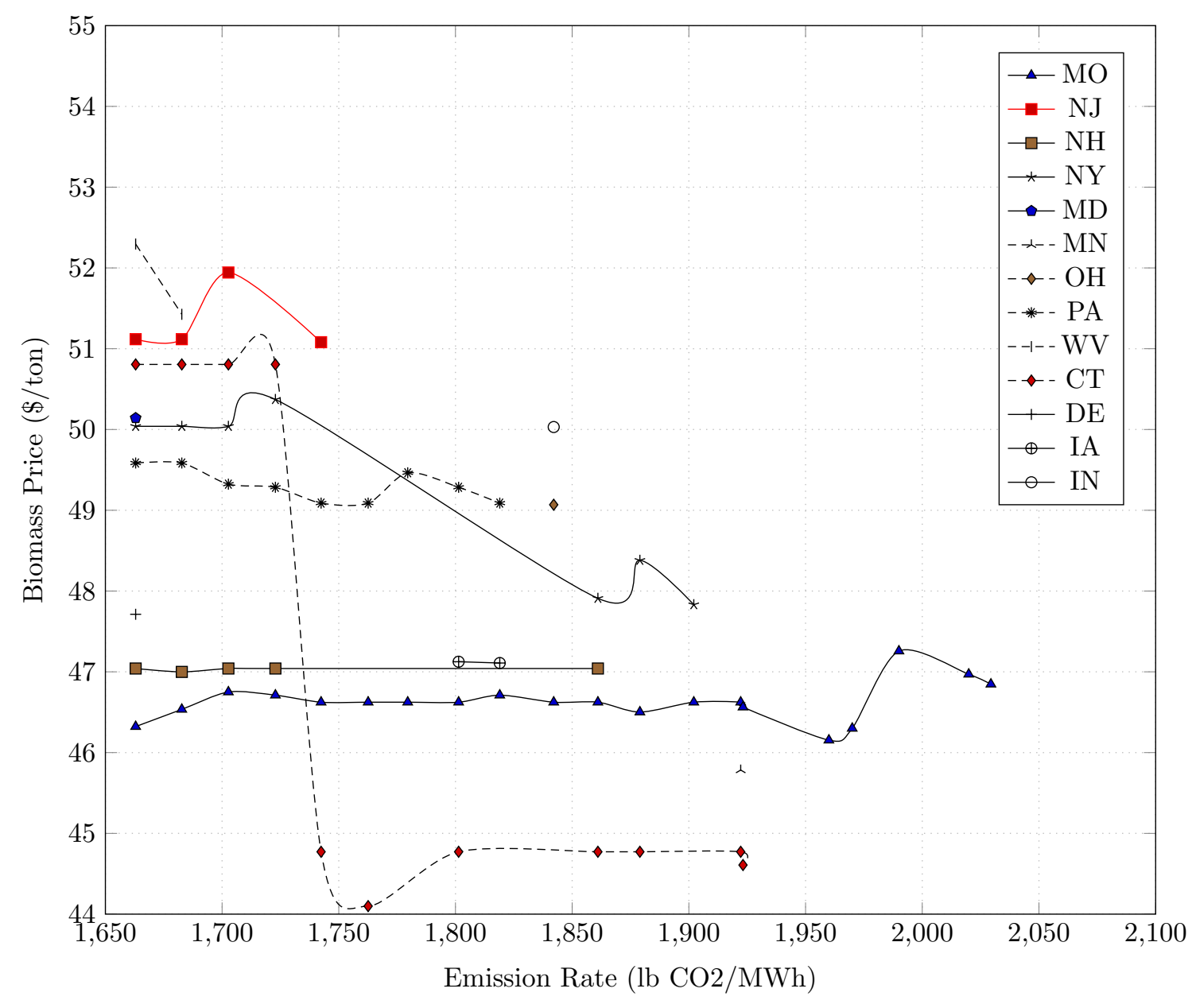

Figure 5.7: Detailed price of woody biomass for each state in the multi-state partnership based on nominal model, feasibility $\Psi=0 \%$ 
We also ran ROMCIA model for feasibility rate $\Psi=50 \%$ to observe how increased robustness impacts the multi-state plans' unit electricity generation cost and the minimum achievable emission rate. As demonstrated in Figure (5.8), it is apparent that the efficient frontier for feasibility $\Psi=50 \%$ consists of 11 distinct collaborations between emission rates of $1683 \mathrm{lb} / \mathrm{MWh}$ and $2050 \mathrm{lb} / \mathrm{MWh}$. Within those collaborations, MO partners most frequently with PA, entering into such partnerships in nine out of the 19 total points on the efficient frontier. This happens because PA has the highest potential woody biomass feedstock among the 18 states considered. Note further that, the minimum emission rate could be achieved is $1683 \mathrm{lb} / \mathrm{MWh}$, at which MO enters into a multi-state partnership with CT, MD, NJ, NY, PA, and WV. The unit electricity generation cost raises from $\$ 22.42$ / MWh to $\$ 40.08 / \mathrm{MWh}$ across this emission range of $1683 \mathrm{lb} / \mathrm{MWh}$ to $2050 \mathrm{lb} / \mathrm{MWh}$.

The change in woody biomass aggregated price per ton corresponding to the efficient frontier of feasibility $\Psi=50 \%$ is presented in Figure (5.9). Here, the same the general trend of aggregated woody biomass price increases as carbon emissions decrease can be observed, although as in Figure (5.7), there is considerable fluctuation around this general trend. At the minimum achievable emission rate, the woody biomass aggregated price is $\$ 50.41 /$ ton for the multi-state plan of MO, CT, MD, NJ, NY, PA, and WV. The lowest woody biomass aggregated price on the efficient frontier occurs at $1927 \mathrm{lb} / \mathrm{MWh}$ for the collaboration of MO,MN,and $\mathrm{OH}$.

Figure (5.10) disaggregates this into the woody biomass price for each state that enters into each multi-state plant on the efficient frontier for feasibility level $\Psi=50 \%$. As can be seen from the figure, at the minimum emission rate, the woody biomass price across the seven collaborating states varies between $\$ 51.82 /$ ton, in WV, and 


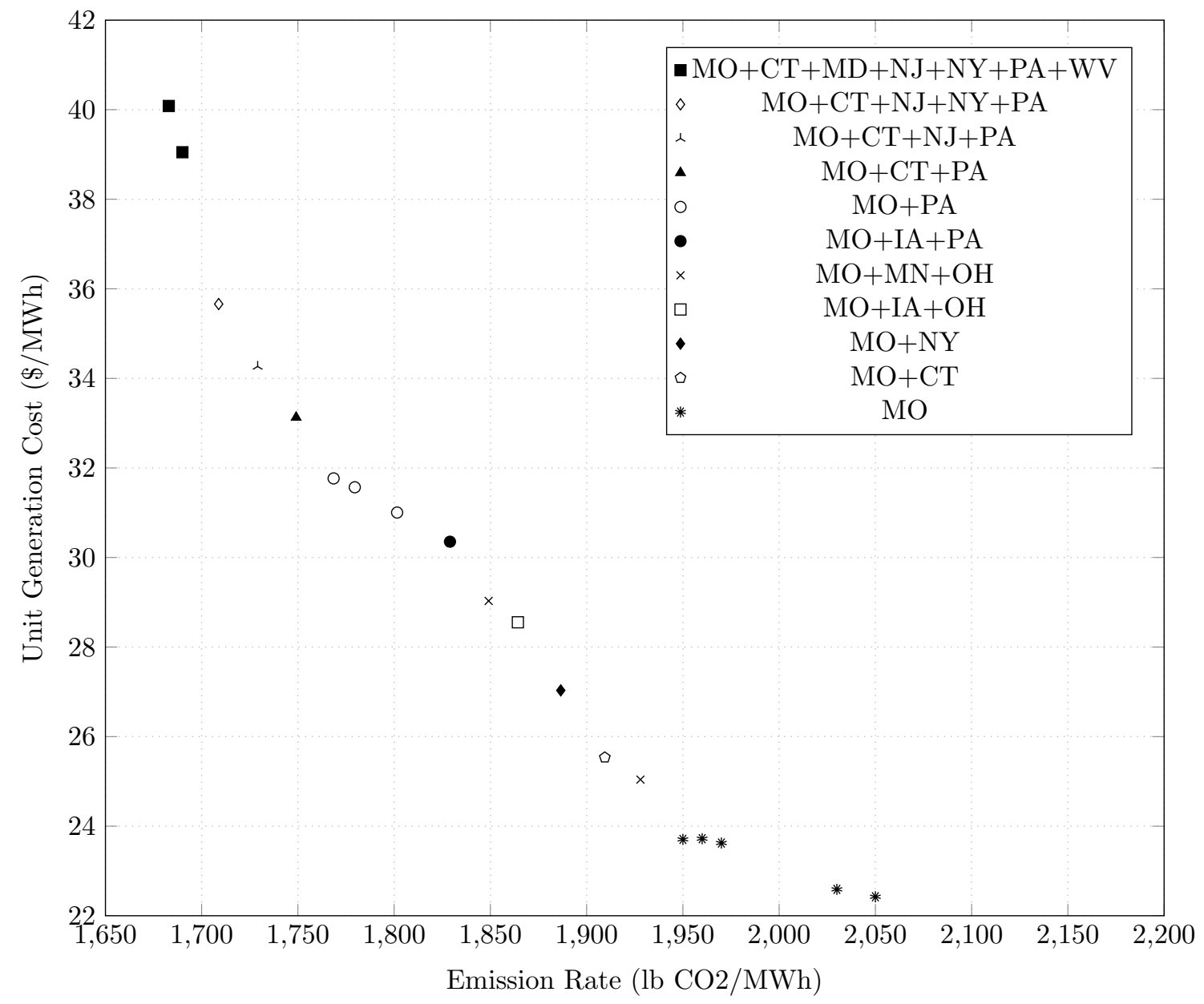

Figure 5.8: The efficient frontier of multi-state collaborations for feasibility $\Psi=50 \%$

$\$ 46.64$ /ton, in MO. Note, however, that the tons of woody biomass used in co-firing is $122,575,997$ and $11,205,793$ for $\mathrm{WV}$ and MO, respectively. Observe also in this figure that the woody biomass price for MO fluctuates very little between a minimum of $\$ 46 /$ ton and a maximum of $\$ 47 /$ ton across the range of emission rates for the emission $1682 \mathrm{lb} / \mathrm{MWh}$ to $2050 \mathrm{lb} / \mathrm{MWh}$.

The final set of runs performed using our ROMCIA examined the most robust feasibility scenario of $\Psi=90 \%$. Observe that there are ten separate collaborations on 


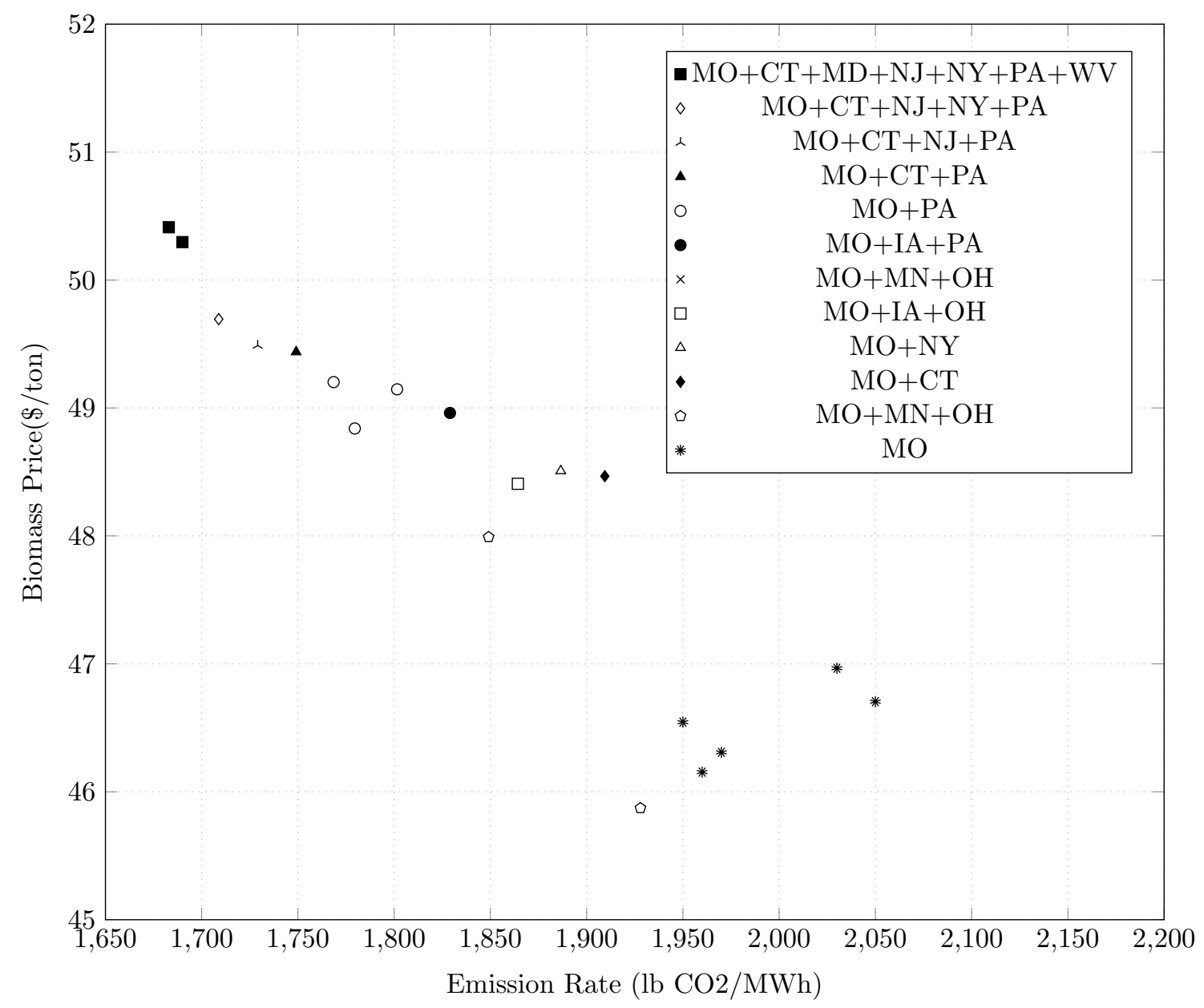

Figure 5.9: The impact of increased biomass demand on woody biomass price based on feasibility $\Psi=50 \%$

the efficient frontier for MO as seen in Figure (5.11). The unit electricity generation cost is between $\$ 39.79$ and $\$ 22.77$ for emission rates ranging from $1695 \mathrm{lb} / \mathrm{MWh}$ to $2070 \mathrm{lb} / \mathrm{MWh}$, respectively. At the minimum emission rate, MO enters into a partnership with MD, NJ, NY, PA, and WV. Here, the minimum efficient emission rate that can be achieved by MO when it operates independently is $1950 \mathrm{lb} / \mathrm{MWh}$ at unit electricity generation cost of $\$ 23.69$

As can be seen from Figure (5.12), the highest woody biomass aggregated price, 


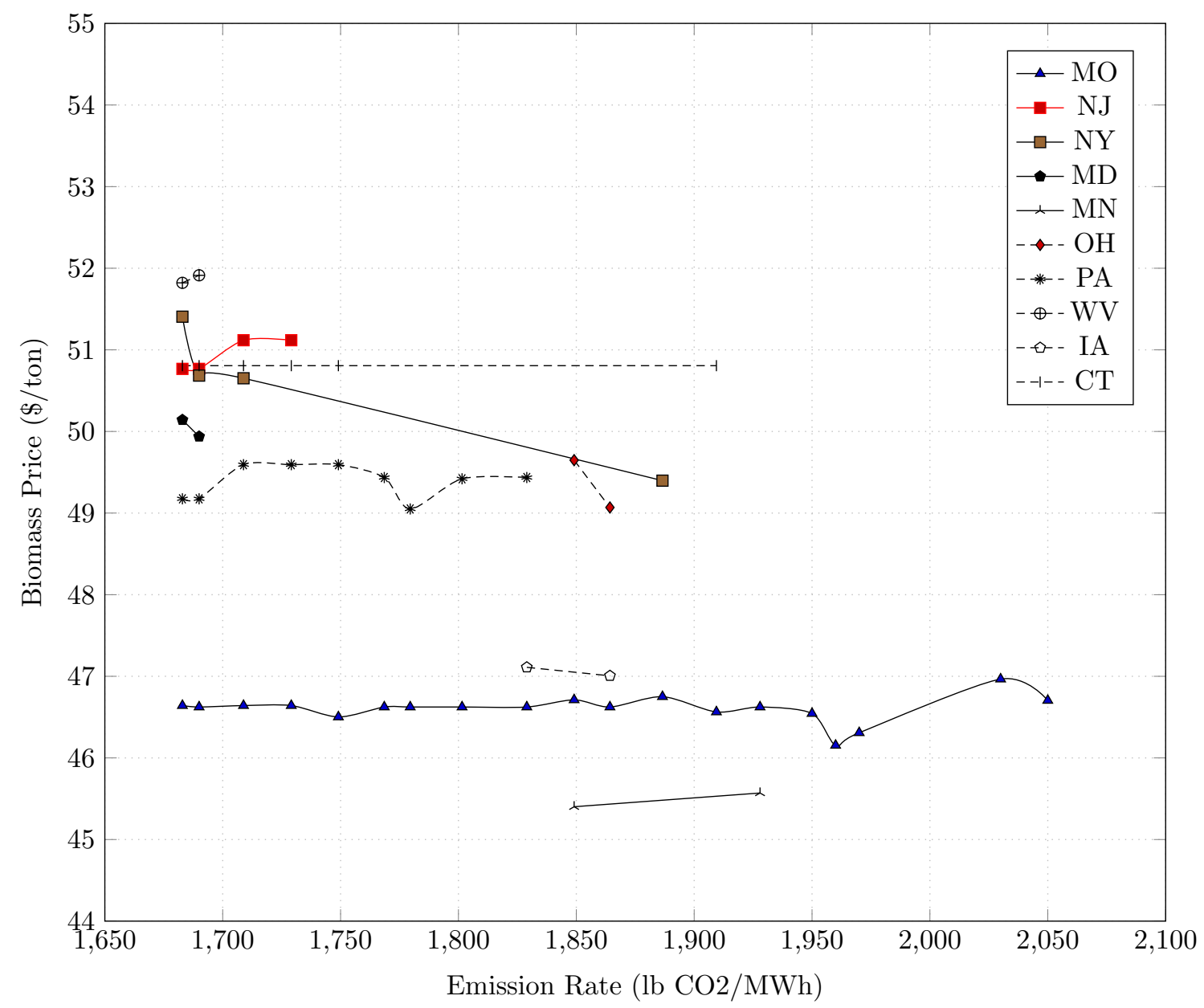

Figure 5.10: Detailed price of woody biomass for each state enter to a multi-state plan based on feasibility $\Psi=50 \%$

$\$ 50.50 /$ ton, occurs at the minimum emission rate of $1695 \mathrm{lb} / \mathrm{MWh}$. As discussed earlier, this increase in price is consistent with our expectations, due to the need for more woody biomass to achieve a lower emission rate. At the aggregated price of $\$ 50.50 /$ ton, a total $336,528,259$ ton of woody biomass is used in MD, NJ, NY, PA, and WV. Contrast this with the solution at emission rate $2070 \mathrm{lb} / \mathrm{MWh}$, which MO can achieve alone by utilizing 13,530,731 ton of woody biomass at a cost of $\$ 46.69$ /ton. 


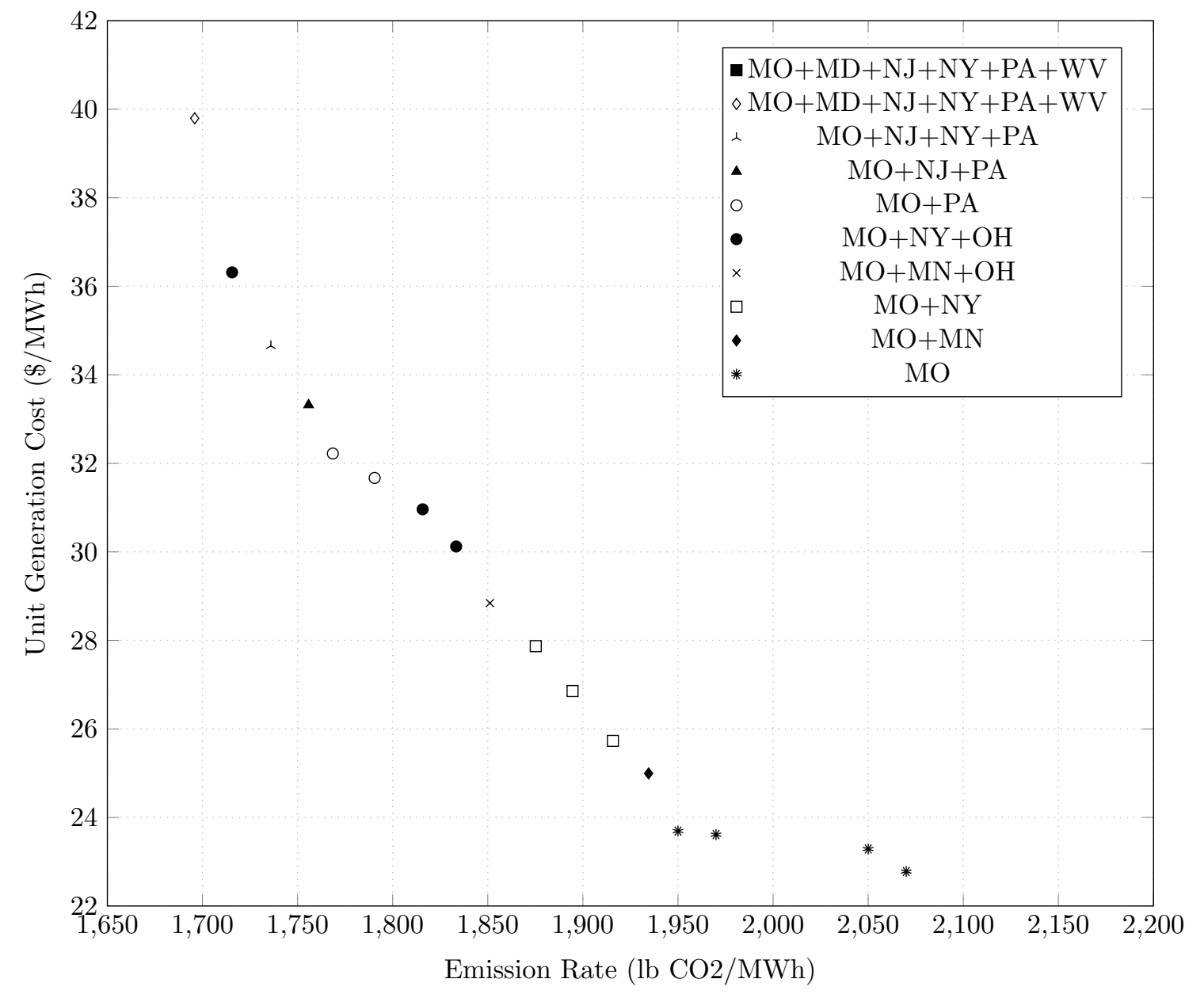

Figure 5.11: The efficient frontier of multi-state collaborations for feasibility $\Psi=$ $90 \%$

Figure (5.13) disaggregates this into the woody biomass price for each state that enters into the multi-state collaboration. As can be seen from the figure, at the minimum emission rate, the highest woody biomass price of $\$ 51.939$ /ton belongs to WV, while MO enters the partnership with the lowest price of $\$ 46.64 /$ ton. Note, however, that at that emission rate, WV burns $122,339,578$ tons of woody biomass in co-firing whereas MO cofires 11,205,793 tons of woody biomass.

Figure (5.14) illustrates the impact of the robustness level on the tradeoff between 


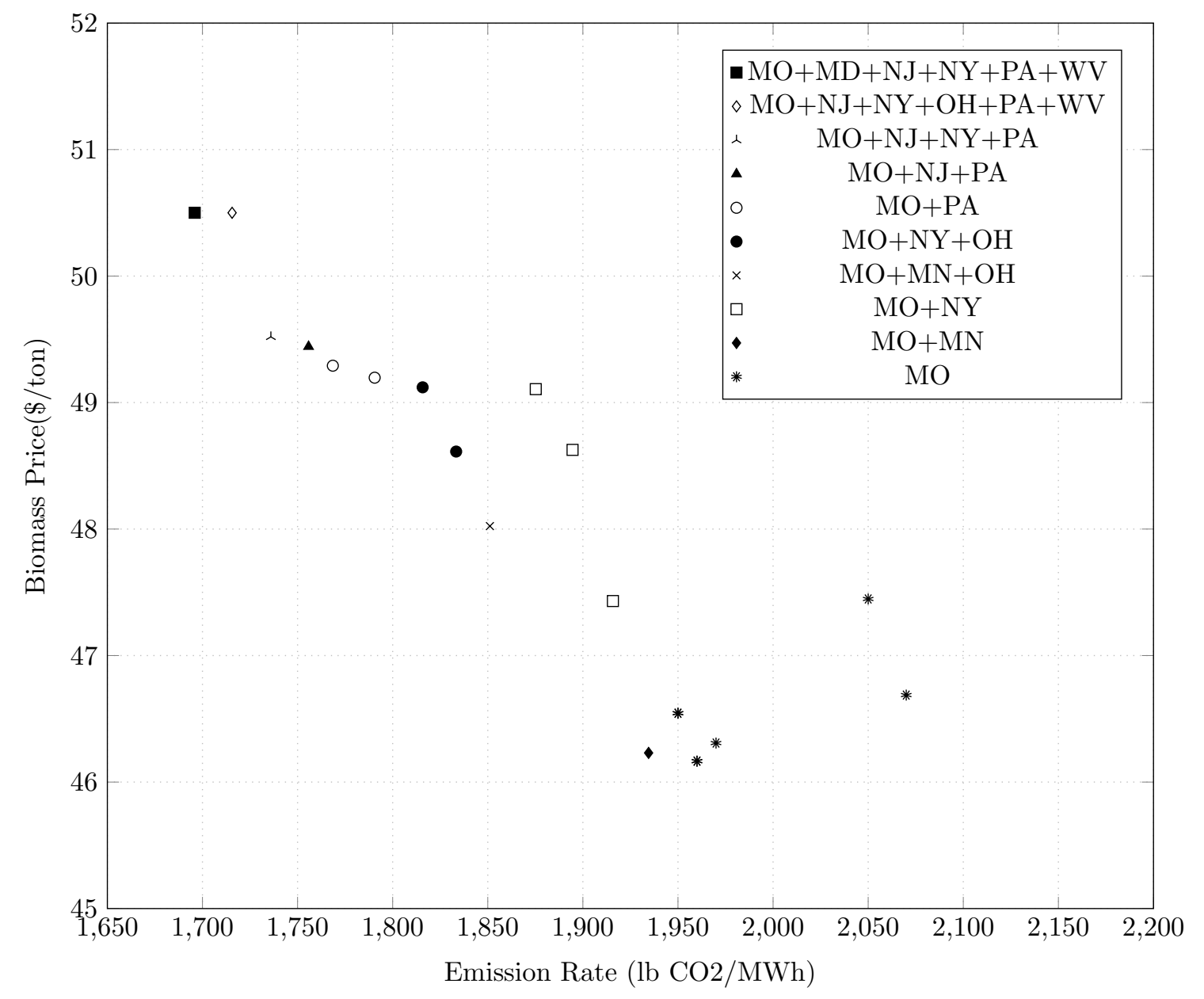

Figure 5.12: The impact of increased biomass demand on woody biomass price for feasibility $\Psi=90 \%$

the unit electricity generation cost and the minimum achievable emission rate. As can be seen from the figure, as the level of robustness increases, minimum-achievable emission rate also increases. Morever, at a fixed unit generation cost (say, for example, $\$ 40 / \mathrm{MWh}$ ), the minimum emission rate that MO can achieve through partnership increases as the robustness level increases (for this example, from $1662 \mathrm{lb} / \mathrm{MWh}$ for the non-robust scenario, to $1682 \mathrm{lb} / \mathrm{MWh}$ for feasibility scenario $\Psi=50 \%$ up to $1696 \mathrm{lb} / \mathrm{MWh}$ for feasibility level $\Psi=90 \%$. From a different perspective, the 


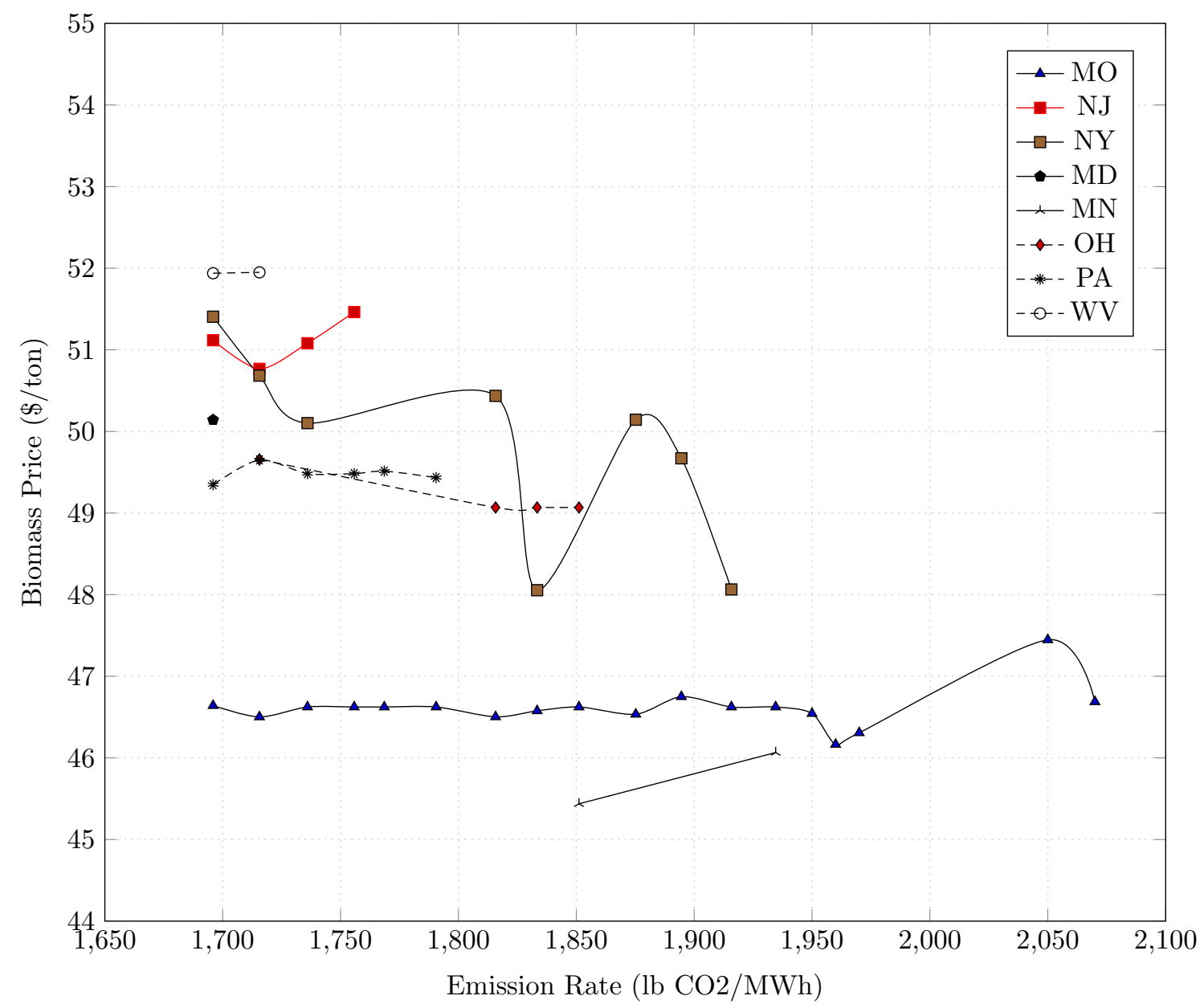

Figure 5.13: Detailed price of woody biomass for each state enter to a multi-state plan based on feasibility $\Psi=90 \%$

unit electricity generation cost increases to achieve a constant emission rate is more expensive when a higher rate of feasibility considered. For example, at an emission rate of $1700 \mathrm{lb} / \mathrm{MWh}$, for feasibility $\Psi=0 \%$ unit generation cost is $\$ 35.56$, whereas it is $\$ 37.24$ for feasibility $\Psi=50 \%$ and $\$ 39.07$ for the most robust case $\Psi=90 \%$. 


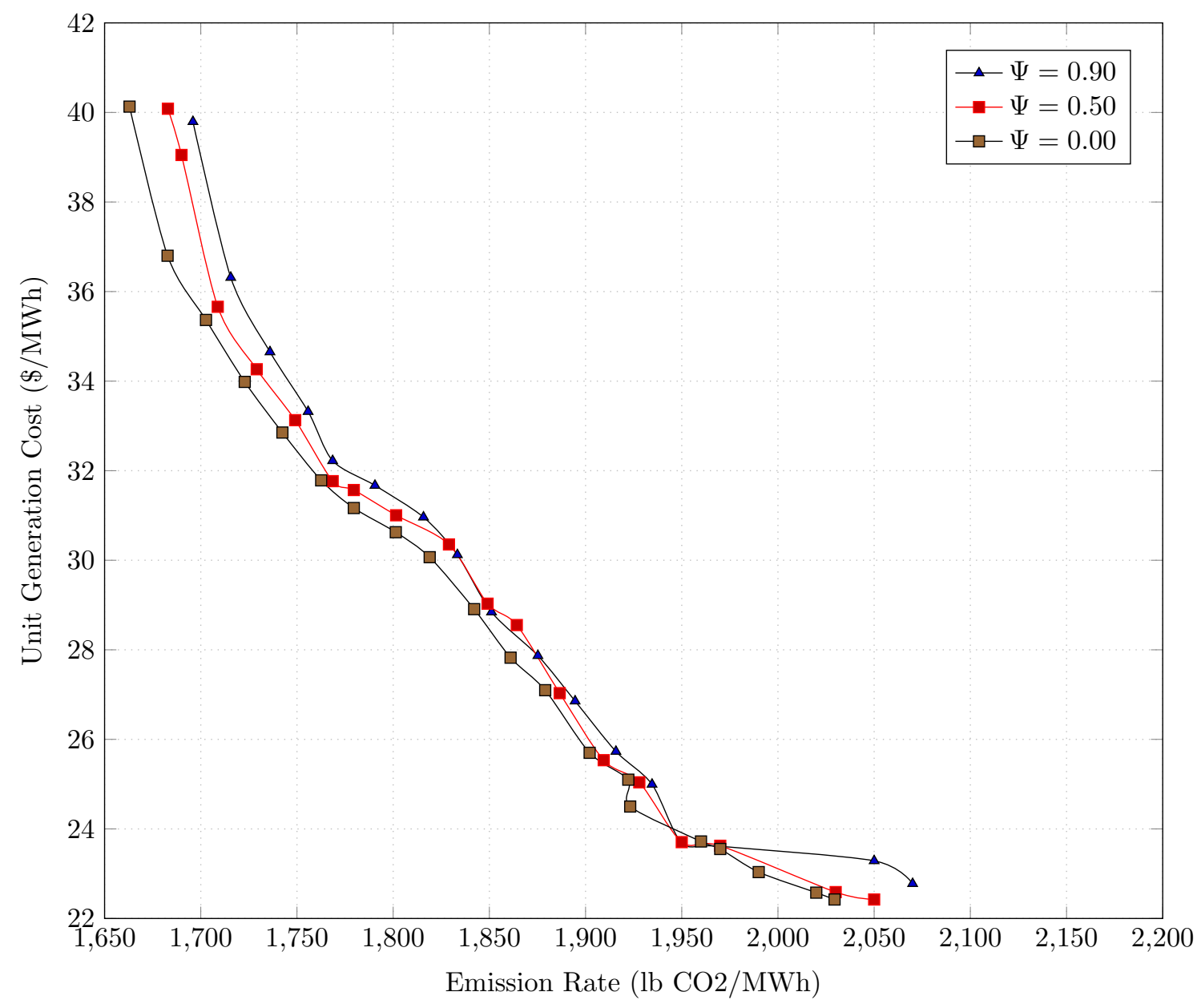

Figure 5.14: The impact of robustness on the emission rate and unit electricity generation cost 


\section{Chapter 6}

\section{Conclusions}

This chapter provides concluding remarks to this dissertation. In chapter 3, optimal multi-state collaborations were identified so as to reduce $\mathrm{CO} 2$ emissions by co-firing biomass in coal-burning power plants. In chapter 4, uncertainty in some parameters was addressed in these models identifying multi-state collaborations to reduce $\mathrm{CO} 2$ emissions. Finally, in chapter 5, a demand response model was included in the robust optimization model developed in the previous chapters in order to capture the relationship between demand and woody biomass price.

\subsection{Identifying Optimal Multi-state collaborations}

In chapter 3, an MILP model was developed to identify minimum-cost electricity generation approaches that reduce $\mathrm{CO}_{2}$ emissions through co-firing biomass across partnerships of multiple US states. A recent EPA proposal aims to cut carbon emissions from existing US power plants, providing each state with state-specific goals to 
mitigate carbon pollution from existing fossil fuel-fired EGUs, and allowing states the option to enter into multi-state partnerships that would cooperate to achieve aggregated goals. Because the MILP model was found to be computationally impractical, a marginal cost integrated approach (MCIA) model was developed to solve multi-state problems within short computational run times.

This modeling approach was utilized to determine optimal partnering strategies for one state, namely, Missouri among four potential partner states. Using the MCIA, we were able to identify an efficient frontier of solutions that achieve a specified $\mathrm{CO}_{2}$ emission level at minimum cost, with the optimal set of partners for Missouri changing as the allowable $\mathrm{CO}_{2}$ emission rate varies. None of the minimum-cost solutions obtained when Missouri operates independently lie on this efficient frontier, suggesting that multi-state partnerships are beneficial for Missouri at any $\mathrm{CO}_{2}$ emissions level. While many states are able to achieve their RPS targets for percentage of energy generated from renewable resources via co-firing biomass in existing coal-powered plants, co-firing biomass is not sufficient to achieve $\mathrm{EPA} \mathrm{CO}_{2}$ emission reduction targets on its own, although co-firing can achieve a sizeable contribution towards these emission reduction goals. States will need to consider other renewable energy sources along with the other three "building blocks" suggested by EPA (EPA 2014a) to reduce $\mathrm{CO}_{2}$ pollution to EPA's target levels. 


\subsection{Accounting for Uncertainty in Identifying Op- timal Multi-state collaborations}

Because there is significant uncertainty in many of the MILP model's data parameters, such as the facility upgrade costs necessary to utilize woody biomass in co-firing, in the this chapter we will extend the model to incorporate a robust optimization framework.

In chapter 4, an RO MILP model based on Bertsimas \& Sim (2003) was developed to identify minimum-cost multi-state partnerships for reducing $\mathrm{CO}_{2}$ emissions by co-firing woody biomass with coal. This model specifically accounts for uncertainty in three key input data parameters: the coal electricity generation cost, the boiler upgrade cost necessary to allow for a biomass-specific boiler, and the $\mathrm{CO}_{2}$ emission rates of power plants. Because the RO MILP model was not able to solve large instances within a reasonable computational run time, the RO MILP was used to generate a computationally-efficient robust marginal cost integrated approach (ROMCIA) optimization model. This ROMCIA can identify the efficient frontier of potential partnerships achieving minimum-cost solutions across a range of potential emissions levels. The ROMCIA model further allows the user to specify a required probability of achieving the desired emissions level, accounting for the assumed uncertainty in power plant emission levels.

The analytic approach was demonstrated with application to a set of 18 Northern US states, identifying an efficient frontier of minimum-cost partnerships that one state (Missouri) might enter into, based on the desired emissions level and probability of feasibility. These ROMCIA solutions show that the optimal set of partners for Missouri changes as the allowable $\mathrm{CO}_{2}$ emission rate changes. Moreover, as the desired probability of feasibility increases, the analysis demonstrates the correspond- 
ing increase in the electricity generation cost, presenting the "price of robustness" to decision makers.

\subsection{A Demand Response Model for Biopower Gen- eration to Identify Optimal Multi-State Col- laborations}

Note that the demand for biomass in previous models is not generated based on cost efficiencies relative to coal, instead this demand occurs due to the desire to reduce emission levels. It is reasonable to expect that, as demand for woody biomass feedstock increases, the procurement cost would increase.

In chapter 5, a Demand Response Model (DRM) was developed and incorporated in into an MINLP model, extending the robust optimization model developed in chapter 4. This DRM shows the economic relationship between woody biomass price and a policy forced demand. The $\beta$ parameter, denoting the elasticity of demand, was estimated based on historical data of woody biomass consumption and price, and was assumed to apply to all states. As discussed in section (5.1), a base parameter $\alpha_{p r}^{t}$, was computed separately for each plant considered in this study. Our robust MINLP was solved with a two-stage approach. Moreover, grid computing using the high-performance computing infrastructure provided by Research Computing Support Services of the University of Missouri was utilized to generate the single state RMCTs, which significantly reduced the computational time.

Based on the robust MINLP incorporating a DRM, the power plant-based woody biomass price was seen to be significantly affected by increased demand. As expected, 
the aggregated woody biomass price is higher at lower emission rates. That is because the states that have significant woody biomass resources in these collaborations also have higher woody biomass prices. Our robust MINLP computational results suggest that, operating independently, MO can efficiently achieve emission rates of 1950 lb/MWh and higher. However, in order to achieve any emission rate less than 1950 lb/MWh, it is advantageous from a cost perspective for MO to enter into multistate partnerships. If uncertainty in emission rates is not considered (i.e., if we use the non-robust model, $\Psi=0 \%$, MO can achieve a $14.7 \%$ emission reduction by partnering with other states compared to the $1950 \mathrm{lb} / \mathrm{MWh}$ that MO achieve alone. If uncertainty in data parameters is included in the model, MO can achieve $13.7 \%$ and $13 \%$ emission reductions below this $1950 \mathrm{lb} / \mathrm{MWh}$ level by partnering with other states, corresponding to feasibility levels $\Psi=50 \%$ and $\Psi=90 \%$, respectively.

\subsection{Future works}

We developed the robust MINLP model to identify optimal partnerships for MO across a range of emission rates and feasibility probabilities of achieving those emission rates. In these models we took into account both economic and environmental perspectives related to questions about generating biopower from woody biomass cofiring with coal. One could extend this study by considering also the social and public policy aspects of developing optimal multi-state collaborations. Moreover, as discussed earlier, we considered only woody biomass as a renewable energy resource to satisfy EPA and states RPS environmental targets. Other types of renewable resources such wind, and solar could be added into the developed models to determine 
the total renewable potential of each state.

Based on data provided from (EIA 2016b), in 2016, the share of electricity generated from natural gas for the first time exceeded the share of electricity generated from coal. Note that natural gas emits less carbon than does coal (EIA 2017a). Thus, in future work, natural gas could also be included in our optimization models along with coal and woody biomass as a fuel source to generate electricity at lower emission levels.

As a limitation of this research, we assumed that there are no transaction costs between collaborating states. Essentially, we assumed that all parties participating in multistate collaborations are meeting their obligations in the contract and that the transaction would be frictionless. Therefore, this study could be extended by considering the transaction cost of carbon trading between partners so as to maximize overall benefit of partnerships for the mandatory policies.

The price elasticity of demand was based on a ten year period due to the available data. However, it is possible that over a longer term, the increased prices following increased demand would lead to increased woody biomass production. It is possible that with a different data set in the future, one might be able to develop a more dynamic model that operates over the woody biomass production lifecycle.

Finally, in our models, we considered three radii $(30 \mathrm{~km}, 60 \mathrm{~km}$, and $90 \mathrm{~km})$ to procure woody biomass for co-firing. However, if the woody biomass price was sufficiently high in the future, densification options (e.g., pellets) for woody biomass could become economically competitive, allowing for an increased radius from which the woody biomass is sourced. That, in turn, might make it possible to supply more woody biomass to power plants for co-firing, which might increase the potential 
biopower generation of states. 


\section{Bibliography}

AEO (2013), 'Annual Energy Outlook, with Projections to 2040 ,institution=DOE/EIA-0383, USA'.

Aguilar, F. X. (2014), Wood energy in developed economies: resource management, economics and policy, Routledge.

Aguilar, F. X. \& Garrett, H. E. (2009), 'Perspectives of Woody Biomass for Energy: Survey of State Foresters, State Energy Biomass Contacts, and National Council of Forestry Association Executives', Journal of Forestry 107(6), 297-306.

Aguilar, F. X., Goerndt, M. E., Song, N. \& Shifley, S. (2012), 'Internal, external and location factors influencing cofiring of biomass with coal in the US northern region', Energy Economics 34(6), 1790-1798.

Aguilar, F. X. \& Saunders, A. (2010), 'Policy instruments promoting wood-to-energy uses in the continental United States', Journal of Forestry 108(3), 132-140.

Alam, M. B., Pulkki, R., Shahi, C. \& Upadhyay, T. (2012), 'Modeling woody biomass procurement for bioenergy production at the Atikokan generating station in northwestern Ontario, Canada', Energies 5(12), 5065-5085. 
Alam, M., Pulkki, R., Shahi, C. et al. (2012), 'Road network optimization model for supplying woody biomass feedstock for energy production in northwestern Ontario', The open forest science Journal 5, 1-14.

Ben-Tal, A. \& Nemirovski, A. (1999), 'Robust solutions of uncertain linear programs', Operations research letters 25(1), 1-13.

Bentsen, N. S., Jack, M. W., Felby, C. \& Thorsen, B. J. (2014), 'Allocation of biomass resources for minimising energy system greenhouse gas emissions', Energy 69, 506515.

Bertsimas, D. \& Sim, M. (2003), 'Robust discrete optimization and network flows', Mathematical programming 98(1-3), 49-71.

Bertsimas, D. \& Sim, M. (2004), 'The price of robustness', Operations research 52(1), 35-53.

Bigerna, S., Bollino, C. A. \& Micheli, S. (2016), 'Costs assessments of European environmental policies', Computers \& Operations Research 66, 327-335.

Boden, T., Marland, G. \& Andres, R. (2015), 'National co2 emissions from fossil-fuel burning, cement manufacture, and gas flaring: 1751-2007. carbon dioxide information analysis center oak ridge national laboratory'.

Bussieck, M. R., Ferris, M. C. \& Meeraus, A. (2009), 'Grid-enabled optimization with gams', INFORMS Journal on Computing 21(3), 349-362.

Calfa, B. A. \& Grossmann, I. E. (2015), 'Optimal procurement contract selection with price optimization under uncertainty for process networks', Computers $\& 3$ Chemical Engineering 82, 330-343. 
Cambero, C. \& Sowlati, T. (2014), 'Assessment and optimization of forest biomass supply chains from economic, social and environmental perspectives-a review of literature', Renewable and Sustainable Energy Reviews 36, 62-73.

Chen, Y.-H., Lu, S.-Y., Chang, Y.-R., Lee, T.-T. \& Hu, M.-C. (2013), 'Economic analysis and optimal energy management models for microgrid systems: A case study in Taiwan', Applied Energy 103, 145-154.

Daigneault, A., Sohngen, B. \& Sedjo, R. (2012), 'Economic approach to assess the forest carbon implications of biomass energy', Environmental Science $\&$ Technology 46(11), 5664-5671.

Dale, V. H., Kline, K. L., Marland, G. \& Miner, R. A. (2015), 'Ecological objectives can be achieved with wood-derived bioenergy', Frontiers in Ecology and the Environment 13, 297-299.

DOE (2013), 'U.S. Departmant of Energy, Renewable Energy Data Book'.

URL: http://www.nrel.gov/docs/fy15osti/62580.pdf

Douglas, I., Alam, K., Maghenda, M., Mcdonnell, Y., McLean, L. \& Campbell, J. (2008), 'Unjust waters: climate change, flooding and the urban poor in Africa', Environment and Urbanization 20(1), 187-205.

Driscoll, C. T., Buonocore, J. J., Levy, J. I., Lambert, K. F., Burtraw, D., Reid, S. B., Fakhraei, H. \& Schwartz, J. (2015), 'Us power plant carbon standards and clean air and health co-benefits', Nature Climate Change 5(6), 535-540.

DSIRE (2016), 'Database of State Incentives for Renewables and Efficiency', http://programs.dsireusa.org/system/program/maps. 
Du, X. \& Runge, T. (2014), 'Price dynamics in wisconsin woody biomass markets', Biomass and Bioenergy 63, 250-256.

Ebers, A., Malmsheimer, R. W., Volk, T. A. \& Newman, D. H. (2016), 'Inventory and classification of United States federal and state forest biomass electricity and heat policies', Biomass and Bioenergy 84, 67-75.

EIA (2014a), 'U.S. Energy Information Administration, Annual Energy Outlook 2014,DOE/EIA-0383, USA'.

EIA (2014b), 'U.S. Energy Information Administration, State Energy Data 2014: Prices and Expenditures'.

EIA (2016a), 'Frequently Asked Questions: How much coal, natural gas, or petroleum is used to generate a kilowatthour of electricity'.

URL: http://www.eia.gov/tools/faq/faq.cfm? $i d=667 / 84 t=2$

EIA (2016b), 'U.S. Energy Information Administration, Electcity Net Generation in 2016', https://www.eia.gov/electricity/data/browser.

EIA (2017a), 'U.S. Energy Information Administration', https://www.eia.gov/ tools $/$ faqs $/$ faq.php?id=73\&t $=11$.

EIA (2017b), 'U.S. Energy Information Administration, February 2017 Monthly Energy Review, Renewable Energy Consumption: Electric Power Sector'.

EIA, U. (2013), 'Annual energy outlook 2013', US Energy Information Administration, Washington, DC pp. 60-62. 
Ekşioğlu, S. D., Acharya, A., Leightley, L. E. \& Arora, S. (2009), 'Analyzing the design and management of biomass-to-biorefinery supply chain', Computers $\&$ Industrial Engineering 57(4), 1342-1352.

EPA (2014a), 'Environmental Protection Agency 40 CFR Part 62, volume=79, no $=5384^{\prime}$.

EPA (2014b), 'Summary Tables eGRID 9th edition Version 1.02014 '.

EPA (2015), 'Environmental Protection Agency 40 CFR Part 60; Carbon Pollution Emission Guidelines for Existing Stationary, volume=RIN 2060-AR33, no=5384'.

EPA (2016), 'Inventory of US greenhouse gas emissions and sinks: 1990-2014'.

EU, A. (2014), 'A policy framework for climate and energy in the period from 2020 to 2030 '.

European Commission (2016), '2030 Energy Stratgey'.

URL: http://ec.europa.eu/energy/en/topics/energy-strategy/2030-energy-strategy

Foo, D. C., Tan, R. R., Lam, H. L., Aziz, M. K. A. \& Klemeš, J. J. (2013), 'Robust models for the synthesis of flexible palm oil-based regional bioenergy supply chain', Energy 55, 68-73.

Frombo, F., Minciardi, R., Robba, M., Rosso, F. \& Sacile, R. (2009), 'Planning woody biomass logistics for energy production: A strategic decision model', Biomass and Bioenergy 33(3), 372-383.

Galik, C. S., Abt, R. \& Wu, Y. (2009), 'Forest biomass supply in the southeastern 
united states - implications for industrial roundwood and bioenergy production', Journal of Forestry 107(2), 69-77.

GAMS (2017), 'GAMS Development Corporation, General Algebraic Modeling System (GAMS) Release 24.9.1. Washington, DC, USA'.

URL: http://www.gams.com

Georgiou, P. N. (2016), 'A bottom-up optimization model for the long-term energy planning of the Greek power supply sector integrating mainland and insular electric systems', Computers \& Operations Research 66, 292-312.

Goerndt, M. E., Aguilar, F. X., Miles, P., Shifley, S., Song, N. \& Stelzer, H. (2012), 'Regional assessment of woody biomass physical availability as an energy feedstock for combined combustion in the US northern region', Journal of Forestry 110(3), 138-148.

Goerndt, M. E., Aguilar, F. X. \& Skog, K. (2013), 'Resource potential for renewable energy generation from co-firing of woody biomass with coal in the Northern U.S. ', Biomass and Bioenergy 59(0), $348-361$.

Gómez-Calvet, R., Conesa, D., Gómez-Calvet, A. R. \& Tortosa-Ausina, E. (2016), 'On the dynamics of eco-efficiency performance in the European Union', Computers E3 Operations Research 66, 336-350.

Gong, J., Garcia, D. J. \& You, F. (2016), 'Unraveling optimal biomass processing routes from bioconversion product and process networks under uncertainty: An adaptive robust optimization approach', ACS Sustainable Chemistry 83 Engineering 4(6), 3160-3173. 
Gurobi Optimization (2016), 'Gurobi Optimizer Reference Manual'.

URL: http://www.gurobi.com

Hashim, H., Douglas, P., Elkamel, A. \& Croiset, E. (2005), 'Optimization model for energy planning with $\mathrm{CO} 2$ emission considerations', Industrial \&3 Engineering Chemistry Research 44(4), 879-890.

Hu, M.-C., Lin, C.-H., Chou, C.-A., Hsu, S.-Y. \& Wen, T.-H. (2011), 'Analysis of biomass co-firing systems in Taiwan power markets using linear complementarity models', Energy Policy 39(8), 4594-4600.

Huang, J., Leng, M. \& Parlar, M. (2013), 'Demand functions in decision modeling: A comprehensive survey and research directions', Decision Sciences 44(3), 557-609.

Jiang, R., Wang, J. \& Guan, Y. (2012), 'Robust unit commitment with wind power and pumped storage hydro', IEEE Transactions on Power Systems 27(2), 800-810.

Kanudia, A. \& Shukla, P. (1998), 'Modelling of uncertainties and price elastic demands in energy-environment planning for india', Omega 26(3), 409-423.

Kaplan, U., Türkay, M., Karasözen, B. \& Biegler, L. T. (2011), 'Optimization of supply chain systems with price elasticity of demand', INFORMS Journal on Computing 23(4), 557-568.

Karl, T. R. (2009), Global climate change impacts in the United States, Cambridge University Press.

Kim, J., Realff, M. J., Lee, J. H., Whittaker, C. \& Furtner, L. (2011), 'Design of biomass processing network for biofuel production using an MILP model', Biomass and Bioenergy 35(2), 853-871. 
Kintisch, E. (2016), 'New solution to carbon pollution?', Science 352(6291), $1262-$ 1263.

Koo, J., Han, K. \& Yoon, E. S. (2011), 'Integration of ccs, emissions trading and volatilities of fuel prices into sustainable energy planning, and its robust optimization', Renewable and Sustainable Energy Reviews 15(1), 665-672.

Lauri, P., Havlík, P., Kindermann, G., Forsell, N., Böttcher, H. \& Obersteiner, M. (2014), 'Woody biomass energy potential in 2050', Energy Policy 66, 19-31.

Liu, Z., Altman, I. \& Johnson, T. G. (2014), 'The feasibility of co-firing biomass for electricity in Missouri', Biomass and Bioenergy 69, 12-20.

Miner, R. A., Abt, R. C., Bowyer, J. L., Buford, M. A., Malmsheimer, R. W., O’Laughlin, J., Oneil, E. E., Sedjo, R. A. \& Skog, K. E. (2014), 'Forest Carbon Accounting Considerations in US Bioenergy Policy', Journal of Forestry 112(6), 591606.

Misener, R. \& Floudas, C. A. (2014), 'Antigone: algorithms for continuous/integer global optimization of nonlinear equations', Journal of Global Optimization 59(23), 503-526.

Mobini, M., Sowlati, T. \& Sokhansanj, S. (2011), 'Forest biomass supply logistics for a power plant using the discrete-event simulation approach', Applied Energy 88(4), 1241-1250.

Moret, S., Bierlaire, M. \& Maréchal, F. (2014), Robust optimization for strategic energy planning, Technical report, Transport and Mobility Laboratory. 
Nytimes (2017a), 'E.P.A. Announces Repeal of Major Obama-Era Carbon Emissions Rule', https://www.nytimes.com/2017/10/09/climate/clean-power-plan. html?_r=0.

Nytimes (2017b), 'How Can U.S. States Fight Climate Change if Trump Quits the Paris Accord?', https://www.nytimes.com/2017/09/20/climate/ paris-climate-accord-trump.html.

Omu, A., Choudhary, R. \& Boies, A. (2013), 'Distributed energy resource system optimisation using mixed integer linear programming', Energy Policy 61, 249-266.

Parisio, A., Del Vecchio, C. \& Vaccaro, A. (2012), 'A robust optimization approach to energy hub management', International Journal of Electrical Power $\mathcal{E}$ Energy Systems 42(1), 98-104.

Python Software Foundation (2016), 'Python Programming Language'. URL: /http://www.python.org/

Rentizelas, A., Tatsiopoulos, I. \& Tolis, A. (2009), 'An optimization model for multibiomass tri-generation energy supply', Biomass and Bioenergy 33(2), 223-233.

Shabani, N. \& Sowlati, T. (2013), 'A mixed integer non-linear programming model for tactical value chain optimization of a wood biomass power plant', Applied Energy 104, 353-361.

Shabani, N. \& Sowlati, T. (2016), 'A hybrid multi-stage stochastic programmingrobust optimization model for maximizing the supply chain of a forest-based biomass power plant considering uncertainties', Journal of Cleaner Production 112, 3285-3293. 
Solomon, S., Plattner, G.-K., Knutti, R. \& Friedlingstein, P. (2009), 'Irreversible climate change due to carbon dioxide emissions', Proceedings of the National Academy of Sciences pp. pnas-0812721106.

Song, H., Dotzauer, E., Thorin, E., Guziana, B., Huopana, T. \& Yan, J. (2012), 'A dynamic model to optimize a regional energy system with waste and crops as energy resources for greenhouse gases mitigation', Energy 46(1), 522-532.

Soyster, A. L. (1973), 'Technical note - convex programming with set-inclusive constraints and applications to inexact linear programming', Operations research $21(5), 1154-1157$.

Tay, D. H., Ng, D. K. \& Tan, R. R. (2013), 'Robust optimization approach for synthesis of integrated biorefineries with supply and demand uncertainties', Environmental Progress \& Sustainable Energy 32(2), 384-389.

Tidball, R., Bluestein, J., Rodriguez, N. \& Knoke, S. (2010), 'Cost and performance assumptions for modeling electricity generation technologies', Contract 303, 275300.

Urwin, K. \& Jordan, A. (2008), 'Does public policy support or undermine climate change adaptation? Exploring policy interplay across different scales of governance', Global Environmental Change 18(1), 180-191.

Van Dyken, S., Bakken, B. H. \& Skjelbred, H. I. (2010), 'Linear mixed-integer models for biomass supply chains with transport, storage and processing', Energy 35(3), 1338-1350. 
Wang, R., Wang, P. \& Xiao, G. (2015), 'A robust optimization approach for energy generation scheduling in microgrids', Energy Conversion and Management 106, 597-607.

Zhang, Y., Hu, G. \& Brown, R. C. (2014), 'Integrated supply chain design for commodity chemicals production via woody biomass fast pyrolysis and upgrading', Bioresource Technology 157, 28-36.

Zhu, X. \& Yao, Q. (2011), 'Logistics system design for biomass-to-bioenergy industry with multiple types of feedstocks', Bioresource Technology 102(23), 10936-10945.

Zografidou, E., Petridis, K., Arabatzis, G. \& Dey, P. K. (2016), 'Optimal design of the renewable energy map of Greece using weighted goal-programming and data envelopment analysis', Computers \&3 Operations Research 66, 313-326. 


\section{VITA}

Bayram Dundar was born in Malatya, Turkey. He completed his Ph.D. in Industrial and Manufacturing Systems Engineering at the University of Missouri under the advisement of Dr. Ronald McGarvey. He also earned his M.S. degree in Industrial Engineering from the University of Missouri in 2013 and received a Bachelor Degree from the Selcuk University, Turkey in 2008. His Ph.D. research involved applying robust optimization techniques to energy policy problems, but he has also gained experience applying such mathematical models other sustainability problems. His research interests center on applying optimization techniques to healthcare, energy, and sustainability-related problems in the presence of uncertainty. Additionally, he has industry experience as a production planning engineer. He has published research in leading journals such as Computers $\mathcal{E}$ Industrial Engineering, Journal of Biomedical Informatics, and Environment Systems $\&$ Decisions. Bayram is a member of the Institute for Operations Research and the Management Sciences (INFORMS) and the Institute of Industrial and Systems Engineers (IISE). 